\title{
Synchronous Amplification of Subpicosecond Pulses
}

\author{
by \\ James David Kafka
Submitted in Partial Fulfillment of the
Requirements for the Degree
DOCTOR OF PHILOSOPHY

Supervised by Professors Conger W. Gabel and Gerard Mourou The Institute of Optics

\author{
University of Rochester \\ Rochester, New York
}


James David Kafka was born in New York, New York in 1956. He entered the University of Rochester in 1973 and began study at the Institute of optics. In 1977 he received the B. S. degree in Optics with distinction.

In 1977, he began graduate studies at the Institute of Optics. During much of his residence, he has studied under Professors Conger Gabel and Gerard Mourou, who have also supervised his thesis research. Mr. Kafka has been a member of the Picosecond group at the Institute of Optics and the Laboratory for Laser Energetics, University of Rochester. As such, he has pursued research into picosecond pulses and dye laser amplifiers, the subject of this dissertation. While at the Laboratory for Laser Energetics, he participated in the construction of the subpicosecond Biological Physics Facility.

Mr. Kafka was a recipient of the Institute Fellowship in 1977 and the Xerox Fellowship the following year. From 1979 through 1983, he was a Laser Energetics Fellow. He is a member of the Optical Society of America. 


\section{ACKNOWLEDGEMENTS}

I am deeply grateful to my parents for their support during the course of this thesis work. Their love and encouragement were a constant source of comfort to me.

I am also pleased to acknowledge my advisors: Dr. Conger Gabel and Dr. Gerard Mourou. Conger has supplied invaluable advice, of both a scientific and personal nature. His expert guidance was a key element in the excecution and preparation of the thesis. Gerard, whose ability to generate new ideas is unsurpassed, has contributed greatly to my education as a scientist. Both advisors have left a clear mark of their participation on this thesis.

Many of the experiments in this thesis would not have been possible without the work of Tod Sizer and Irl Duling. Tod created the oscillator system and Irl the RF coupled system used in my investigations. In addition, I wish to acknowledge their participation and support in all phases of my research.

I am indebted to the Institute of optics and the Laboratory for Laser Energetics, both financially, and for providing a stimulating atmosphere for learning. I wish to 
thank the faculty and staff of these institutions and in particular Mike Raymer for guidance and support. I also wish to thank Peter Drummond and steve Craxton for their time and assistance with the theoretical portion of this thesis.

Finally, I wish to thank all of the opticists, volleyball players and musicians who have made living and growing up in Rochester such a memorable experience. 


\section{ABSTRACT}

The amplification of subpicosecond optical pulses using ultrashort synchronized pump pulses (synchronous amplification) is investigated in this thesis. The research presented here includes the construction and assessment, both experimental and theoretical, of a unique high power organic dye amplifier system pumped by modelocked, frequency doubled, Nd:YAG laser pulses. Advantages of this system over previous amplifier systems pumped with longer Q-switched pulses include a higher pump energy to output energy conversion efficiency and significantly reduced amplified spontaneous emission (ASE) generation. The availability of high power, wavelength tunable, subpicosecond laser pulses has opened up a number of rapidly developing research areas in time resolved Physics, Chemistry, Biology and Quantum Optics.

The design and operation of a three stage dye amplifier, which amplifies subpicosecond pulses to a peak power of 1 gigawatt, is described. Components of the system include a dye oscillator, which produces optical pulses at $605 \mathrm{~nm}$ as short as 70 femtoseconds, and two Nd:YAG laser systems, each of which is capable of preparing the ultrashort synchronized pump pulses used to drive the three stage dye amplifier. The synchronization between the pump pulse and the subpicosecond input pulse is found to be 5 ps. 
A section on dye amplifier design includes a discussion of staging, pumping geometry, spot size, dye solvent, alignment and timing. The experimental assessment of the system includes measurements of the gain, pulsewidth, ASE, amplitude fluctuations, beam quality and nonlinear index effects.

In order to optimize the synchronously pumped amplifier system, the factors that limit the gain in a single amplifier stage are investigated experimentally. The effects of saturation, and three loss mechanisms: fluorescence, molecular reorientation and ASE, are presented. Measurements of the transient gain, using a pump-probe technique, are used to investigate the loss mechanisms.

A theory that models the behavior of a dye amplifier system under ultrashort pulse excitation has been developed. Because no steady state gain assumption is included, the theory can predict the transient gain, and results are compared to experiment. The theory is also used to predict saturation behavior of the amplifier including pulse broadening. A theoretical comparison of amplifiers pumped with modelocked pump pulses and Q-switched pump pulses is also presented. 
TABLE OF CONTENTS

CURRICULUM VITAE

Page

ACKNOWLEDGEMENTS

i i

ABSTRACT

i i i

v

LIST OF TABLES

xiii

LIST OF FIGURES

xiv

CHAPTER

I .

INTRODUCTION

1

A. Intent of Thesis 1

1. Motivation 2

2. Continuum Generation 3

3. Ultrashort Pump Pulses 4

B. Literature Review 6

C. Subpicosecond Pulses 11

1. History 12

2. Frequency Domain 14

3. Detection 14

D. Amplification 15

1. Organic Dyes 15

2. Gain 16

3. Efficiency 18

4. Pump Sources 19

E. Organization of Thesis 20 
II. THE AMPLIFIER SYSTEM 23

A. Subpicosecond Amplifiers 23

1. Bell Laboratories system 25

2. California Institute of Technology System $\quad 27$

3. Other Systems 29

4. Synchronous Amplifier system 30

B. Dye Oscillator 30

1. Gain Medium and Laser Cavity 31

2. Pump Source 33

3. Frequency Doubling 35

4. Synchronous Pumping 37

5. Saturation 39

6. Saturable Absorber 40

C. YAG Amplifier System 42

1. Optically Synchronized system 43

2. Pockels Cell 45

3. Regenerative Amplifier 48

4. External Amplifier 52

5. Frequency Doubling 53

6. Temporal Jitter 58

7. RF Coupled System 61

8. Comparison of Systems 62

9. Temporal Jitter 65 
D. Dye Amplifiers

1. Parameters

1. Parameters

2. Staging

3. Saturation

4. Baseline Design

5. Dye

6. Solvent

7. Pumping Geometry and Beam Quality

8. Pump Energy

84

9. Alignment Procedure 86

10. Timing

11. Concentration

12. Spot size

13. Gains

14. Amplitude Fluctuations 96

15. Nonlinear Index Effects 97

16. Autocorrelation 99

17. Amplified Spontaneous Emission (ASE)

E. Summary

III. DYE AMPLIFIER EXPERIMENTS

A. Introduction

1. Input Parameters

2. Output Parameters 
B. Timing Curves

Page

1. Pump-Probe Experiment

113

2. Apparatus

113

3. Results

114

4. Gain Onset

116

118

C. Loss Mechanisms

119

1. Fluorescence

120

2. Molecular Reorientation

121

3. Amplified Spontaneous Emission

125

D. Transient Gain Results

1. Solvent Dependence

127

2. Spot Size Dependence

134

E. Scaling

135

F. Saturation

139

1. Comparison of Stages

140

2. Pump and Input Saturation

140

3. Apparatus

141

4. Pump Energy Results

142

5. Input Energy Results

148

G. Conclusion

152

IV. THEORY

A. Literature Review

1. Short Pulse Amplifiers

155

2. Subpicosecond Gigawatt

Amplifiers 
3. Transient Gain

B. Time Resolved Amplifier Theory

1. Initial Conditions

2. Energy Levels

3. Assumptions

4. Method of Solution

5. Cross Method

C. Calibration

1. Convergence

2. Previous Results

3. Kiton Red

D. General Amplification Curves 180

1. Input Intensity 180

2. Pump Intensity 186

E. Timing Curves 190

1. ASE Results 190

2. Comparison with Experiment 194

F. Pump Pulse Length 196

1. Simple Model 199

2. Simple Model with ASE 201

G. Pulse Shaping 201

$\begin{array}{ll}\text { H. Summary } & 207\end{array}$ 


\section{APPENDICES}

$$
\begin{aligned}
& \text { Appendix One - Electronics } \\
& \text { Appendix Two - Dye Concentration } \\
& \text { Appendix Three - Higher Lying Excited States }
\end{aligned}
$$




\section{LIST OF TABLES}

$\begin{array}{llc} & & \text { Page } \\ \text { 1-1 } & \text { Comparison of synchronous amplifier systems } & 8 \\ & \text { Comparison of gigawatt subpicosecond } & 28 \\ & \text { amplifier systems } & \\ 2-2 & \text { Pump and input parameters for the synchronous } & 95 \\ & \text { amplifier } & 108 \\ 4-3 & \text { Amplified spontaneous emission contrast ratio } & 159 \\ 4-2 & \text { Differential equations for the time resolved } & \\ & \text { theory } & 164 \\ 4-3 & \text { Parameters for kiton red } & 177 \\ 4-4 & \text { Pulse broadening due to saturation }\end{array}$


1-1 White light continuum generation 5

1-2 Synchronous amplification $\quad 17$

2-1 Optically synchronized amplifier setup 44

2-2 Regenerative amplifier pulse evolution 51

2-3 Angular dependence of frequency doubling 55

2-4 Intensity dependence of frequency doubling 57

2-5 Oscillator pulse formation 59

2-6 Comparison of synchronous amplifier systems 63

2-7 Three stage dye amplifier system 75

2-8 Comparison of pumping geometries 79

2-9 Spatial profile of the pump beam 82

2-10 Spectral characteristics of the dichroic mirror $\quad 85$

2-11 Peak gain as a function of the concentration 90

2-12 Dye amplifier relay optics 92

2-13 Experimental setup for the autocorrelation $\begin{array}{ll}\text { measurement } & 100\end{array}$

2-14 Oscillator pulse autocorrelation 103

2-15 Amplified pulse autocorrelation 105

2-16 Streak camera measurement of the amplified $\begin{array}{ll}\text { pulse and ASE } & 107\end{array}$

3-1 Experimental setup for measuring the transient gain 
3-2 Transient gain in a dye amplifier

3-3 Fluorescence dependence of the gain storage time

3-4 Polarization dependence of the gain storage time

3-5 Log of data and best fit line

3-6 Solvent dependence of the gain storage time

3-7 Pump intensity dependence of the peak gain

3-8 Comparison of gain storage time calculation and experiment

3-9 Spot size dependence of the gain storage time

3-10 Dye amplifier chain transmission

3-11 Pump intensity dependence of the first stage peak gain

3-12 Pump intensity dependence of the second stage peak gain

3-13 Pump intensity dependence of the third stage peak gain

3-14 Input intensity dependence of the first stage peak gain

3-15 Input intensity dependence of the second stage peak gain

3-16 Input intensity dependence of the third stage peak gain

4-1 Organic dye energy level diagram 
4-2 Analytical method of solution $\quad 170$

4-3 Comparison with previous theoretical results 174

4-4 Rhodamine absorption and emission spectra 175

4-5 Kiton red emission spectra 179

4-6 Input intensity dependence of the general gain curves

4-7 Input intensity dependence of the general gain curves with experimental data

4-8 Pump intensity dependence of the general gain curves

4-9 Pump intensity dependence of the general gain curves with experimental data

4-10 Theoretical calculation of the amplified pulse and ASE

4-11 Transient gain and ASE

4-12 Theory and experimental measurement of the transient gain

4-13 Small signal gain dependence on the pump pulse duration

4-14 Small signal gain dependence on the ASE

4-15 Choice of input for the pulse broadening calculation

4-16 Pulse broadening

A-1 Experimental setup of the electronics 
xvii

Page

A-2 Dye concentration dependence of the gain storage time

A-3 ASE pulse without the higher lying state absorption

A-4 ASE pulse with the higher lying state absorption 
CHAPTER I

INTRODUCTION

I.A Intent of Thesis

In this thesis, the amplification of subpicosecond laser pulses using ultrashort synchronized pump pulses will be investigated both experimentally and theoretically. Advantages of the synchronized pump pulse technique include increased efficiency and reduced amplified spontaneous emission (ASE). We have constructed two systems that produce synchronized ultrashort pump pulses. The pump pulses are used to pump a three stage organic dye amplifier system. The amplifier system is designed to preserve the subpicosecond input pulses's temporal profile while increasing the input pulse energy to the millijoule level. The design and optimization of the dye amplifier system is investigated with respect to the following objectives: 1) the system should efficiently convert pump energy to output energy, 2) it should have a good contrast ratio between the amplified pulse and the background (amplified spontaneous emission), and 3 ) it should produce good beam quality. We have investigated amplifier saturation and the loss mechanisms that limit the gain in an amplifier. In addition, a time resolved theory has been constructed which predicts the behavior of the amplifiers when they are pumped 
by ultrashort pump pulses.

Our system makes a contribution towards each of the goals listed above. of all the systems which produce gigawatt power subpicosecond pulses, ours is the only one that uses ultrashort pump pulses. As a result, we have obtained the highest efficiency and greatest contrast ratio of any gigawatt subpicosecond system. In the experimental section, we will present the first in depth study of the transient gain in a dye amplifier. In order to model the transient gain, we have also constructed the first time resolved dye amplifier theory. I will now discuss the motivation for building an amplified subpicosecond system and the implications of using ultrashort pump pulses.

\section{I.A. 1 Motivation}

New realms of investigation have been opened up by ultrashort optical pulses. Now that subpicosecond time intervals can be measured, the phenomena of interest have expanded. The motions of macroscopic objects are very slow; even objects moving at supersonic speeds move only a few angstroms in a picosecond. However, the motions of molecules and electrons can be probed. In addition, picosecond pulses offer high peak powers at small energies allowing nonlinear effects to be investigated. Because of these capabilities, ultrashort optical pulses have been used in Physics, Chemistry, Biology, Quantum Optics and 
Engineering applications.

For many applications, such as the study of nonlinear processes, high peak power pulses are required. Gigawatt pulses are needed to generate the white light continuum which extends the spectral range of subpicosecond pulses. Multiple stage dye amplifier systems have the capability of increasing the energy contained in a subpicosecond pulse above the millijoule level.

Most laser oscillators that produce subpicosecond pulses are operated close to threshold and so do not emit much energy per pulse. Techniques such as cavity dumping, which provide more energy per pulse, also broaden the output pulsewidth. The oscillator is thus required to make an ultrashort pulse of any energy and an amplifier system is used to increase the energy while preserving the temporal profile.

\section{I.A.2 Continuum Generation}

of particular interest in recent subpicosecond research is a class of nonlinear effects, including Raman and continuum generation, that require high peak powers and hence subpicosecond pulse amplifier systems. Raman generation and two photon absorption require substantially more energy than a subpicosecond pulse oscillator can deliver. Frequency doubling and white light continuum 
generation, which are useful for obtaing multiple wavelengths for a given experiment, also require an amplifier system. Of these, the white light continuum is most useful for biological and chemical applications. To generate white light, (1) a water cell is pumped by an intense subpicosecond pulse as shown in the upper portion of Figure 1-1. When the intensity is in excess of $10^{11}$ watts/centimeter ${ }^{2}\left(\mathrm{~W} / \mathrm{cm}^{2}\right)$, the spectrum of the subpicosecond pulse is broadened by four wave mixing(2) and self phase modulation (3) (Figure 1-1, lower portion). Both of these processes cease as soon as the optical driving signal is finished; hence, the new broadband pulse retains the subpicosecond pulsewidth. Thus, it is possible to obtain subpicosecond pulses throughout the visible spectrum and beyond.

\section{I.A.3 Ultrashort Pump Pulses}

The number of parameters which affect the amplification process is quite large. The most important parameter, however, is the time delay between the pump pulse and the input pulse to be amplified. Because of the use of short pump pulses, the synchronization between the pump pulse and the input pulse must be controlled to a few tens of picoseconds (ps). With the exception of a few papers that I will review shortly, the precise synchronization technique sets the present work apart from all previous work. The 


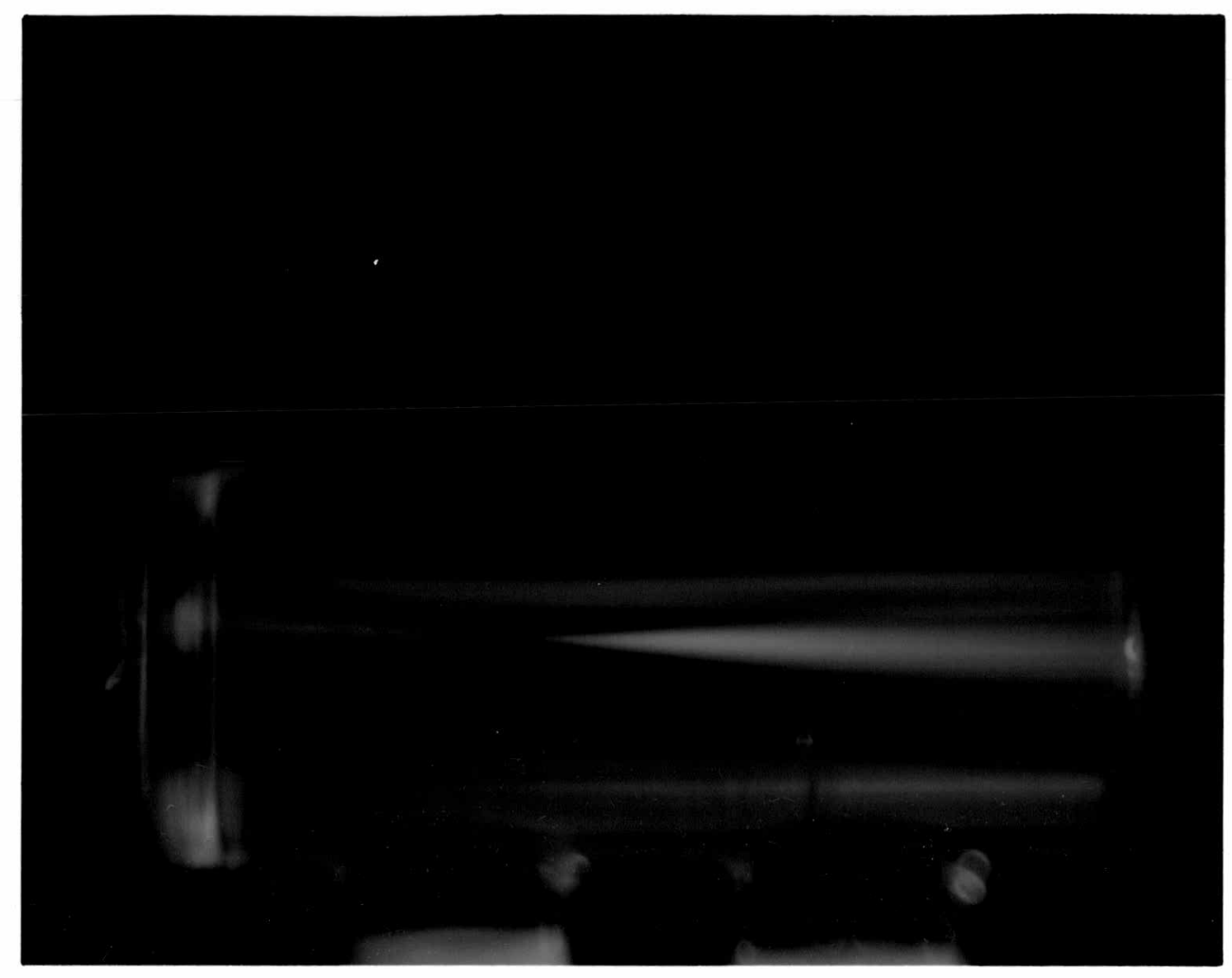

Figure 1-1 White light continuum generation

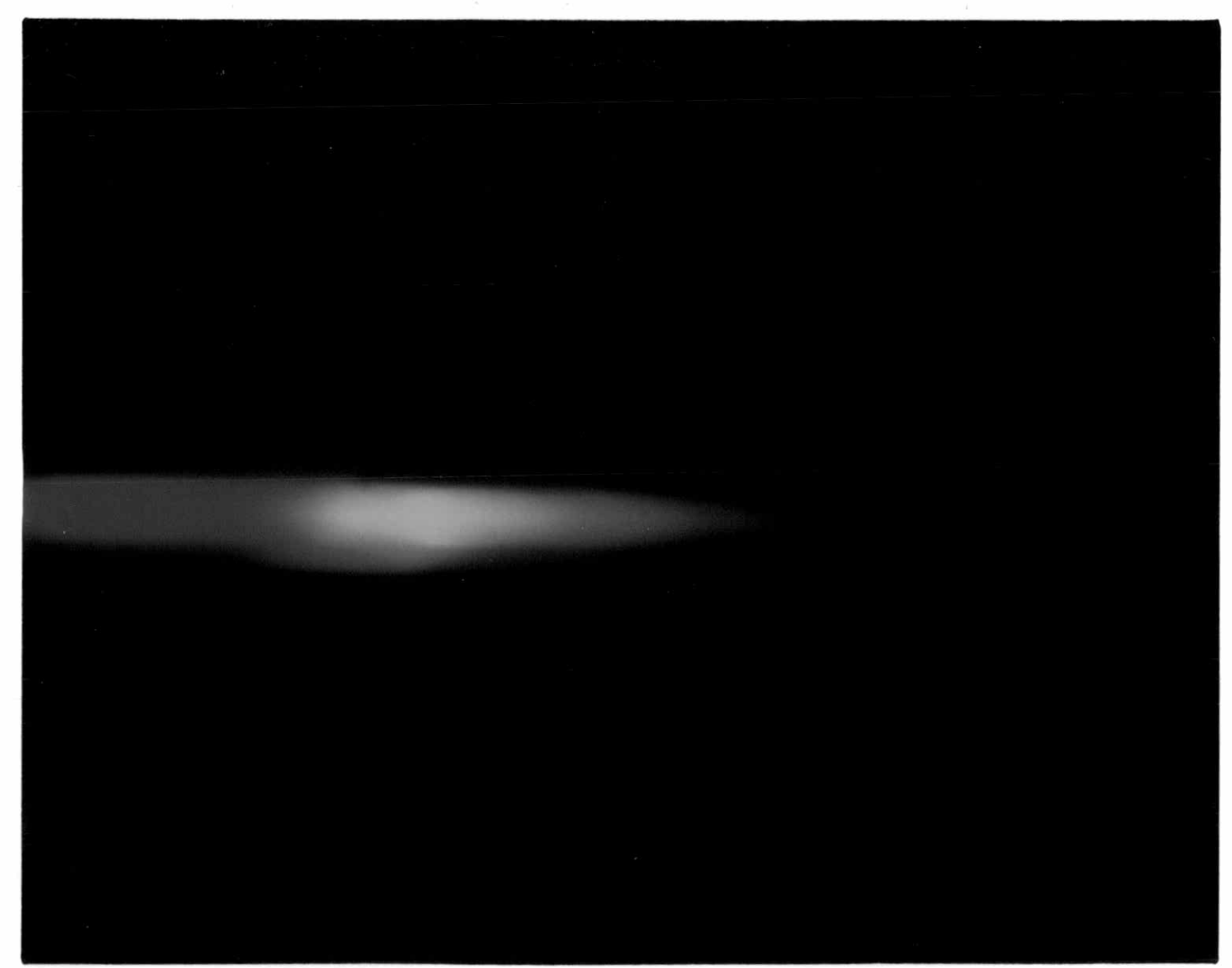


objective of precise synchronization drives the design for our system; it determines how we prepare the pump and input pulses.

Many organic dye amplifier systems have been constructed in the last two decades. At first, amplifiers were investigated in which the fluorescence time, $\boldsymbol{\tau}$, of the organic dye was much shorter than either the pump or input pulse durations. The interest then shifted to input pulses that were shorter than $\tau$, but pump pulses that were still quite long. As a result, all theoretical models could assume a steady state gain (see section IV.A). In this work we consider the case of a short input pulse and a short pump pulse. As a result, the steady state regime is never reached and the time dependence of the gain becomes the focal point of both our experimental and theoretical work.

\section{I.B Literature Review}

Flashlamps and lasers are the most common pump sources for organic dye amplifiers. Several authors (4) have used flashlamps with microsecond durations to pump dye amplifiers. Flashlamp pumped systems are convenient but due to the short gain storage time of organic dyes are very inefficient. The laser pumped dye amplifiers can be broken into two systems: nanosecond pulse pumped using a Nitrogen (5) or Q-switched laser and picosecond pulse pumped using a modelocked laser. In the present section I will 
review all of the amplifier systems which have been pumped by modelocked lasers. The amplifiers I describe below are summarized in Table $1-1$ and use picosecond optical pulses to pump organic dye amplifiers. I will refer to these systems as synchronous amplifiers. Note that of all the synchronous amplifier systems, ours has generated the highest peak power pulse. Later, in section II.A, I will review only high power dye amplifier systems, all of which amplify subpicosecond pulses using Q-switched pump lasers.

Goldberg and Moore $(6,7)$ used a frequency doubled, passively modelocked YAG oscillator with a 30 ps output pulse duration, to pump a dye oscillator, which produced 12 ps pulses. In addition, a portion of the YAG output pulse was amplified, frequency doubled, and used to pump a single dye amplifier stage. This is the first instance in the literature of a synchronous amplifier. A gain of 4 was observed with the small signal gain being 100. The amplified pulse had an energy of 60 microjoules ( $\mu J$ ) and a duration of 12 ps. The synchronism of the pump and input pulses was mentioned and the possibility of high power multiple stage systems was noted.

Huppert and Rentzepis ${ }^{(8)}$ pumped a dye cell using a frequency doubled Nd:glass system with a 6 ps output pulse duration. The active region in the dye was placed near one face of the cell which was a highly reflecting mirror. The 


\begin{tabular}{llrrrr} 
& $\begin{array}{l}\text { PUMP } \\
\text { SOURCE }\end{array}$ & $\begin{array}{c}\text { PUMP PULSE } \\
\text { DURATION }\end{array}$ & $\begin{array}{c}\text { OUTPUT PULSE } \\
\text { DURATION }\end{array}$ & $\begin{array}{r}\text { OUTPUT PULSE } \\
\text { ENERGY }\end{array}$ & $\begin{array}{r}\text { PEAK OUTPUT } \\
\text { POWER }\end{array}$ \\
\hline \hline GOLDBERG & Nd:YAG & $30 \mathrm{ps}$ & $12.0 \mathrm{ps}$ & $60.0 \mu \mathrm{J}$ & $5 \mathrm{MW}$ \\
HUPPERT & Nd:Glass & $6 \mathrm{ps}$ & $10.0 \mathrm{ps}$ & $400.0 \mu \mathrm{J}$ & $40 \mathrm{MW}$ \\
TAIRA & Nd:YAG & $18 \mathrm{ps}$ & $0.8 \mathrm{ps}$ & $1.6 \mu \mathrm{J}$ & $2 \mathrm{MW}$ \\
GOLDBERG & Nd:Glass & $5 \mathrm{ps}$ & $5.0 \mathrm{ps}$ & $1.0 \mathrm{~mJ}$ & $200 \mathrm{MW}$ \\
WOKAUN & Nd:YAG & $50 \mathrm{ps}$ & $20.0 \mathrm{ps}$ & $1.5 \mathrm{~mJ}$ & $75 \mathrm{MW}$ \\
KAFKA & Nd:YAG & $70 \mathrm{ps}$ & $200.0 \mathrm{fs}$ & $200.0 \mu \mathrm{J}$ & $1 \mathrm{GW}$
\end{tabular}

Table 1-1 Comparison of synchronous amplifier systems 
amplified spontaneous emission produced in the dye was reflected by the mirror face and traversed the active region again where it was amplified. A $500 \mu \mathrm{J}$ dye pulse of $12 \mathrm{ps}$ duration was produced with only 5 millijoule (mJ) of pump energy.

An elaborate system was developed by Taira and Yajima(9) based on a passively modelocked YAG laser. The YAG output pulse was frequency doubled and used to pump a dye oscillator which gave a 15 ps dye pulse. The dye oscillator pulse was passed through a saturable absorber and then an amplifier. The absorber had a small signal loss of 100. The dye amplifier was pumped by $18 \mathrm{ps}$ frequency doubled pulses from the same YAG laser used to pump the dye oscillator. With $3 \mathrm{~mJ}$ of pump energy a small signal gain of 100 was obtained in the amplifier. Using two absorbers and amplifiers in alternation, the dye pulsewidth was compressed down to 800 femtoseconds (fs). The mechanisms leading to compression are explained in section II.B.5. The possibility of further amplification was mentioned.

Recently Goldberg $(10)$ has used the 5 ps pump pulses produced by a frequency doubled modelocked glass laser to pump a 2 stage dye amplifier. The input pulse was a portion of the white light continuum generated by the glass laser. Gains of $10^{4}$ were achieved yielding a $1 \mathrm{~mJ}$ pulse.

During the course of the thesis work, a paper was 
published by Wokaun, Liao, Freeman and Storz(11) at Bell Laboratories. A frequency doubled, actively modelocked, Q-switched YAG laser which produced 50 ps output pulses was used to pump a dye laser oscillator and a series of 3 dye amplifiers. The oscillator produced an output pulse with an energy of $0.9 \mu \mathrm{J}$ and a duration of $20 \mathrm{ps}$. The first two amplifiers stages were pumped transversely with $3 \mathrm{~mJ}$ and $5 \mathrm{~mJ}$ of energy while the third was pumped longitudinally with $8 \mathrm{~mJ}$. The first stage had a gain of 80 while the second stage had a gain of 7. A gain of 3 is achieved in the last stage yielding a $1.5 \mathrm{~mJ}$ output pulse and a total gain of 1700 .

The authors note the following differences between their system and previous systems pumped with nanosecond optical pulses. An 88 conversion efficiency of pump to output energy is achieved with no temporal broadening of the output pulse. Conversion efficiencies up to $15 \%$ are possible but the amplified pulse is temporally broadened. The quantity of $\mathrm{ASE}$ is reduced and the amplifier stages do not need to be isolated. The timing between the pump and input pulses is critical and an experimental measurement of the transient gain curve is presented. No theory has been constructed to model the system; however, the authors attribute all of the decrease in gain to molecular reorientation (see section III.C.2). While input pulses of a much longer duration are used, the paper by wokaun is the 
only publication that is similar to the work which will be presented in this thesis.

Two trends were clear from even the earliest paper. The need for synchronization of the pump and input pulses was noted by all of the authors. In addition the trend towards higher energies was mentioned by most. Both of these concepts will be developed more fully in this thesis. I.C Subpicosecond Pulses

Subpicosecond optical pulses are attractive for the study of rapid events, such as the motions of atoms or molecules in liquids and solids. To study ultrashort events, a method for measuring time is needed. Outside of Nuclear Physics, optical pulses provide the shortest timing marks available to the scientist. Ultrashort optical timing pulses can be used directly or converted into other timing sources such as fast electrical pulses.

In the following section I will review a few highlights of the history of time measurement. Next, some of the general techniques used to characterize subpicosecond pulses are explained. We will use these techniques throughout the thesis work. 


\section{I.C.I History}

It has only been since the development of the laser that time measurements of less than a nanosecond have been possible. Historically, a number of measurement techniques have led to faster and faster measurement capabilities.

Here we will briefly review some of the milestones in time measurement, pointing out the first embodiment of techniques which are still used today.

Galileo Galilei(13) used his own heart pulse and later a pendulum to measure time. Galilei also used a jet of water flowing from a large container and then measured the weight of the water to calculate the elapsed time. Hence, the measurement of time was converted to a different quantity, in this case weight, which the experimenter could more easily determine.

To record shorter times, sound was used. In 1819 de la Tour(14) used a siren as a time standard and introduced the idea of the equivalence of a time interval and frequency.

In 1834, Wheatstone $(15,16)$ used a rotating mirror in conjunction with sparks to measure times of less than 1 microsecond $(\mu s)$. In the process, wheatstone introduced the concept of streak recording using a source of short duration.

In 1899 Abraham and Lemoine $(17,18)$ developed a new 
method called the electrical gate which used a Kerr cell and was capable of nanosecond measurement. A spark was used both to open the Kerr cell and as a source of light. The spark provided the synchronization required to avoid jitter problems.

For the next 65 years, the shortest time interval that could be measured remained about 1 nanosecond. Then came the development of the laser and finally the modelocked laser pushed time measurement into the picosecond regime. $(19,20)$ subpicosecond pulses were achieved with organic dye lasers in 1974.

The temporal measurement techniques outlined above, developed since the time of Galileo, are still in use today. Repetitive processes and the conversion of time to distance are used in the measurement of ultrashort pulses. The analysis of optical pulses in the frequency domain is important for the understanding of modelocking. Streak techniques still provide an essential mechanism for time measurement and jitter between sources remains a critical issue. Finally, it is the development of shorter timing sources that has lead the way to the study of faster phenomena. As sparks allowed the motions of speeding objects to be "frozen", so today subpicosecond optical pulses allow the observation of molecular and electronic motions. 


\section{I.C.2 Frequency Domain}

I wish to discuss a few of the topics outlined above in more detail. The frequency domain analysis is quite useful for understanding ultrashort pulses. If we let $\Delta t$ be the temporal pulse width (full width half maximum) and $\Delta \nu$ be the width of the frequency spectrum of the optical pulse, then the uncertainty principle states that the "time-bandwidth product" ( $\Delta t \Delta \boldsymbol{\nu})$ must be greater than a constant of order unity (depending on the pulse shape).(22) Thus, the bandwidth of a given laser system places a constraint on which systems are capable of producing subpicosecond pulses. For example, YAG systems have a lasing bandwidth ${ }^{(23)}$ of approximately 0.5 angstroms $(\AA)$ and can generate pulsewidths of 20 ps. (24) By comparison, organic dye lasers have lasing bandwidths on the order of $400 \AA^{(25)}$ and have generated pulses as short as $30 \mathrm{fs}$.

\section{I.C. 3 Detection}

There are no direct temporal detection means available on the subpicosecond time scale. The fastest optical streak techniques ${ }^{(27)}$ are currently limited to approximately 1 ps and, thus, indirect means must be used. Since light travels $0.3 \mathrm{~mm}$ in $1 \mathrm{ps}$, length is a convenient quantity to use. Optical path length is used to delay one pulse relative to another when performing an autocorrelation measurement (28) (see Section II.D.16). Similarly, indirect means can be 
used to investigate a material sample on the subpicosecond time scale. One pulse can be used to perturb the sample and a second pulse used to interrogate it. The polarization, attenuation or amplification of the second probe pulse yields information about the state of the sample. The time delay between the two pulses is accomplished by changing the distance that the probe pulse must travel to reach the sample. The pump-probe technique is typical of the indirect detection means used throughout this thesis.

\section{I.D Amplification}

In this section, I will review optical amplification using organic dye amplifiers. I begin by describing the organic dye gain media and the simplest model of amplification. Next, the concept of an efficient amplifier is developed and a comparison of different pump sources is presented.

\section{I.D.I Organic Dyes}

For any amplifier system, a pump source and a storage medium is required. We want to amplify subpicosecond pulses so we have chosen to use organic dyes as our storage medium which, because of their large bandwidth, have the ability to amplify the full spectrum associated with a subpicosecond pulse. In addition, organic dyes are cheap, efficient and well characterized as a gain medium. All organic dyes have 
a strong absorption band in the visible(29) due to an extended system of conjugated bonds. The absorption band is due to the transition from the ground state $s_{0}$ to the first excited state $S_{1}$. The reverse process, $S_{1}$ to $S_{0}$, is responsible for both spontaneous and stimulated emission. The emission band occurs at longer wavelengths (Stokes shifted) than the absorption band. The transition moment in organic dyes is usually quite large.

An intense light source, such as a flashlamp or laser, is used to pump the dye. The organic dye should have an absorption band that closely matches the pump source. We have chosen to use as our pump source a frequency doubled Nd:YAG laser. The active medium is drawn from the most often used class of dyes, the Xanthenes. (30) The Xanthene dyes emit in the range from $500 \mathrm{~nm}$ to $700 \mathrm{~nm}$ and include the dyes rhodamine $6 \mathrm{G}$ and kiton red.

\section{I.D. 2 Gain}

The most important task of an amplifier is to provide gain for the input pulse. Figure 1-2 shows a dye cell with both a pump pulse and an input pulse incident. Some of the energy from the pump pulse pumps the ground state, $\mathrm{s}_{0}$ ' molecules into the first excited state, $S_{1} \cdot$ The input pulse then comes through the amplifier and stimulates emission from these excited molecules. In this manner, the input pulse is amplified. The simplest rate equation model of the 


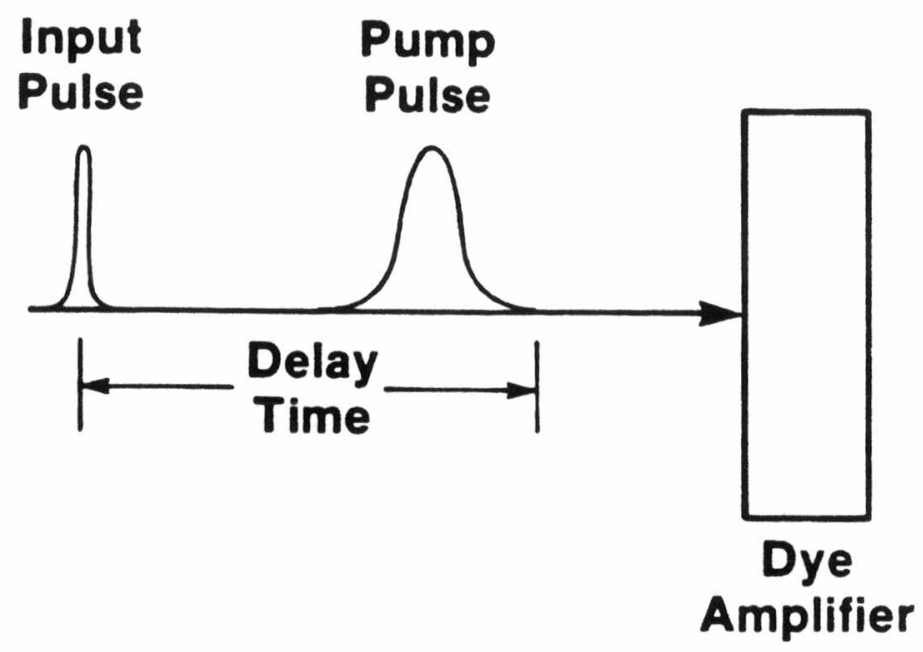

Figure 1-2 Synchronous amplification 
process gives the following formula for the amplification of the input pulse:

$$
\mathbf{G}=\mathbf{e}^{\boldsymbol{\sigma N} \ell} \text {, }
$$

where $G$ is the gain, $\sigma$ is the emission cross section of the dye, $\mathbf{N}$ is the number of excited molecules per unit volume and $l$ is the length of the amplifier. The gain, $G$, of an amplifier is defined as the ratio of the output pulse energy to the input pulse energy. In the simplest case, $\mathrm{N}$ is directly related to the pump energy where each pump photon is responsible for producing one excited dye molecule. Realistic modifications to the simple rate equation model will be made throughout the thesis; however, it is often useful to refer to the above result to develop an intuitive feeling of the situation.

\section{I.D.3 Efficiency}

The efficiency of amplification depends on the dye quantum efficiency, the gain storage time of the dye and the synchronization between the pump and input pulses. Dyes have inherent losses in converting pump energy to output energy. The quantum efficiency is a measure of this conversion and for organic dyes typically has a value of $908 ;(31)$ much higher than most amplifier media. Unfortunately, dye amplifiers maintain their gain for extremely short times after being pumped due to rapid 
losses. The gain storage time is defined as the time for the gain to decrease to $1 / \mathrm{e}$ of its initial value. The mechanics of the rapid losses that deplete the stored energy will be discussed in detail in Chapter III. The major result is that most of the energy in a high gain dye amplifier is lost within a nanosecond after it is deposited. For the entire pump energy to be stored by the dye, the energy must be deposited in less than a nanosecond, that is, before the losses remove it. The input pulse must then be immediately incident on the amplifier. The pump and input pulses must be synchronized to better than one nanosecond in order to achieve a stable, high power output pulse.

\section{I.D. 4 Pump Sources}

Many different pump sources have been used to excite dye amplifiers. Flashlamps deposit their energy on the millisecond to microsecond time scale. Nitrogen and Q-switched YAG lasers generate pump pulse widths on the order of $10 \mathrm{~ns}$. It is the frequency doubled, Q-switched YAG which has found the greatest use as a pump source for high gain dye amplifiers to date. Q-switched YAG systems feature high powers, high efficiencies and good availability. While not all of the energy in the 10 ns pump pulse can be deposited simultaneously, the synchronization between the pump and input pulse need be only as good as 1 ns. Fortunately 1 ns accuracy can be achieved with electronic 
synchronization between the lasers that generate the pump and input pulses. The Q-switched laser does not produce a transform limited pulse. As a result, the pump pulse may not be gaussian in time and can exhibit a modulated temporal profile. (32) The gain of the amplifier will follow these fluctuations (33) in the pump pulse and give rise to large fluctuations in the amplitude of the amplified pulse.

In our synchronous amplifier system we use nearly transform limited 70 ps pump pulses to excite the dye amplifiers; hence, synchronization of better than $70 \mathrm{ps}$ is required. Two systems that provide the required synchronization have been constructed. (34) One is called the RF synchronized system and is accurate down to 40 ps jitter. The other is the optically coupled system which has a jitter between the pump and input pulse of less than a few picoseconds. The combination of short, synchronized pump pulses yields a more efficient system than those employing $10 \mathrm{~ns}$ pump pulses. Using $70 \mathrm{ps}$ pump pulses, almost all the pump energy is stored in the dye at one time. Using synchronized input pulses, the stored energy can be accessed at its peak before significant losses have occured.

\section{I.E Organization of Thesis}

Following the Introduction, Chapter II deals with the construction of the high power, subpicosecond pulse amplifier system. First the state of the art of such 
systems is reviewed. Next the dye oscillator system for generating the subpicosecond pulse is described. The following section contains a description of the systems that have been constructed for preparing a synchronized pump pulse. The philosophy behind the dye amplifier system is presented as well as a detailed description of the three stage amplifier system. The alignment proceedure is described followed by prescriptions for optimizing the important parameters. Finally, the output characteristics of the amplifier system are discussed.

Chapter III contains the results of experiments aimed at characterizing the dye amplifier system. After an introduction, the measurement of the transient gain is described. Three major loss mechanisms are identified and their effects predicted. The first section concludes with a series of transient gain measurements which verify these predictions. In the second portion of the chapter, the difference between each of the stages is emphasized. The effects of gain saturation are explained and then illustrated experimentally.

The theory is contained in Chapter IV. First a survey of the literature is given and the need for a new time resolved theory is identified. The theory, including assumptions and method of solution, is then detailed. Next the results are compared to the experimental results given 
in Chapter III. Finally the theory is used to predict the efficiency of a dye amplifier system as a function of pump pulse duration. In addition, the effects of gain saturation on input pulse width are modeled.

Throughout this thesis we have given sufficient detail so that the amplifier system can be duplicated. Moreover, it is hoped that the physics behind the design is made sufficiently clear so that the reader can design a system given different constraints. 


\section{II.A Subpicosecond Amplifiers}

For many of the applications mentioned in Chapter I, a subpicosecond dye amplifier system should have the following features. The amplified pulsewidth should be ultrashort, approximately $100 \mathrm{fs}$. The output energy should approach 1 mJ yielding a peak power of over 1 gigawatt. A low noise system, one with a large contrast between the output pulse and the amplified spontaneous emission (ASE) background, is required. Also required is good output beam quality so that the amplified pulse can be focussed to a small spot. The efficiency of conversion from the pump energy to output energy should be a few per cent or more.

For the amplifier system to be useful for a wide variety of experiments, the output pulse should also be tunable over a broad band of wavelengths. In addition, a pulse repetition rate of at least a few hertz is needed for experiments that require signal averaging. The output parameters of the amplifier should be stable and the performance reliable. Finally, the subpicosecond amplifier should be as inexpensive as possible, given the above constraints. 
The design decisions detailed in this chapter are aimed at achieving the goals listed above. I will describe the system we have constructed for producing amplified subpicosecond pulses. To begin with, I will review the other systems that have been constructed to produce high power, ultrashort pulses. Our system differs fundamentally by using short synchronized pump pulses. The main consequences of using 70 ps synchronized pump pulses is the increased efficiency of the amplifier system and the decrease in the amount of background ASE. One major restriction is that the pump and input pulses must now be well synchronized. Using short, synchronized pump pulses will change many of the system parameters. Descriptions are given of the systems that prepare the pump pulses and the oscillator which prepares the subpicosecond input pulses. The design and operation of the dye amplifiers is then discussed fully.

In Chapter I, I reviewed all of the synchronous amplifier systems which used short synchronized pump pulses to excite the amplifiers. None of these systems, except for ours, had reached the gigawatt level, and only one other used subpicosecond pulses. In the following literature review section, I will examine those systems which have amplified subpicosecond pulses to the gigawatt level. All of these systems use nanosecond pump pulses, generated by a Q-switched YAG laser, to excite the amplifiers. 
The number of gigawatt subpicosecond laser systems is approaching ten in number at this writing. Understandably, there are few complete descriptions of how to build such a system in the literature. In addition, since each laboratory has chosen a different set of constraints to work within, the actual designs are varied and seem almost contradictory at times. The constraint which most separates the present work from all previous designs is the use of modelocked pulses to pump the amplifier, not Q-switched pulses. It is none the less important to understand how other workers have approached the construction of a system that meets the requirements listed above.

\section{II.A.I Bell Laboratories System}

The first amplified subpicosecond pulse system was built at Bell Laboratories by Charles Shank and Erich Ippen. The most complete description of the amplifiers for the system is given in a paper by Migus, Shank, Ippen and Fork. (35) It is from this paper that I will review their design.

Their oscillator is a passively modelocked dye laser pumped by a CW Argon laser. The oscillator generates an input pulse which has an energy of $2 \mathrm{~nJ}$ with a $500 \mathrm{fs}$ temporal width at a wavelength of $615 \mathrm{~nm}$. A frequency doubled, Q-switched YAG is used to provide the pump pulse which has a duration of $8 \mathrm{~ns}$ and an energy of $120 \mathrm{~mJ}$. As a 
result of the relatively long 8 ns pump pulse, a jitter between the oscillator pulse and the pump pulse of approximately $1 \mathrm{~ns}$ is allowed and the synchronization is accomplished electrically.

In the paper, Migus develops a theory for an amplified pulse which is short compared to the pump pulse duration. He states that the amplification is dependent on the steady state excited population in the dye. The population is dependent on the rate of generation of excited molecules and the depletion rate due to amplified spontaneous emission (ASE). The pump pulse must be much longer than the response time of the dye in order that a steady state situation be reached. The steady state assumption is true for 8 ns pump but not for 70 ps pump pulses which is an important difference between our system and the Bell Labs system.

Migus calculates the stored energy in the amplifier and the saturated gain of the amplifier for a series of input pulses. From the calculations he concludes that because of saturation and ASE, a single stage system will not provide the required gain and so a three stage system is used. The first two stages are pumped transversely and the third longitudinally. The choice of dyes are $5 \times 10^{-4}$ molar (M) kiton red for the first stage and $1 \times 10^{-4} \mathrm{M}$ and $1 \times 10^{-5} \mathrm{M}$ rhodamine 640 for the last two. The choice of dye is dependent on both the wavelength and the pumping geometry. 
The pump energy for each stage and the spot size, which is the size of the active pumped region in the amplifiers, are shown in Table 2-1 along with the output energies.

Several means were used to isolate the stages from each other in order to prevent ASE from propagating down the amplifier chain. A spatial filter and a spectral filter separate the first two stages while a flowing jet of the saturable absorber malachite green provides isolation between the last two stages. Without isolation between the stages, the ASE would completely swamp the input pulse. The requirement for isolation is also an important difference between their system and the one we have built.

Since the publication of the paper by Migus et al., many upgrades have been made ${ }^{(36)}$ to their system. The oscillator has been changed to a colliding pulse modelocked (CPM) system yielding 70 fs input pulses. The amplifier system has now been increased to four stages. The first three stages are transversely pumped while the last is longitudinally pumped. Compression of the amplified pulse in an optical fiber and grating pair has produced a record 30 fs output pulse. (26)

II.A.2 California Institute of Technology System

Only one other system has been described in detail. The system was built by Koch, Chiu and Yariv at the 


\begin{tabular}{lrrr} 
& $\begin{array}{c}\text { SPOT } \\
\text { SIZE }\end{array}$ & $\begin{array}{r}\text { PUMP PULSE } \\
\text { ENERGY }\end{array}$ & OUTPUT \\
\hline \hline MIGUS & & & \\
1st Stage & $250.0 \mu \mathrm{m}$ & $6 \mathrm{~mJ}$ & $1.0 \mu \mathrm{J}$ \\
2nd Stage & $2.5 \mathrm{~mm}$ & $18 \mathrm{~mJ}$ & $100.0 \mu \mathrm{J}$ \\
3rd Stage & $7.0 \mathrm{~mm}$ & $96 \mathrm{~mJ}$ & $1.5 \mathrm{~mJ}$ \\
& & & \\
KOCH & & & \\
1st Stage & $2.5 \mathrm{~mm}$ & $8 \mathrm{~mJ}$ & $10.0 \mu \mathrm{J}$ \\
2nd Stage & $2.5 \mathrm{~mm}$ & $8 \mathrm{~mJ}$ & $42.0 \mu \mathrm{J}$ \\
3rd Stage & $5.5 \mathrm{~mm}$ & $60 \mathrm{~mJ}$ & $700.0 \mu \mathrm{J}$
\end{tabular}

Table 2-1 Comparison of gigawatt subpicosecond 
California Institute of Technology. (33) Their oscillator is a synchronously pumped dye laser using a modelocked argon laser as the pump. The dye amplifier consists of three longitudinally pumped stages. A Q-switched YAG is used to pump the amplifiers with a 15 ns duration pulse. The dye rhodamine 640 is used in all stages and always at the concentration of $10^{-5} \mathrm{M}$. The spot size, pump energy and output for each stage are shown in Table 2-1. Saturable absorbers but no spatial filters are used to isolate the dye amplifier stages from each other. The system was designed with simplicity in mind and the compromises made account for a small decrease in the system efficiency. For instance, note the large spot size used in the first stage in order to make the first two stages identical. The increased spot size creates a lower input intensity than optimal in the first stage, which reduces the efficiency.

II.A.3 Other systems

Other gigawatt subpicosecond systems have been constructed by Ippen, Kenney-Wallace, and Wilson. (37) Although none have been published, these gigawatt subpicosecond systems are either copies of our system or the system at Bell Labs. For the systems in the latter catagory, the following generalizations can be made. A CW or a modelocked Argon laser is used to pump a dye laser oscillator. All of these systems amplify a subpicosecond 
pulse by a factor of at least $10^{6}$. Most use three or four stages pumped by a frequency doubled Q-switched YAG laser with approximately a $10 \mathrm{~ns}$ pulse duration. All systems require isolation between the stages to prevent ASE from propagating down the amplifier chain. Finally, the efficiency of conversion from pump energy to output energy is approximately 18 or less (see Table 2-1).

II.A.4 Synchronous Amplifier System

The system I will describe changes many of the parameters described above. A CW modelocked YAG is used to pump the dye oscillator, and a frequency doubled modelocked YAG with a pulse duration of $70 \mathrm{ps}$ is used to pump the amplifiers. The synchronization between the oscillator and amplifiers is a major issue. Our system requires no isolation between stages to suppress ASE and has an efficiency as high as $10 \%$.

II.B Dye Oscillator

All systems which have been built to produce high power picosecond pulses consist of two major subsystems: the oscillator and the amplifier. The purpose of the oscillator is to produce a short pulse which is repeatable in pulsewidth and amplitude. We have constructed a dye oscillator which, under optimum conditions, produces pulses of $70 \mathrm{fs}$. (38) Typical values for the oscillator pulse width 
are $100 \mathrm{fs}$ to $200 \mathrm{fs.} \mathrm{Parameters} \mathrm{such} \mathrm{as} \mathrm{wavelength,}$ polarization and output energy are chosen in order to obtain maximum stability and minimum pulse width. The purpose of the amplifier is to increase the energy contained in the oscillator pulse with the restriction that the pulse width must not increase. However, the oscillator and the amplifier are not independent; it is the temporal synchronization of the two which is of critical importance. In the remainder of this chapter, I shall describe in detail the high power subpicosecond pulse laser system that has been constructed. The design of our system differs substantially from previous systems. The pump pulse is shorter, the accuracy of synchronization between the oscillator and amplifier is greater, the efficiency is higher and the quantity of ASE generated is less.

II.B.I Gain Medium and Laser Cavity

Most lasers consist of three parts: a pump source, a gain medium and a cavity to provide optical feedback. Recall from Chapter I that in order to produce ultrashort pulses, a medium with a large gain bandwidth is required. Lasers using organic dyes, F-centers, Alexandrite, Excimers and other broad bandwidth gain media are all capable of producing picosecond or shorter pulse widths. We have chosen to use organic dyes since they are presently the simplest and best understood of these systems. 
The most common choice of dye, and one of the most efficient, is the dye rhodamine 6G. The rhodamine $6 \mathrm{G}$ is mixed with the saturable absorber dye DQOCI. Ethylene glycol is used as the solvent and a flowing dye jet (Coherent) is used to obtain a $200 \mu \mathrm{m}$ thick free flowing stream of dye. The flow system consists of a Micropump pump with an Acroflow $0.8 \mu \mathrm{m}$ filter to remove air bubbles from the system. Both fine and coarse controls are provided to adjust the pressure of the dye and thus the thickness of the stream. The cavity configuration used is the standard astigmatically compensated cavity of Kogelnik et al.(39) Two focussing mirrors are used to obtain a small active region in the dye jet. A $2.5 \mathrm{~cm}$ radius mirror is used as one end mirror while a $5 \mathrm{~cm}$ radius mirror collimates the laser beam and sends it to a flat output mirror. The curved mirrors have broadband high reflectivity dielectric coatings while the output mirror has a 958 reflective coating.

Provision is made to vary the total cavity length with an accuracy of less than 1 micron. The reasons for such accuracy will be explained below. An Aerotech mount is used for the coarse adjustment and a Burleigh piezoelectric transducer is used for the fine adjustment. The entire cavity is mounted on a slab of super-invar so that the cavity length will be stable even with typical temperature variations $(\approx 5 \stackrel{\circ}{\circ})$. 
Finally, an $8 \mu \mathrm{m}$ thick pellicle, which serves as a Fabry-Perot etalon, is used as a tuning element. The very thin etalon is needed to provide a large free spectral range and, hence, avoid any limit to the lasing bandwidth by the tunning element. The lasing bandwidth must be maximized in order to obtain ultrashort pulses. In addition, the dispersion of the cavity must be kept to a minimum and, therefore, all glass has been eliminated from the cavity.

\section{II.B.2 Pump Source}

Since we have already chosen the gain medium, we must now choose a pumping source which will couple energy into the medium efficiently. The most efficient source is a laser with its emission line in the green where rhodamine 6G absorbs. The most common choice is an Argon laser; however, a frequency doubled Nd:YAG system offers many advantages. (34) A YAG based system offers the ability to couple the oscillator and amplifier systems optically and temporally because it has sufficient energy to pump both. In addition, the YAG system produces shorter pump pulses for the dye oscillator. On a practical level the YAG system is more rugged and cheaper to maintain than the Argon system. The YAG system used must produce modelocked pulses so that the frequency doubling process will be efficient and so that the dye oscillator can be synchronously pumped. In addition, the frequency doubled YAG pulses should be 
continuous (CW) in order to obtain the best synchronous pumping as will be described below.

The YAG source we use is a Quantronix Model $114 \mathrm{CW}$ modelocked Nd:YAG laser. The cavity consists of a high reflectivity $100 \mathrm{~cm}$ radius and an $88 \%$ reflective flat output coupler. The YAG rod is $3 \mathrm{~mm}$ wide by $65 \mathrm{~mm}$ long and is pumped by a single krypton arc lamp. The power supply has been modified in order to obtain a more stable current to the lamp. The cavity contains a Brewster plate to obtain a polarized beam and a shutter. Modelocking is accomplished with a water cooled acousto-optic transducer. The transducer is driven by a Rockland Model 5600 frequency synthesizer and a ENI model 320L amplifier. The synthesizer and amplifier provide a very stable 10 watt radio frequency signal at $50 \mathrm{MHz}$ to the modelocker. The modelocker induces a variable loss in the cavity with a minimum loss occuring once every $10 \mathrm{~ns}$. The cavity round trip time is adjusted with an Aerotech adjustable positioner on one end mirror. The round trip time is set precisely equal to the period of the $50 \mathrm{MHz}$ driving signal.

When the laser is turned on, optical noise bursts are produced. Some of the noise bursts happen to pass through the modelocker during its time of minimun loss and, hence, see a smaller loss. All noise bursts arriving at the modelocker at other times are attenuated to some greater 
degree. After multiple passes through both the gain medium and the loss of the modelocker, a single pulse evolves. In our system the duration of the infrared pulse is approximately 100 ps. A minimum for the pulse width is obtained when the cavity length has been optimized. An individual $1064 \mathrm{~nm}$ pulse has an energy of 80 nanojoules $(n j)$. The repetition rate of the laser is $100 \mathrm{MHz}$ or one pulse every $10 \mathrm{~ns}$, giving an average power of 8 watts (W).

\section{II.B. 3 Frequency Doubling}

Two important criteria in choosing a frequency doubling crystal are the conversion efficiency and the damage threshold. The conversion should be as efficient as possible; however, it is the damage due to infrared absorption that severly limits the selection of crystals. In many cases the peak power causes the damage. In our case it is the average power of $8 \mathrm{~W}$ that damages the crystal. Only two crystals exist with low enough absorption at 1064 nm so that they are able to withstand the incident power. Unfortunately, both are difficult to obtain commercially.

The first crystal is $\mathrm{Ba}_{2} \mathrm{NaNb}_{5} \mathrm{O}_{15}$ termed "banana". It is a temperature tuned crystal and was used for the experiments described in section III.A through III.D. The input beam is focussed to a $150 \mu \mathrm{m}$ spot in the $5 \mathrm{~mm}$ long crystal in order to get a conversion efficiency of approximately 58. "Banana" has an absorption (40) of 0.28 at 
$1064 \mathrm{~nm}$ but has an absorption of 48 at $532 \mathrm{~nm}$, the second harmonic wavelength. The low absorption at $1064 \mathrm{~nm}$ is needed to avoid damage; however, the absorption at $532 \mathrm{~nm}$ causes temperature detuning of the crystal. In the region occupied by the optical beam, the crystal is heated by the absorption of the green light. The temperature change then causes a change in the conversion efficiency of the temperature tuned crystal. It is this feedback that causes the temperature versus doubling efficiency curve to exhibit hysteresis. In practice, the optimum operating temperature for the crystal has to be approached from the high side in order to avoid hopping past it. In addition, any fluctuation in the crystal temperature or incident optical power causes the crystal to "turn off."

With care, the "banana" crystal works reliably and produces $500 \mathrm{~mW}$ of energy at $532 \mathrm{~nm}$ contained in a continuous train of $70 \mathrm{ps}$ pulses at $100 \mathrm{MHz}$. This is the pump source for the oscillator.

Because of the stability problems with a temperature tuned doubling crystal, an angle tuned crystal was sought with the ability to withstand the $8 \mathrm{~W}$ of average power at $1064 \mathrm{~nm}$. For the experiments described in section III.F, the doubling crystal $\mathrm{KTiOPO}_{4}$ (KTP) was used. The KTP crystal has a higher damage threshold than "banana" so the beam size in the crystal can be reduced yielding a higher 
intensity per pulse and correspondingly a higher conversion efficiency. One watt of $532 \mathrm{nn}$ light is produced for a $7 \mathrm{~W}$ incident $1064 \mathrm{~nm}$ beam. In addition, the system is more stable since the temperature sensitivity of the crystal has been greatly reduced.

\section{II.B.4 Synchronous Pumping}

So far, I have discussed the choice of gain medium, cavity and pumping source for the dye oscillator. The method employed to generate the ultrashort dye pulses in the dye oscillator is called synchronous pumping. (41) Unlike the YAG pump laser, where the modelocking of the $1064 \mathrm{~nm}$ pulses is accomplished by periodically varying the cavity loss, synchronous pumping achieves modelocking of the dye laser by periodically varying the gain. The $532 \mathrm{~nm}$ pump energy arrives at the organic dye gain medium in 70 ps bursts once every $10 \mathrm{~ns}$. The exact repetition rate is determined by the modelocking frequency and the cavity length of the YAG pump laser. Once the pump pulse arrives at the dye jet, the gain is established and spontaneous emission begins. The round trip time of the dye oscillator cavity is set to be exactly equal to that of the YAG pump laser. When the spontaneous emission returns to the dye jet, it finds that the next pump pulse has just arrived and, hence, there is gain present. The spontaneous emission is then amplified until a steady state condition is reached. 
The steady state condition is achieved when the amplified spontaneous emission forms a pulse which is energetic enough to saturate the gain and reduce the gain to a level equal to the cavity losses. The dye pulse propagates around the cavity being amplified in the gain medium and then being partially coupled out of the cavity at the output mirror.

The steady state modelocked condition is stable as long as the YAG pump laser and dye oscillator cavities are equal in length. (42) If the dye cavity is too short, the dye pulse returns to the dye jet before the gain is established and the pulse dies out. If the dye cavity is too long, the dye pulse arrives at the jet after the pump pulse. This situation is not as severe since some gain still remains even after the pump pulse is over. If the cavity is only slightly too long, the dye pulse will be reshaped by the gain and the situation will reach a steady state. However, if the dye cavity is much too long, the dye pulse becomes progressively later and later with respect to the pump pulse and the laser will turn off. The cavity mismatch tolerance is therefore asymmetric ${ }^{(42)}$ and depends on the cavity lifetime as well as the dye pulse width. Within the acceptable range of cavity lengths, the oscillator will continue to lase; however, the pulsewidth will vary greatly. Typical values for the cavity mismatch tolerance are from $1 \mu \mathrm{m}$ to $5 \mu \mathrm{m} .(43)$ 


\section{II.B.5 Saturation}

By employing synchronous pumping, a significant reduction in the dye oscillator pulse width is obtained compared to the pump pulse width. The pulse shortening is due to the balance between two processes: saturable absorption and saturable gain. (44) The gain of the dye jet is not a fixed number but depends on the incident energy of the pulse to be amplified. The gain remains constant for small values of input energy but is reduced when the input dye pulse is large enough to saturate the gain medium. Saturation occurs because there is a finite amount of stored energy in the jet and when a significant portion is removed by amplifying the pulse, the gain is reduced. Thus the gain decreases slightly while the dye pulse is passing through the dye jet. Since the gain is greater for the peak than it is for a point half way down the trailing edge, saturable gain causes the trailing edge of the input pulse to be shortened. (45)

Saturable absorption operates in an analogous manner on the leading edge of the pulse. Part of the absorption band of the gain medium overlaps the emission band causing the dye pulse to see some absorption in the jet. There are, however, only a finite number of absorbing molecules in the jet and so the leading edge of the dye pulse is absorbed more strongly than the trailing edge of the pulse. In this 
manner the front edge of the dye pulse becomes shortened.(45) The combination of saturable gain and saturable absorption can cause a very significant overall shortening of the dye pulse if the number of trips through the dye jet is large. We take maximum advantage of these effects by continuous pumping of the oscillator which allows the dye pulse to reach a steady state. Systems which employ a pump that is not $C W$ generally produce a pulse shortening factor of less than 10. (11) For CW pumped systems the pulse shortening factor is on the order of 100.

\section{II.B. 6 Saturable Absorber}

The saturable absorption can be enhanced leading to further pulse shortening. By adding a saturable absorber dye to the rhodamine $6 G$ gain dye, the magnitude of the effect can be varied. The dyes cresyl violet, DODCI, and DQOCI were all investigated with DQOCI giving the best results. As the concentration of the absorber is increased, the output pulse shortens dramatically; however, the output power also decreases. The decrease in power is due to the absorber dye which adds a linear loss as well as a saturable loss. When the concentration of the absorber dye becomes too high, the laser drops below threshold. Experimentally, the optimal concentrations were determined to be $5 \times 10^{-3} \mathrm{M}$ rhodamine $6 \mathrm{G}$ and $5 \times 10^{-4} \mathrm{M}$ DQOCI. With the combination of active modelocking (synchronous pumping) and passive 
modelocking (saturable absorbers) output pulses as short as 70 fs have been obtained.

The effect of the saturable absorbing dye can also be viewed in the frequency domain. The shortest pulses are obtained when the laser is tuned to $605 \mathrm{~nm}$, close to the absorption peak of the absorber. Although the cavity has all the glass elements removed, there are still some dispersive elements: in particular, the dielectric coatings on the mirrors and the solvent for the dye jet. The dispersion ultimately limits the pulse width since it causes the red portion of the broadband (100 ̊) subpicosecond pulse to lead the blue portion, broadening the pulse.

The absorber, however, is operating near its absorption maximum and therefore exhibits anomalous dispersion. The anomalous dispersion causes the blue portion of the broadband dye pulse to catch up and partially cancels the dispersion of the cavity. In the frequency domain the absorber can thus be seen as shortening the dye pulse by reducing the overall dispersion in the cavity. It should be possible by a judicious choice of several dyes to make a dispersionless cavity over a large bandwidth and thus reduce the pulsewidth further. If the dispersion is compensated, the dye pulsewidth will only be limited by the available bandwidth of the dye $(\approx 400 \AA)$. 


\section{II.C Yag Amplifier system}

The function of the dye amplifier system is to increase the energy contained in the subpicosecond dye oscillator pulse without distorting the temporal pulse shape or the spatial profile. The function of the YAG amplfier system is to provide a synchronized pump pulse for the dye amplifiers. As explained in Chapter I, dye amplifiers are not able to store energy over long periods of time. Thus, for a system which efficiently converts the pump energy to output energy, we require as short a pump pulse as possible. The requirement necessitates accurate temporal synchronization between the pump pulse and the pulse to be amplified. We have constructed two YAG amplifier systems which exhibit the required synchronization and a detailed description of these systems follows.

The dye amplifier system, like the oscillator requires a gain medium and a pump source. The exact choice of the gain medium will be discussed in detail in section II.D.5; however, it is important to note that the gain medium for the amplifier should be similar to the gain medium for the oscillator. The similarity is necessary because we require a peak gain at the same wavelength as the oscillator and require a broadband amplifier in order to preserve the temporal pulse shape. Because we have chosen to use an organic dye that emits in the red as the gain medium, we 
require a pump pulse with a wavelength of approximately $550 \mathrm{~nm}$ as described in section I.D.I.

II.C.I Optically Synchronized system

The first amplifier pumping system employs optical synchronization between the YAG pump pulse and the dye oscillator input pulse. In the optically synchronized system both the pump and input pulses are derived optically from the same source. The source, which functions as the clock for the entire system, is the $\mathrm{CW}$ modelocked Nd:YAG laser described in section II.B.2, which pumps the oscillator. A portion of the YAG laser output beam is split off and amplified. The amplified $1064 \mathrm{~nm}$ pulses are frequency doubled to produce the required green pump pulses. since the pump pulses are derived from the same $C W$ modelocked laser that drives the oscillator, they are well synchronized to the input pulses generated by the dye oscillator. The temporal jitter between the pump and input pulses will be calculated in Section II.C.6. An upper limit for the jitter is established experimentally in section III.D. 2 .

The layout of the pump pulse preparation system is shown in Figure 2-1. The $532 \mathrm{~nm}$ pulses are separated from the $1064 \mathrm{~nm}$ pulse after the first doubling crystal with a dichroic mirror. The $1064 \mathrm{~nm}$ pulses then pass through a double pockels cell switchout. (46) The pockels cell 


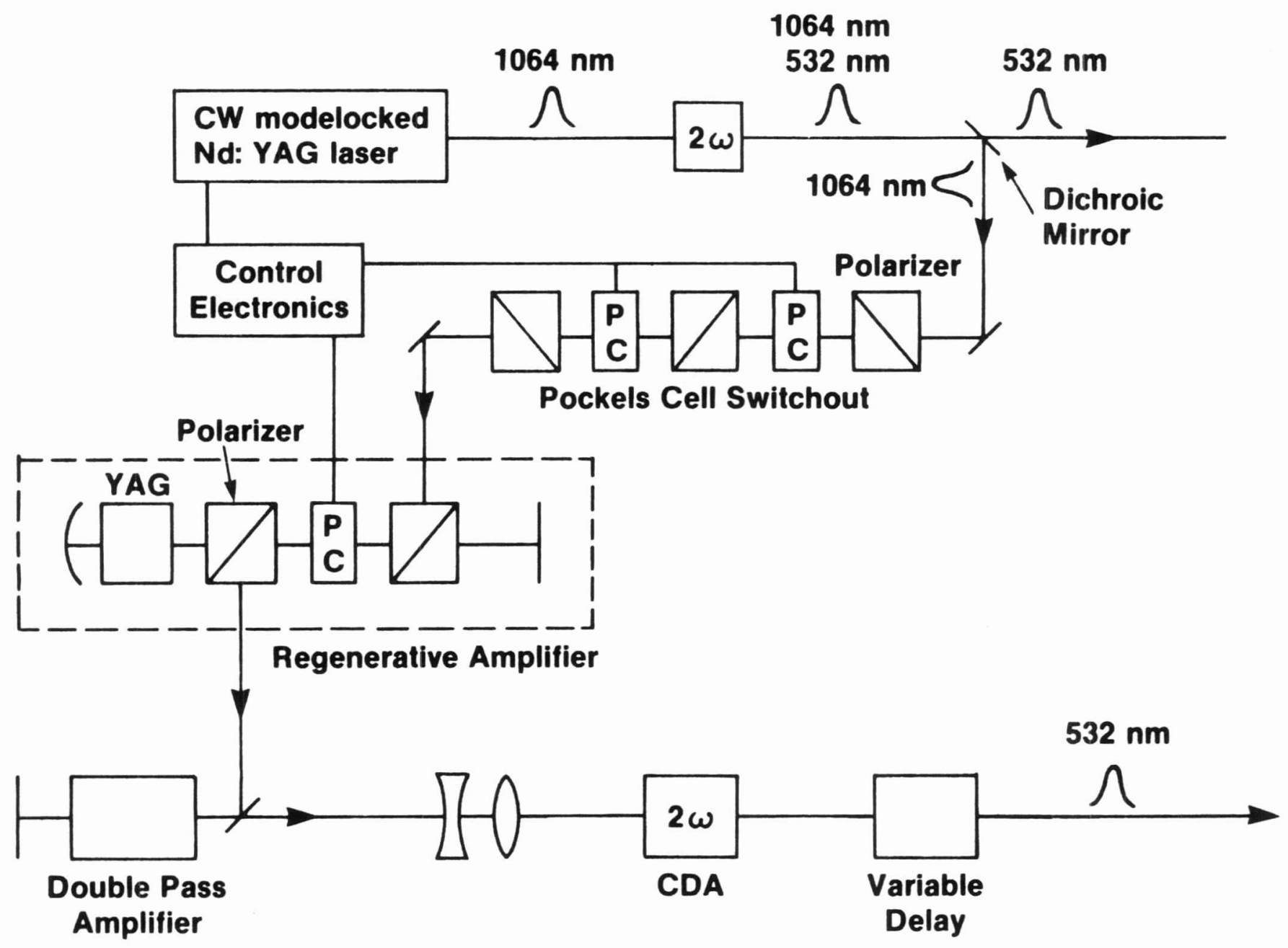

Figure 2-1 Optically synchronized amplifier setup 
performs two functions. The first function is to switchout a single pulse to be amplified from the $100 \mathrm{MHz}$ stream of pulses coming from the YAG oscillator. The remaining portion of the pump pulse preparation system is restricted to a repetition rate of $10 \mathrm{~Hz}$ by the cooling requirements of the YAG amplifier heads. The second function of the Pockels cell is isolation. The transmission window created by the Pockels cell is only $10 \mathrm{~ns}$ wide. Any reflection from the latter portion of the amplifier system is rejected by the Pockels cell and prevented from passing backwards to the oscillator. Isolation is important since a focussing lens is used before and after the oscillator doubling crystal ("banana"); hence, any returning beam would be focussed into the crystal and could easily exceed the damage threshold. In addition, instabilities in the oscillator can be caused by feedback from an external source and that feedback is also prevented by the Pockels cell isolator.

II.C. 2 Pockels Cell

The Pockels cells each consist of a single crystal of KDP placed between electrodes. They are Lasermetrics model 1071 and have a half wave voltage of 8 kilovolts (KV). The half wave voltage is the voltage required to change the crystal from a nonbirefringent plate to a half wave plate(47) for $1064 \mathrm{~nm}$. When no voltage is applied, the crystal behaves like a plate of glass, but only for light 
propagating in a single direction through the crystal. When the voltage is applied the index of refraction for light of one polarization is changed and the crystal becomes a half wave plate. For the correct orientation of the crystal, the half wave plate will rotate the polarization of linearly polarized incident light by 90 degrees. The complete configuration of the double Pockels cell switchout contains a $P$ polarizer followed by $a$ crystal and an $S$ polarizer. These elements are followed by a second crystal and a final $P$ polarizer. When the applied voltage is zero, the polarizers are crossed and a transmission of 1 part in $10^{5}$ is obtained. When the half wave voltage is applied, the crystals become half wave plates which rotate the polarization from $P$ to $S$ and then back to $P$ at the second crystal. The transmission of the system in the "on state" approaches unity. Thus the contrast of the isolation system is the ratio of the transmission of the "on state" to that of the "off state" and is $10^{5}$ for the double Pockels cell switchout system.

As mentioned before, the Pockels cell requires a voltage of $8 \mathrm{kV}$ in order to completely pass an incident $1064 \mathrm{~nm}$ pulse. Since the temporal separation of the incident pulses is $10 \mathrm{~ns}$, a high voltage pulse of $10 \mathrm{~ns}$ or less will select a single pulse. We want the selection process to be repeatable and so we require a high voltage switch that can deliver an approximately 10 ns electrical 
pulse with less than 1 ns of jitter between the electrical pulse and a trigger signal and do it at a repetition rate of $10 \mathrm{~Hz}$.

There are many possible methods to generate the required electrical pulse. We chose to use an EGG Rrytron model KN22B driven by an avalanche transistor stack. The high voltage switch can produce a $4 \mathrm{KV}$ pulse of any length from 1 ns to $I$ microsecond ( $\mu s)$ with a jitter of approximately $1 \mathrm{~ns}$. The $4 \mathrm{KV}$ voltage is half the optimum $8 \mathrm{KV}$ half wave voltage and causes the peak transmission of the double Pockels cell switchout to be only 25\%. The loss is unimportant as long as the seed pulse is above the noise level of the regenerative amplifier. Fortunately, the $4 \mathrm{KV}$ pulse and thus the Pockels cell transmission are stable to 18. One drawback of the krytron is that the internal delay between trigger and switching changes with age. The internal delay can range from 30 ns when the krytron is new to $60 \mathrm{~ns}$ when it is fully used. In addition, during an initial warmup period on any given day, the delay may change by as much as $5 \mathrm{~ns}$. The changing delay is compensated for by a variable delay adjustment in the trigger line using a BNC model 7030 delay generator. The electronics for generating the trigger pulse are described in Appendix 1. 
II.C. 3 Regenerative Amplifier

Once the single $1064 \mathrm{~nm}$ pulse is selected, it is injected into a regenerative amplifier (48) for amplification by a factor of $10^{5}$. The regenerative amplifier consists of a Nd:YAG amplifying head and an optical cavity. A seed pulse is injected into the cavity and passes through the amplifier head numerous times. We include within the cavity a single pockels cell of the same type as described above. The cell is in a double crystal configuration which reduces the half wave voltage to $4 \mathrm{kV}$ and allows another Krytron driver to be used. The Pockels cell provides a means for injecting and removing the optical pulse from the cavity as well as serving as a Q-switch for the cavity. In Q-switching, (49) the losses in the cavity are increased initially to prevent laser emission. Since laser action is prohibited, the population inversion in the YAG amplifier head grows to a much larger value than the normal threshold for lasing. The losses are then removed and the stored energy is released. A more complete description of Q-switching is given by Koechner. (49)

The chronology of operation for the regenerative amplifier is as follows. Three hundred microseconds before the $1064 \mathrm{~nm}$ pulse is selected for amplification, a trigger signal is sent to the YAG regenerative amplifier power supply which triggers the flashlamps. The excited state 
population inversion in the YAG rod is allowed to build up to its peak value which occurs soon after the peak of the flashlamp output. During the buildup time the Pockels cell in the regenerative amplifier cavity is in the "off state" so that the amplified spontaneous emission of the YAG rod is not allowed to be fed back by the cavity. When the inversion and hence the gain is at its peak, the single pulse is selected by the external double Pockels cell switchout.

The $1064 \mathrm{~nm}$ pulse is then injected into the regenerative amplifier cavity by reflecting off one of the polarizers in the cavity. The infrared pulse passes through the electro-optic crystal which is still in the "off state" and finally passes through the second polarizer which is aligned to pass light of the orthogonal polarization to the first polarizer. The pulse then passes through the amplifier head and is amplified. Next the pulse encounters the end mirror which has a $10 \mathrm{~m}$ radius of curvature. Upon reflection, it passes back through the amplifier head and is amplified again. Next it passes through polarizer number two. By this time, the voltage has been applied to the electro-optic crystal and the polarization of the infrared pulse is rotated by 90 degrees. The pulse now encounters the first polarizer which was used in reflection to inject the pulse, but since the pulse has had its polarization rotated it now passes through the polarizer and propagates 
to the second cavity end mirror, which is flat.

The $1064 \mathrm{~nm}$ pulse is now trapped within the cavity. Each time it encounters the electro-optic crystal its polarization is rotated by 90 degrees or "flipped". The pulse has one polarization in one half of the cavity and the orthogonal polarization in the other half. In addition, the infrared pulse passes through the amplifier head twice for every cavity round trip. After 22 round trips, much of the stored energy in the amplifier rod has been depleted and the growth of the pulse saturates. Since there are losses in the cavity due to all the elements, the amplitude of the $1064 \mathrm{~nm}$ pulse eventually decreases. We can monitor the evolution of the pulse by observing the amplitude of the pulse train that leaks out of the nearly $100 \%$ reflecting end mirror with a PIN diode. In Figure 2-2 we see one infrared pulse for every round trip in the cavity. The envelope on the pulse train is nearly gaussian due to the constant cavity losses and the gain saturation. For a stable system, the $1064 \mathrm{~nm}$ pulse will always reach its peak value a fixed time after entering the cavity. Typically, the peak of the train will fluctuate by only one pulse.

When the peak value is reached, the voltage to the electro-optic crystal is turned off. The switching is done when the infrared pulse is in the portion of the cavity which does not contain the amplifier head. The pulse passes 


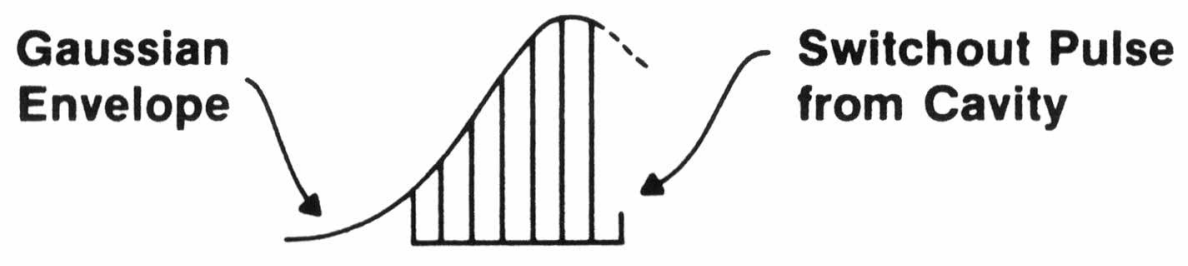

10 ns/Pulse

Figure 2-2 Regenerative amplifier pulse evolution 
through polarizer number one and then traverses the electro-optic crystal but is not rotated in polarization. When it encounters the second polarizer, it is rejected from the cavity.

\section{II.C.4 External Amplifier}

The rejected infrared pulse has an energy of $1 \mathrm{~mJ}$ and needs to be further amplified in an external amplifier. In the regenerative amplifier the size of the beam is restricted to be only a few millimeters. The restriction is imposed once a cavity configuration is chosen which is both a stable resonator and contains no lenses. (50) Because of the small beam size, the entire area of the amplifier rod cannot be accessed. In addition, the $1 \mathrm{~mJ}$ beam is approaching the damage threshold for YAG ${ }^{(51)}$ and it is wise to further amplify the $1064 \mathrm{~nm}$ pulse external to the regenerative amplifier where the beam cross section can be enlarged.

The external amplifier is a Quantel (SF 410-07) laser head with a $7 \mathrm{~mm}$ by $115 \mathrm{~mm}$ rod and is used in a double pass configuration. We prefer to use the largest beam size possible in order to extract the largest amount of energy and avoid damage problems. We have chosen to separate the input and output beams to the amplifier not by spatial means but by polarization in order to obtain the largest diameter beam. The input beam is set to be approximately $4 \mathrm{~mm}$ in 
diameter by a collimating lens placed after the regenerative amplifier. Four millimeters is the largest beam diameter possible without causing a large amount of degradation in the beam profile due to diffraction from the edge of the rod. (52) The beam is then directed through the amplifier rod after reflecting off a thin film polarizer. After amplification, the beam passes through a quarter wave plate and becomes circularly polarized. It is then autocollimated by a $100 \%$ reflecting mirror. It traverses the quarter wave plate again and now has one half wave retardation. As discussed above, the half wave of retardation rotates the polarization of the beam by 90 degrees from when it was in the amplifier before. After passing through the amplifier the second time it is then transmitted through the injection polarizer and finally separated from the input beam. The $100 \mathrm{ps}, 1064 \mathrm{~nm}$ infrared pulse now contains $30 \mathrm{~mJ}$ of energy.

II.C.5 Frequency Doubling

To excite the dye gain medium in the dye amplifiers, we require a pump pulse with a wavelength near $500 \mathrm{~nm}$. Thus, the next step is to frequency double the $1064 \mathrm{~nm}$ pulse. There are many crystals which can be used. We have chosen $C D A$ and $C D * A$ because of their high doubling efficiency. Both are temperature tuned crystals with phase matching temperatures of $42^{\circ} \mathrm{C}$ and $102^{\circ} \mathrm{C}$ respectively. (53) An error in the temperature can be partially offset by adjusting the 
crystal angle away from normal. To align the doubling crystal correctly the temperature oven is set to a temperature a few degrees below the phase matching temperature. The crystal is then tilted through an angle of a few degrees from the normal in each of two directions perpendicular to the direction of propagation of the $1064 \mathrm{~nm}$ beam. In one direction, the doubling efficiency is not very sensitive to the angle but in the other direction the doubling efficiency varies greatly.

A plot of doubling efficiency versus angle is shown in Figure 2-3. The data is for $C D^{*} \mathrm{~A}$ at $1054 \mathrm{~nm}$ but it demonstrates the important features. For a temperature below the optimum, a distribution with two equal lobes and a minimum at normal incidence is observed. As the temperature is increased, the two lobes of the curve move together and at the optimum temperature, Topt' they join. Using this technique the angle is aligned and the temperature adjusted. Finally, the crystal is rotated about the direction of propagation in order to optimize for a given polarization.

Theoretically, $100 \%$ of the incident light can be converted to the second harmonic. The first reduction in the ideal value is due to losses by reflection and absorption. The windows of the cell containing the crystal have antireflection coatings. The entrance window is antireflection coated for the fundamental wavelength and the 


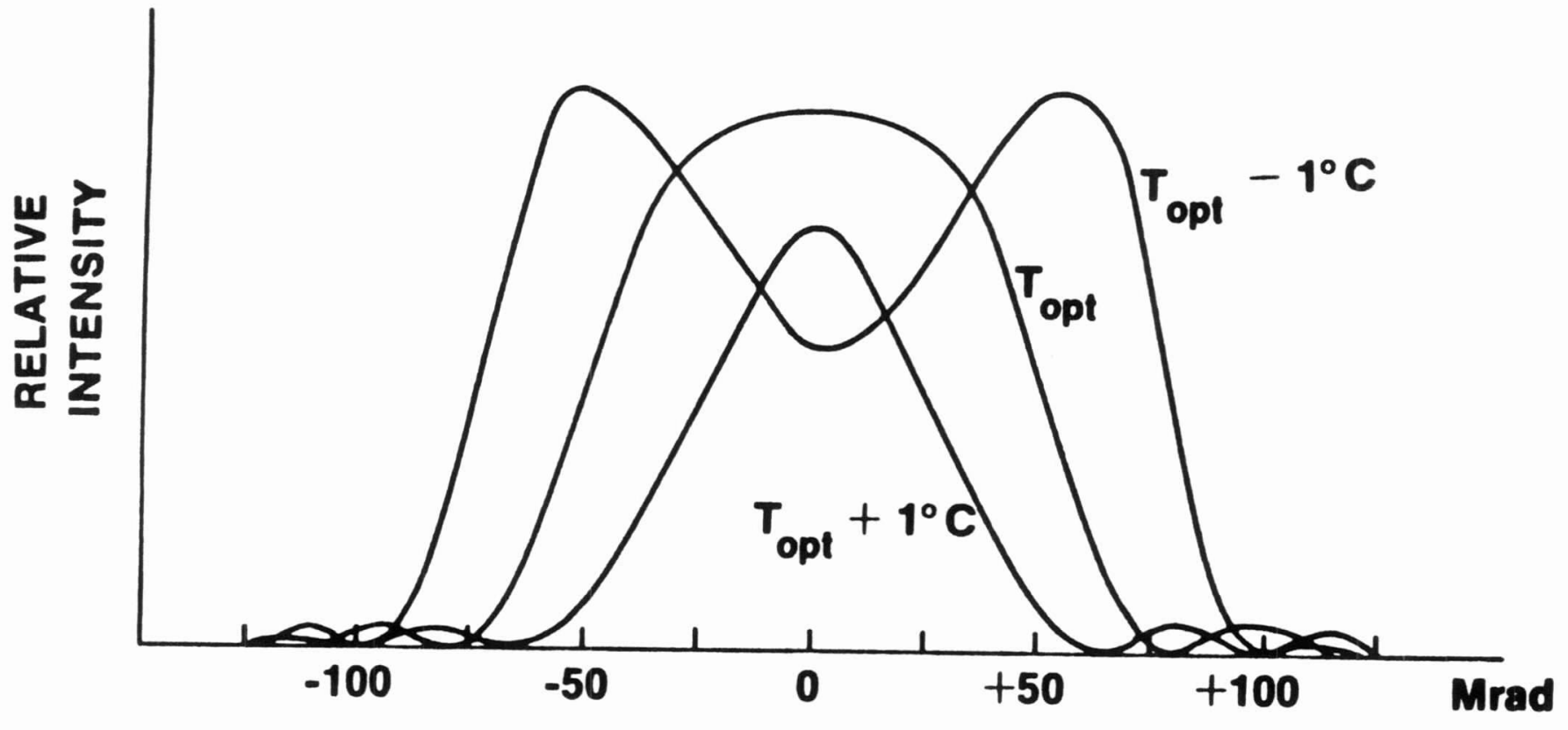

Figure 2-3 Angular dependence of frequency doubling 
exit window is coated for the second harmonic. In addition the crystal is surrounded by an index matching fluid. The $C D * A$ crystal has very small losses due to absorption; however, the losses are not negligible for the CDA crystal. The overall loss for the $C D * A$ assembly is approximately 58 while the CDA assembly exhibited a loss of 25\%. The ratio of second harmonic to fundamental after passing through the crystal is at best 3:2 for both of the crystals used. Since the $C D * A$ has less loss it is the preferable crystal with the exception that it becomes chemically unstable near its phase matching temperature. Because of this practical problem, most experiments were performed with CDA.

There are also properties of the infrared laser pulse which can prevent complete conversion to the second harmonic. (54) Shown in Figure $2-4$ is the ideal curve for doubling efficiency (55) as a function of input intensity. If the infrared pulse could be generated as a square pulse in both space and time, then complete conversion could be achieved. Typically, however, the $1064 \mathrm{~nm}$ pulse is gaussian in space and time and therefore the wings of the pulse do not double as efficiently. Any further variation on the temporal or spatial pulse shape will in general decrease the doubling efficiency further.

For an infrared pulse of $30 \mathrm{~mJ}$ in $100 \mathrm{ps}$ incident on the doubling crystal, we typically receive $10 \mathrm{~mJ}$ of infrared 


\section{CONVERSION EFFICIENCY (\%)}

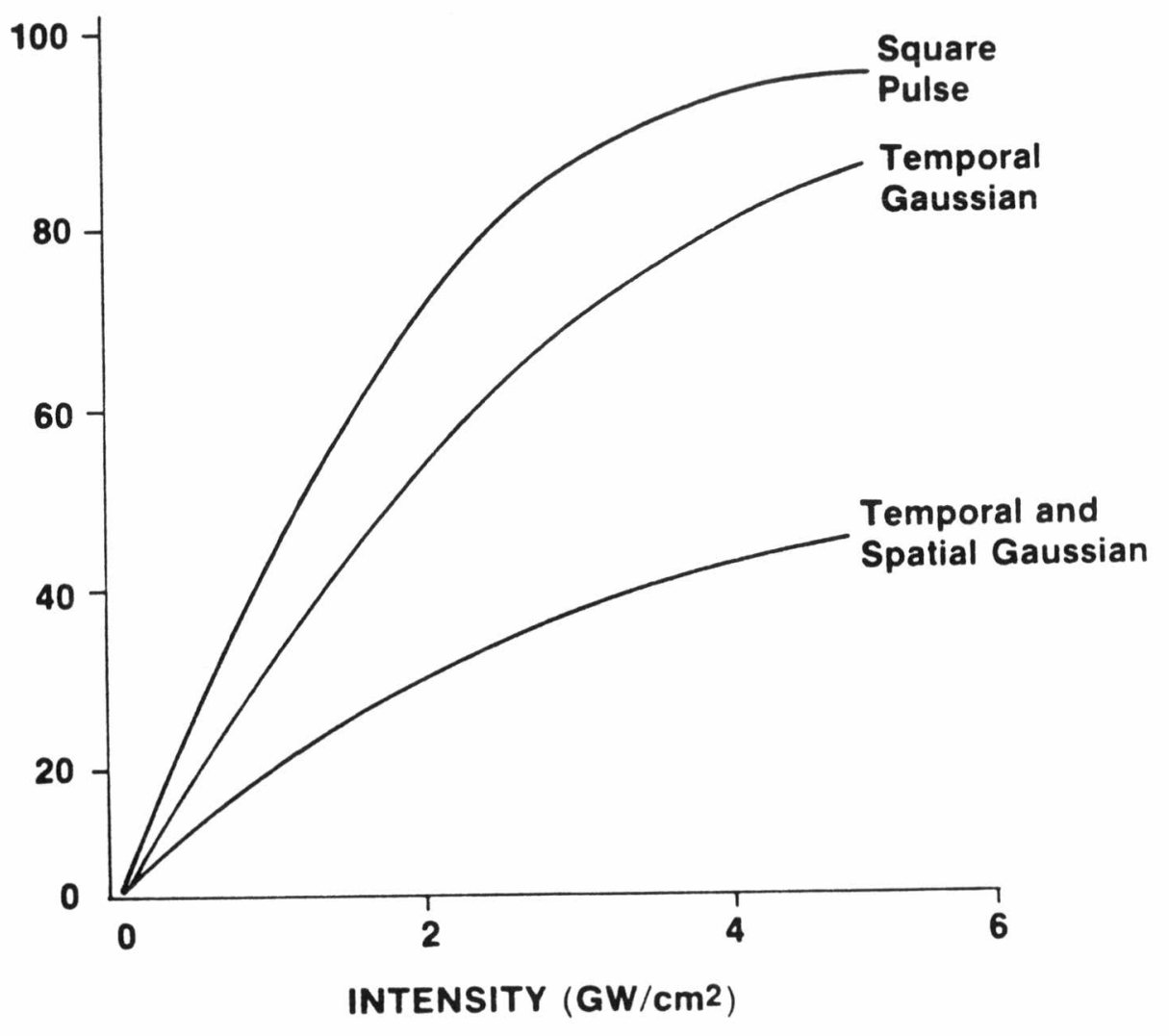

Figure 2-4 Intensity dependence of frequency doubling 
and $10 \mathrm{~mJ}$ of the second harmonic. The green pulse has a duration of $70 \mathrm{ps}$ and is the pump pulse for the dye amplifiers. Since the green pulse has been derived from the same source used to pump the dye oscillator, it is well synchronized to the subpicosecond pulses generated by the dye oscillator, as will be shown next.

\section{II.C. 6 Temporal Jitter}

Three major sources of jitter between the input dye pulses and the frequency doubled $532 \mathrm{~nm}$ pump pulses can be identified. In decreasing order of importance they are: 1) the jitter in the process of synchronous pumping, 2) the temporal reshaping of the pump pulse inside the regenerative amplifier, and 3) the variation in the path lengths that the two pulses must travel.

In the synchronously pumped oscillator, each green pulse will excite the dye medium. The gain increases during the green pump pulse as shown in Figure 2-5, until the gain becomes greater than the 1oss. It is at this point in time that the dye pulse can form or can be amplified. (42) The arrival time of the green pulse is tied to the precision of the Rockland synthesizer which is accurate to one part in $10^{7}$. For a dye pulse separation of $10 \mathrm{~ns}$, this accuracy implies a jitter of only a few femtoseconds. The amplitude of the green pulse, however, can fluctuate causing the time when the gain exceeds the loss to fluctuate correspondingly. 

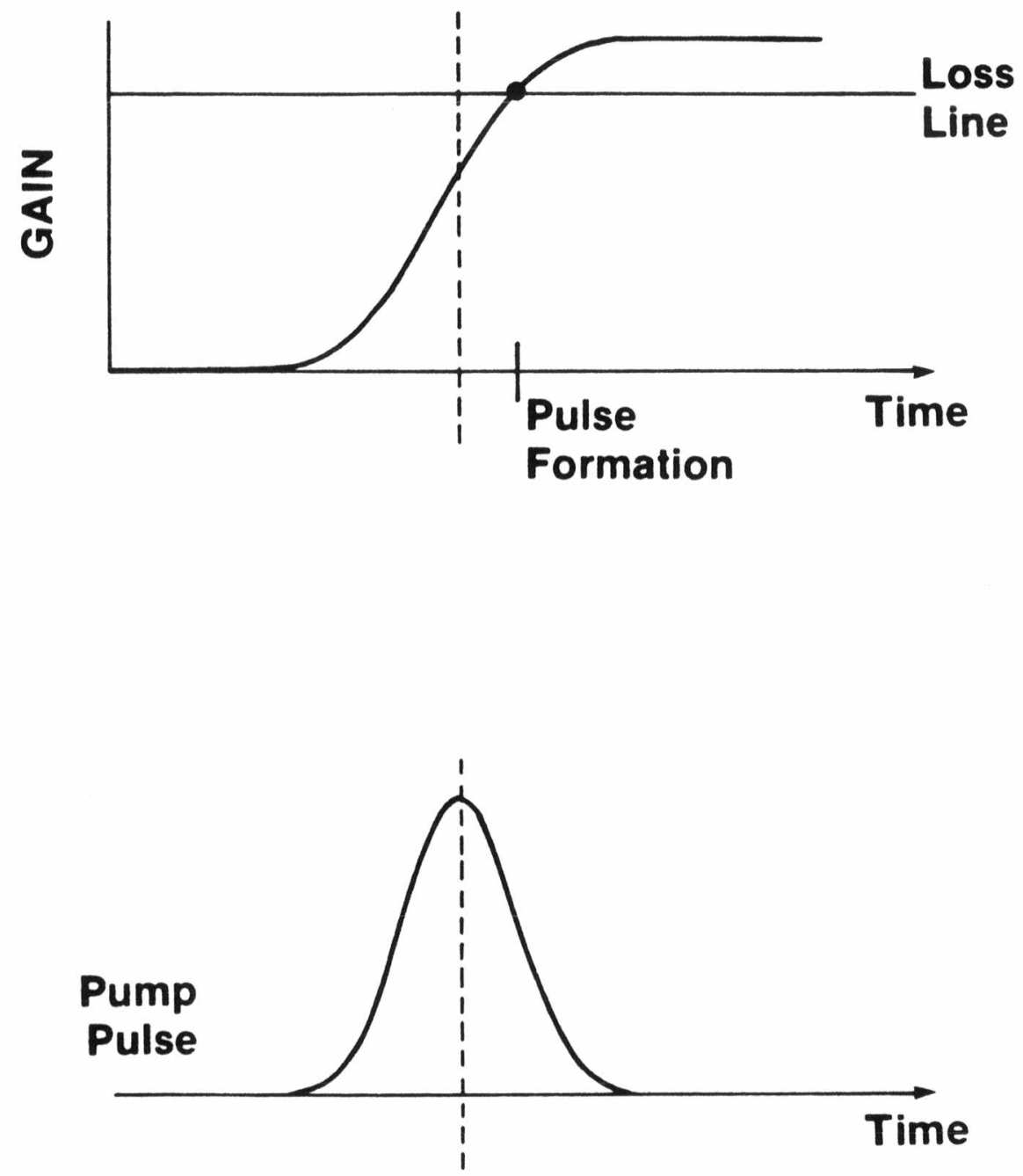

Figure 2-5 Oscillator pulse formation 
For a 58 intensity fluctuation in the green pulse and a laser operating at 1.2 times the lasing threshold, the calculated jitter is $3.85 \mathrm{ps}$.

A second source of jitter is the reshaping that the infrared pulse undergoes while being amplified in the regenerative amplifier. Since the $1064 \mathrm{~nm}$ pulse is amplified in the saturated regime, the leading edge of the pulse will be preferentially amplified and the pulse will appear to travel at a velocity greater than the speed of light in the YAG amplifier. If the amplitude of the infrared pulse out of the regenerative amplifier fluctuates by 208 in a train of 10 pulses, we estimate a temporal jitter of $200 \mathrm{fs}$.

Finally, the two pulses travel through long paths of air which have different lengths. The infrared pulse must pass through 250 ns or 75 meters (m) more air than the pulse from the dye oscillator. If we assume a $2^{\circ} \mathrm{C}$ fluctuation in temperature throughout the laboratory at any time, we find that the index change in the air can cause a temporal shift of $200 \mathrm{fs}$.

Combining these three jitter sources we obtain an estimate of less than 5 ps for the jitter between the dye input and the doubled YAG pump pulses for the optically synchronized system. Experiments, which will be presented in Chapter III, put an upper limit of $30 \mathrm{ps}$ on the jitter 
between the input pulse from the dye oscillator, and the pump pulse from the regenerative YAG amplifier.

II.C. 7 RF Coupled System

A second system for preparing the synchronized pump pulse has been constructed and is called the RF coupled amplifier system. (56) Two modelocked lasers can be synchronized by using the same $R F$ signal to drive the acousto-optic modelockers in each cavity. Previous work by Harvey, Gabel, and Mourou(57) showed that a modelocked Argon laser and a Q-switched modelocked YAG laser can be synchronized to $18 \mathrm{ps}$. In Chapter III, it will be shown that the jitter between the pump and input pulses need only be less than 100 ps for efficient amplification.

We have chosen the RF coupled technique to couple the CW modelocked laser that is used to pump the dye oscillator to a second $\mathrm{CW}$ modelocked laser. The second laser is also a Quantroniz Model 114 and is the same as the one described earlier with the important difference that it is also Q-switched. The maximum repetition rate for the Q-switching is $1.6 \mathrm{kilohertz}(\mathrm{KHz})$. The sequence of events during one Q-switch cycle is as follows. Initially, the acousto-optic Q-switch places a loss in the cavity so that the laser is running just above threshold. A low energy $1064 \mathrm{~nm}$ pulse is then allowed to form by repeatedly passing through the modelocker. This condition, known as the prelase, (58) helps 
establish the synchronization between the two YAG lasers by providing a longer buildup time for the infrared pulse and subsequently a greater number of trips through the modelocker.

After the prelase has been established, the Q-switcher removes the loss from the cavity and the $1064 \mathrm{~nm}$ pulse is allowed to grow. The RF signal for the Q-switcher is derived from the same frequency synthesizer that drives the two modelockers and the Q-Switch is gated on a zero crossing of the carrier frequency for better stability. The output of the laser is a train of 25 pulses with an amplitude stability of plus or minus 18. A krytron driven pockels cell, as described previously, is used to select a single pulse which has an energy of $25 \mu \mathrm{J}$. The system can be used at low energy with a repetition rate of $1.6 \mathrm{KHz}$ or the repetition rate can be decreased to $10 \mathrm{~Hz}$ and the pulse amplified. For additional amplification, the selected pulse is passed through a series of three single pass YAG amplifiers which increase the $1064 \mathrm{~nm}$ pulse energy to the $30 \mathrm{~mJ}$ level. The pulse is then frequency doubled and becomes the pump pulse for the dye amplifiers.

II.C. 8 Comparison of Systems

The two pump pulse preparation systems are shown in block form in Figure 2-6. The RF coupled system has several advantages over the optically coupled system. First and 
I

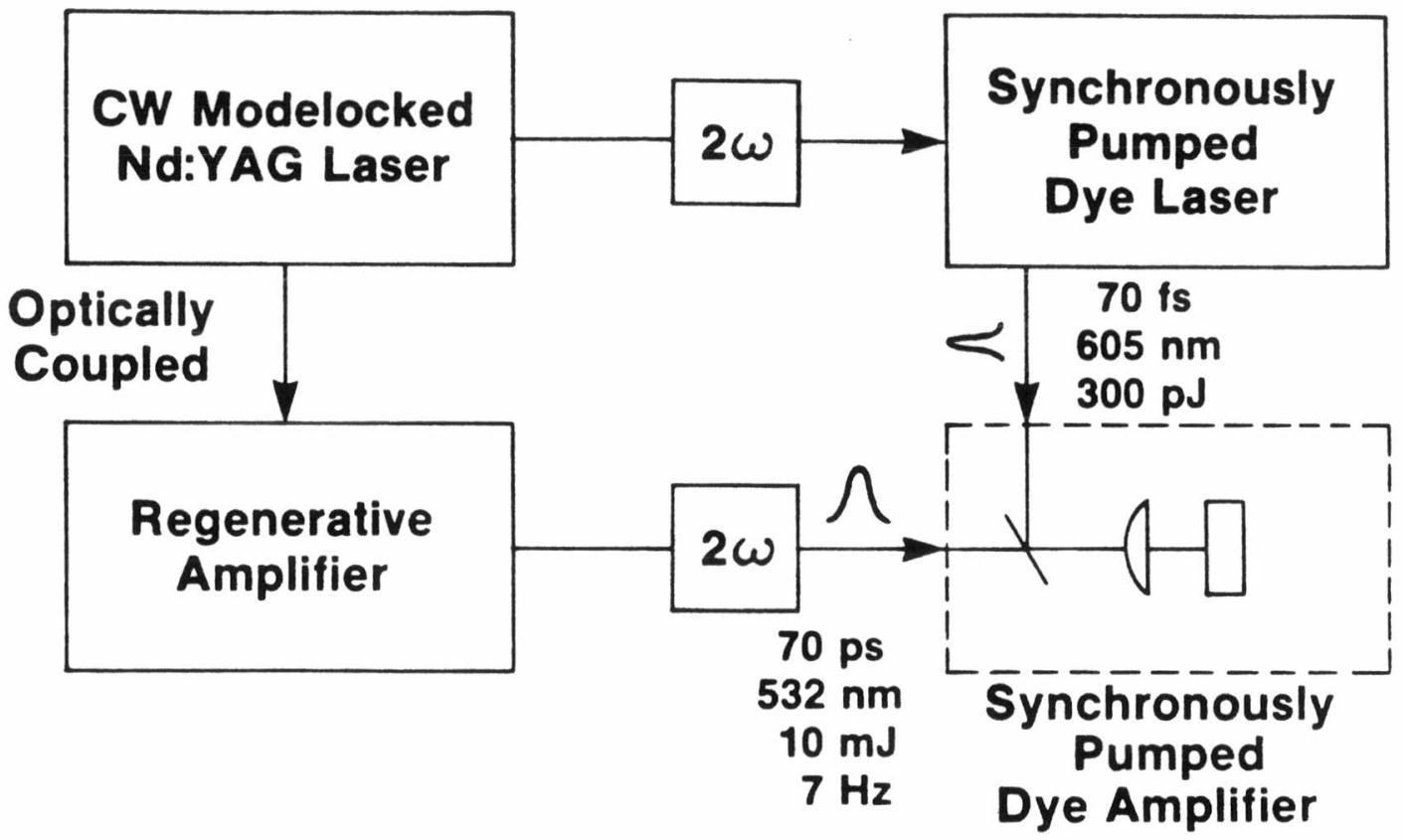

II

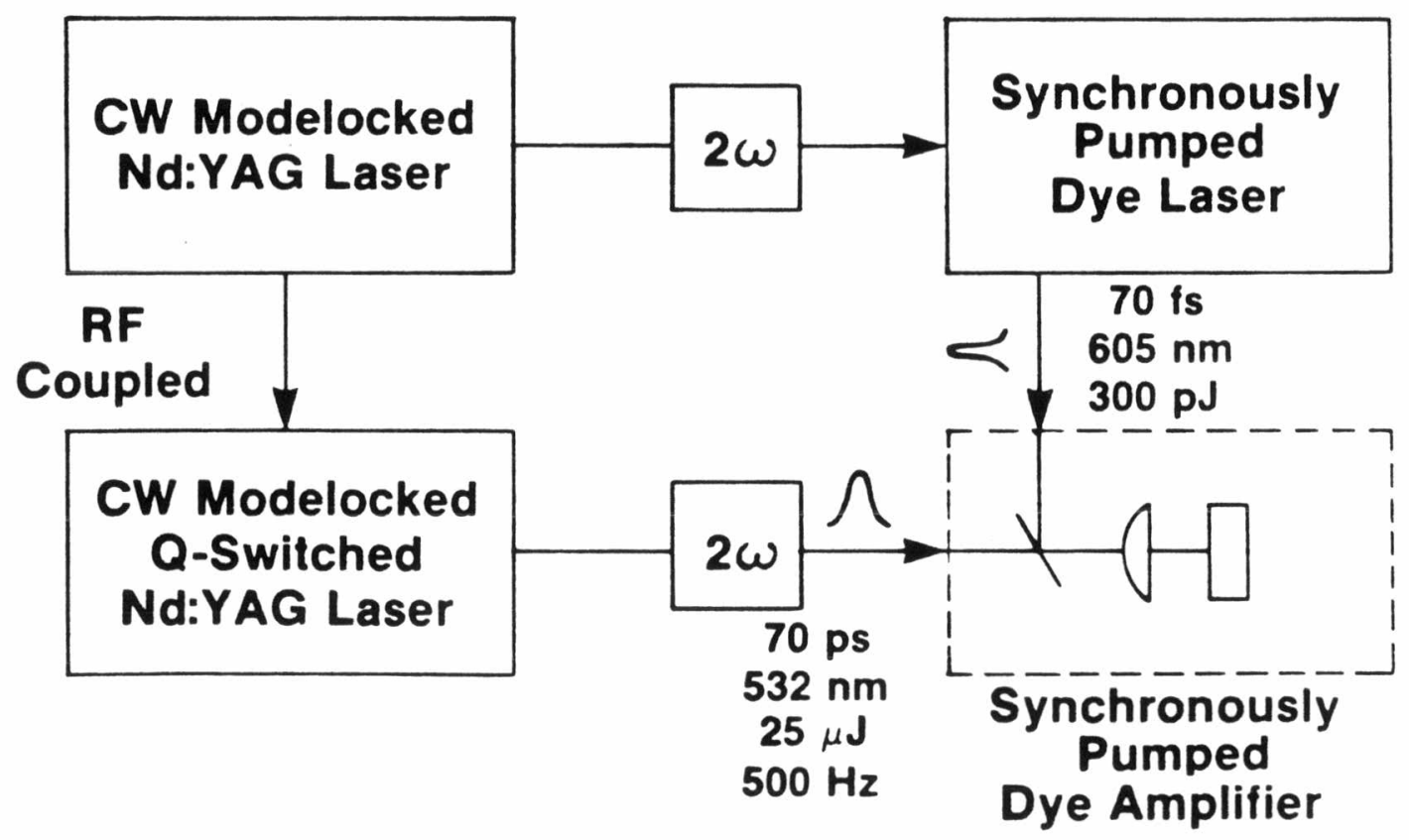

Figure 2-6 Comparison of synchronous amplifier systems 
foremost is the ability to run at high repetition rates. The limiting factor for the repetition rate is the ability to establish the prelase that is required for stability and synchronization. When the Q-switch first inserts the loss in the cavity, relaxation oscillations occur which must damp out to establish the prelase. The damping time of 2 milliseconds (ms) limits the repetition rate to 500 Hertz (Hz). If a feedback loop which monitors these oscillations is used to drive the Q-switch, the oscillations can be damped more quickly allowing a repetition rate of $1.6 \mathrm{KHz}$. A high repetition rate system is advantageous because it allows for $\mathrm{CW}$ alignment of the components that follow and the ability to perform high repetition rate amplification. By using the RF coupled oscillator laser and a CD*A crystal but with no additional YAG amplifiers, Duling(34) was able to amplify a picoseond pulse to $350 \mathrm{~nJ}$ at a repetition rate of $500 \mathrm{~Hz}$.

In addition to the convenience of using $\mathrm{CW}$ alignment proceedures, the RF coupled laser optically decouples the oscillator and amplifier systems. Since it is no longer necessary to mode match the $C W$ YAG into a regenerative amplifier, initial alignment of the system is greatly facilitated. Finally, the RF coupled system requires only one Krytron to drive the Pockels cell as opposed to the optically coupled system which required two. Removal of a Krytron has improved both the short term stability and the 
long term reliability of the system. Overall, the RF coupled laser provides a more versatile system for pumping dye amplifiers than does the optically coupled system.

\section{II.C.9 Temporal Jitter}

The only disadvantage of the RF coupled system is that the jitter between the two YAG lasers is greater than in the optically coupled case. A good estimate for the jitter in the optically coupled system was found to be $5 \mathrm{ps}$. To estimate the jitter for the RF coupled system is more difficult. Short term jitter can be caused by noise on the RF signal or fluctuations in the cavity gain. The main source of long term fluctuations is the differing response of the two modelockers to the same $R F$ input signal. The modelocker crystal is made of quartz and is a resonant device. Changes in the ambient temperature can change the resonant frequency of the modelockers. The detuning from resonance changes the quantity of RF power absorbed and thus the modulation depth causing a phase shift in the infrared pulse train. (57)

A measurement of the jitter can be found experimentally. Using the zero background autocorrelation technique, (28) we can measure the pulsewidth of each one of the YAG lasers. Using the same technique the cross correlation between the two lasers can be measured. Assuming a gaussian distribution, the width of the cross 
correlation is due to the widths of the individual $1064 \mathrm{~nm}$ pulses and the jitter between the two pulses as given in the following equation.

$$
T_{\text {cross correlation }}^{2}=T_{1}^{2}+T_{2}^{2}+T_{j i t t e r}^{2}
$$

Using Equation 2-1, a value of 40 ps was assigned to the jitter. (34) During dye amplification, the long term drift (30 minutes) was found to be $200 \mathrm{ps}$.

In summary, the optically coupled system for preparing a synchronous pump pulse provides better synchronization while the RF coupled system features higher repetition rates and ease of alignment. 


\section{I.D Dye Amplifiers}

In this section I will describe the design and operation of the subpicosecond dye amplifier system. I have described above the procedures for preparing an ultrashort dye input pulse and a synchronized frequency doubled $532 \mathrm{~nm}$ pump pulse. Given the temporal width, energy, polarization and wavelengths of the input and pump pulses, the object is to design the optimal amplifier system.

In the design portion I will discuss the parameters which can be varied in order to optimize the amplification system with respect to efficiency and the preservation of the temporal pulse shape. The reasons for a multiple stage system will be explained and the scaling from one stage to the next discussed. Next, I will deal with the choice of dyes for the gain medium and the issue of timing the amplifier.

The subpicosecond dye amplifier system that has been constructed is then described. The performance of the amplifier system is analyzed with respect to gain and pulse width. The important topics of nonlinear effects and ASE suppression are then discussed. 


\section{II.D. I Parameters}

We wish to construct an amplifier system that produces an ultrashort high energy pulse. The amplifier should have good beam quality, low ASE background and high efficiency as discussed in section II.A. To optimize any amplification system, the parameters that can be varied must first be identified. The input pulse has a wavelength, energy, polarization, duration and temporal shape. The pump pulse possess all of these parameters plus a relative time of arrival with respect to the input pulse. In the dye amplifier itself the parameter space includes: choice of a particular dye as gain medium, concentration of dye, choice of solvent, amplifier length, spot size of the input pulse in the amplifier and a choice of pumping geometries.

Some of the optical parameters have been fixed previously by our selection of dye input wavelength and green pump pulse preparation systems. The wavelength, energy, duration and pulse shape of the pump and input pulses are fixed by restriction on the lasers that prepared these pulses. The polarization of the pump and input pulses can be changed by the insertion of a waveplate. An optical or electrical delay line will be used to vary the relative delay between the pump and input pulses. This arrangement decouples the delay from the alignment of the amplifier; the delay will turn out to be a crucial parameter in the 
optimization of the system.

I will now consider the material parameters. In the dye amplifier, the choice of solvent is based on nonlinear index considerations. The choice of dye is dictated by the wavelength of the input pulse. The amplifier length and concentration can be largely traded off against one another and so I have chosen to fix the amplifier length at a convenient value and leave the dye concentration, which is simple to vary, as a free parameter. A pumping geometry was chosen based on timing considerations and beam quality. Finally, the spot size of the input pulse in the amplifier is intimately related to both amplifier efficiency and distortion of the temporal pulse shape. As such, an approximate value for the spot size was calculated and implemented with the freedom to vary it somewhat about the set value being built into the system.

\section{II.D. 2 staging}

The first question that must be addressed is how many stages are required. Certainly, only one stage would greatly simplify the system. The target for the system is to increase a subpicosecond pulse with $100 \mathrm{pJ}$ of energy to the millijoule level which requires a gain of $10^{7}$. There are two major processes that put an upper limit on the gain. For typical input pulse intensities and an amplifier with a roughly cylindrical active area, saturation will limit the 
gain to $10^{5}$ (see section IV.D.1). A small input pulse incident on the front of the amplifier will grow exponentially in energy at first until it begins to deplete the stored energy in the amplifier. At this point the gain begins to saturate and the pulse grows more slowly. Thus, without radically changing the shape of the active region, saturation will limit the gain.

A second factor which can limit the gain is amplified spontaneous emission (ASE). The quantity of ASE produced by an amplifier depends strongly on the gain. The strong dependence results because a higher gain amplifier also has a greater amount of spontaneous emission with which to start the ASE. As the gain of the amplifier is increased, the ASE will increase faster. As a result, a limit will occur after which more excited molecules will lead to more ASE but little more gain. The gain limit depends on the relative rates of pumping the amplifier and the depletion of energy due to ASE. The problem is treated theoretically in section IV.F.2.

One other factor that can limit the gain is excited state absorption. When most of the molecules in the dye are excited from the ground state $s_{0}$ to the first excited state $\mathrm{S}_{1}$ it is possible to pump these molecules to a higher lying excited state $S_{2}$. The molecules in $S_{2}$ relax quickly to $S_{1}$ and are then available for amplification; however, a pump 
photon has gone into heat and has been wasted in the process. The conclusion, then, is that more pump photons may not mean more excited molecules and thus the gain will be limited.

From the above considerations, it appears that more than one stage is required. Many stages could be used and each stage could be tailored to avoid saturation by enlarging the active region. In addition, the amount of ASE would be small since the gain in each stage would be small. Adding an extra stage, however, adds additional losses to the system by increasing the number of optical components in the beam. In addition, the complexity and cost of a many stage system becomes prohibitive. We have compromised and have chosen to build a three stage system. Three stages are both necessary and sufficient to reach the millijoule output energy level without pulse broadening.

At this point, we can determine some of the parameters for the three stages. We have chosen to use longitudinal pumping in order to preserve the timing and have a better beam quality as described in Section II.D.7. Moreover, we have decided upon $1 \mathrm{~cm}$ amplifier lengths for all three stages. One centimeter is shorter than the Rayleigh range for the pump and input beams in all three stages. The Rayleigh range (59) is the distance from focus that a gaussian beam will propagate before its cross sectional area 
increases by a factor of two. In addition a $1 \mathrm{~cm}$ cell is long enough to avoid such high dye concentrations that dimer formation becomes a problem. For convenience, the choice of solvent will be the same throughout the system. Given the above decisions, the choice of dye will also be the same in each stage. The choice of using the same dye in each stage is not an obvious one and depends somewhat on the pumping geometry, as will be explained in section II.D.7. The concentration of the dye is optimized experimentally as is the size of the active region in each amplifer. Approximate spot sizes for each amplifier stage are calculated in Section II.D. 12 .

\section{II.D. 3 Saturation}

Saturation of the input pulse is a process which presents a trade off. To build an efficient amplifier system, it is desirable to remove as much of the stored energy from the amplifier as possible and transfer it to the input pulse. This is a description of the saturated gain regime. In addition, if the input amplitude should fluctuate, the output will not change greatly since the output depends more strongly on the amount of energy stored. Thus in order to have a stable and efficient amplifier, it is advantageous to operate far into saturation.

The trade off results because, when the amplifier is run in saturation, the temporal shape of the input pulse 
will not be preserved. In particular, the higher intensity spatial portions of the input pulse will be amplified less than the rest of the pulse. Spatially, saturation is helpful, since it tends to smooth out the beam profile. Temporally, however, saturation translates into a broadening of the pulsewidth. The result of amplifying a subpicosecond pulse well above the saturation level can be severe and the pulse broadening unacceptable in a subpicosecond system. Theoretical predictions of the pulse broadening are presented in section IV.G. A compromise must be reached between the helpful and deleterious effects of saturation. A good solution is to design each amplifier so that the input pulse reaches the saturation level at the output face of the stage. This solution guarantees a minimum of temporal distortion while providing a relatively efficient system. Saturation is investigated experimentally in Section III.F.

The intensity of the input beam can be adjusted by varying the spot size in the amplifier. If the energy per unit area of the input is equal to the saturation flux, half of the energy stored in the amplifier will be removed and the amplifier is said to be saturated. The value of the saturation flux depends on the dye used and for rhodamine $6 \mathrm{G}$ it is $1.4 \mathrm{~mJ} / \mathrm{cm}^{2}$. Previous three stage systems have had gains of $10^{3}, 10^{2}$ and 10 respectively for each stage. (35) If we assume that for some value of the pump energy we can 
obtain the same gains, then we can estimate the spot sizes required for each stage. With an input of $100 \mathrm{pJ}$, a spot size of $50 \mu \mathrm{m}$ will allow the input pulse to just reach the saturation flux at the back of the first stage. The corresponding spot sizes for the second and third stages are $500 \mu \mathrm{m}$ and $1.5 \mathrm{~mm}$ respectively. The trend is true for any multistage amplifier system; the later stages will have larger spot sizes. Since the output intensity increases in the later stages, the larger spot sizes are required to keep the successive stages at the saturation level.

II.D. 4 Baseline Design

In summary, the baseline design includes three amplifiers consisting of $1 \mathrm{~cm}$ cells filled with the same dye and solvent. The geometry for pumping is longitudinal for all three stages. We leave the concentration of the dye and the exact spot size as free parameters to be optimized during use; however, the trend will be for the spot size to increase in the later stages. Based on these principles, an amplifier system was built as shown in Figure 2-7.

II.D. 5 Dye

The dye oscillator produces the shortest pulses at $605 \mathrm{~nm}$ as described in section II.B.6. The following dyes were all tried for amplification of the $605 \mathrm{~nm}$ light: rhodamine 590 (rhodamine 6G), rhodamine 610 (rhodamine B), 


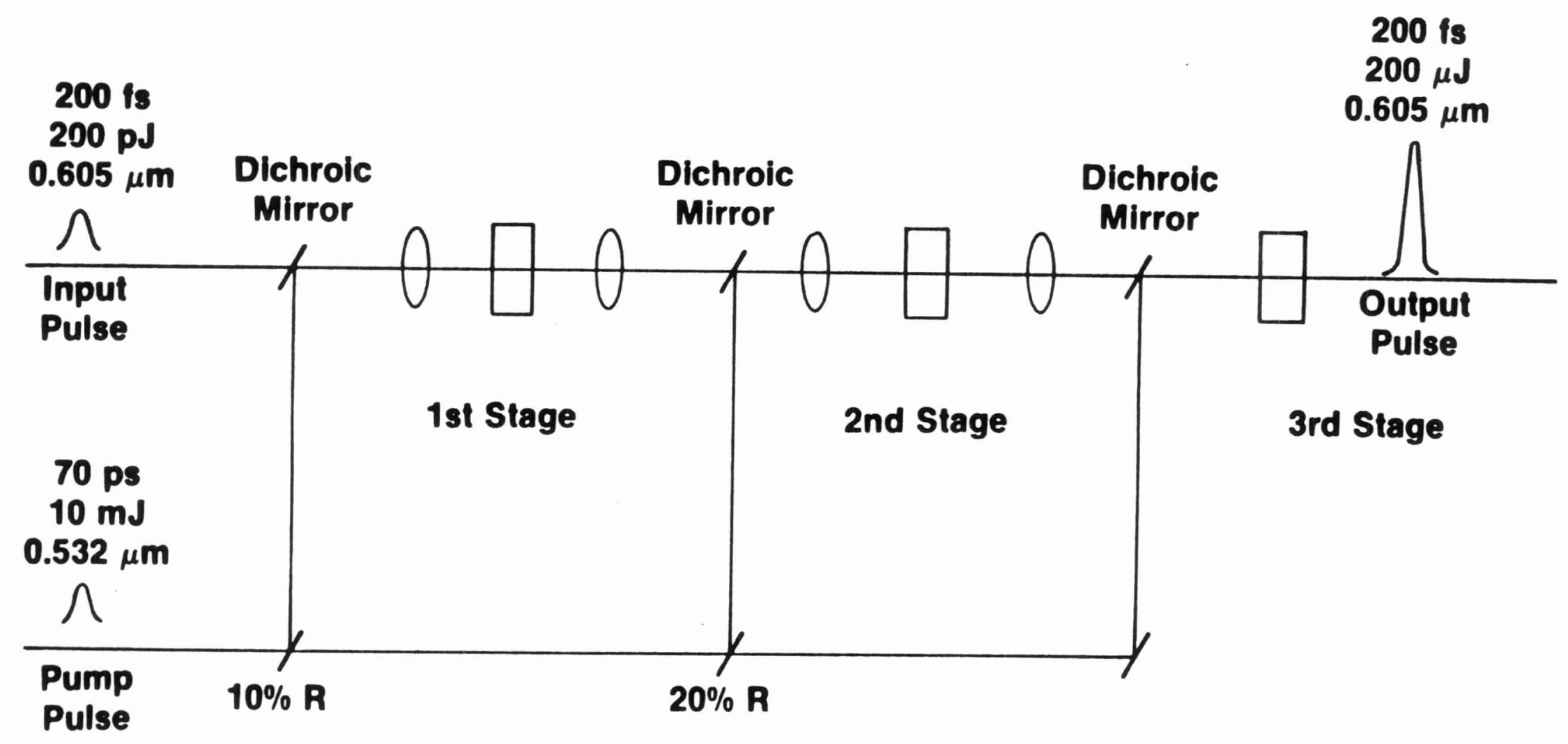

Figure 2-7 Three stage dye amplifier system 
kiton red 620, rhodamine 640, sulforhodamine 640 , cresyl violet and DCM. All dyes were tried in equal concentrations and in the same geometry. Easily the best dye was found to be kiton red. The superiority of kiton red is not surprising once we examine the absorption and emission characteristics of the dye. The ideal dye should have a peak absorption at $532 \mathrm{~nm}$ and peak emission at $605 \mathrm{~nm}$. Unfortunately, all the dyes in the above list (with the exception of DCM) have a stokes shift or separation of their absorption and emission peaks of only $60 \mathrm{~nm} .^{(60)}$ we are faced with the trade off between decreased absorption or decreased emission cross sections. Probably the best dye would have its peak emission just on the yellow side of $605 \mathrm{~nm}$. Such an emission peak would allow a slightly larger absorption cross section at $532 \mathrm{~nm}$ while decreasing the reabsorption at $605 \mathrm{~nm}$. Kiton red meets the above criteria with its peak emission at $595 \mathrm{~nm}$. (The peak is measured at $10^{-4} \mathrm{M}$ dye concentration. At lower concentrations the peak moves to $575 \mathrm{~nm}$ because of lower reabsorption).

At other times, the dye oscillator was run at different wavelengths. When the input pulse was at $590 \mathrm{~nm}$, the optimum dye for the amplifiers was found to be rhodamine 6G. The optimum dye for use at $615 \mathrm{~nm}$ was found to be sulforhodamine 640 although it was not as efficient as rhodamine $6 \mathrm{G}$ was at $590 \mathrm{~nm}$. The remainder of the work described in this thesis was done at $605 \mathrm{~nm}$ and with the dye 
kiton red.

II.D. 6 Solvent

A few of the solvents used by previous workers include: water, ethanol, methanol, ethylene glycol, and DMSO. The choice of a solvent is based primarily on its nonlinear properties and its ease of use. Other properties of lesser interest are the interaction between the solvent and the dye which can cause triplet quenching, shifts of the absorption and emission bands, and damping of molecular rotation.

We experimented with ethylene glycol, methanol and water. The solvent was placed without any dye in a $1 \mathrm{~cm}$ flowing cell and pumped with a high intensity beam. The output was observed with color filters and Raman emission in the red and blue were observed. The solvent with the highest threshold for Raman emission was found to be water. A large f-number beam was used for these tests and it is assumed that self focussing of the beam due to nonlinear index effects will enhance the Raman emission. The amount of beam distortion of the Raman emission, however, was not severe. Water is the most convenient solvent to use when changing dye solutions frequently. Ethylene glycol is hard to flush out of the flow system and DMSO requires care in handling. Methanol and ethanol are convenient but evaporate more quickly than water causing a change in the concentration of the dye. Other researchers ${ }^{(35)}$ have used 
water with a small quantity of soap (28 Ammonix L.O.) mixed in to quench triplet states and prevent the formation of dimers. We observed no improvement with the addition of soap. A null result was anticipated since we excite the amplifiers in $70 \mathrm{ps}$ and the decay rate into the triplet state is on the order of $2 \mu \mathrm{s}$. (61) In addition, our concentration $\left(10^{-4} \mathrm{M}\right)$ is below the concentration where dimer formation becomes a problem. (62)

The amplifiers consist of 1 cm flowing cells type 501 from Precision cells. The cells are oriented at Brewsters angle yielding an active length of $1.3 \mathrm{~cm}$. The flow systems consist of a Micropump pump and a l liter reservoir which can be sealed. The cells are attached using soft Tygon tubing to damp out any oscillations from the pump. The reservoir is filled sufficiently to cover the input hose. Covering the hose prevents the formation of bubbles in the system. Water has less severe bubble problems than ethylene glycol.

II.D.7 Pumping Geometry and Beam Quality

There are two major classes of pump geometries shown in Figure 2-8: longitudinal and transverse. The advantage of transverse pumping is that it is simpler. Since the pump beam does not need to be combined with the input, no costly dichroic mirrors are needed. More importantly, the two beams are not coupled and the spot size of one may be 

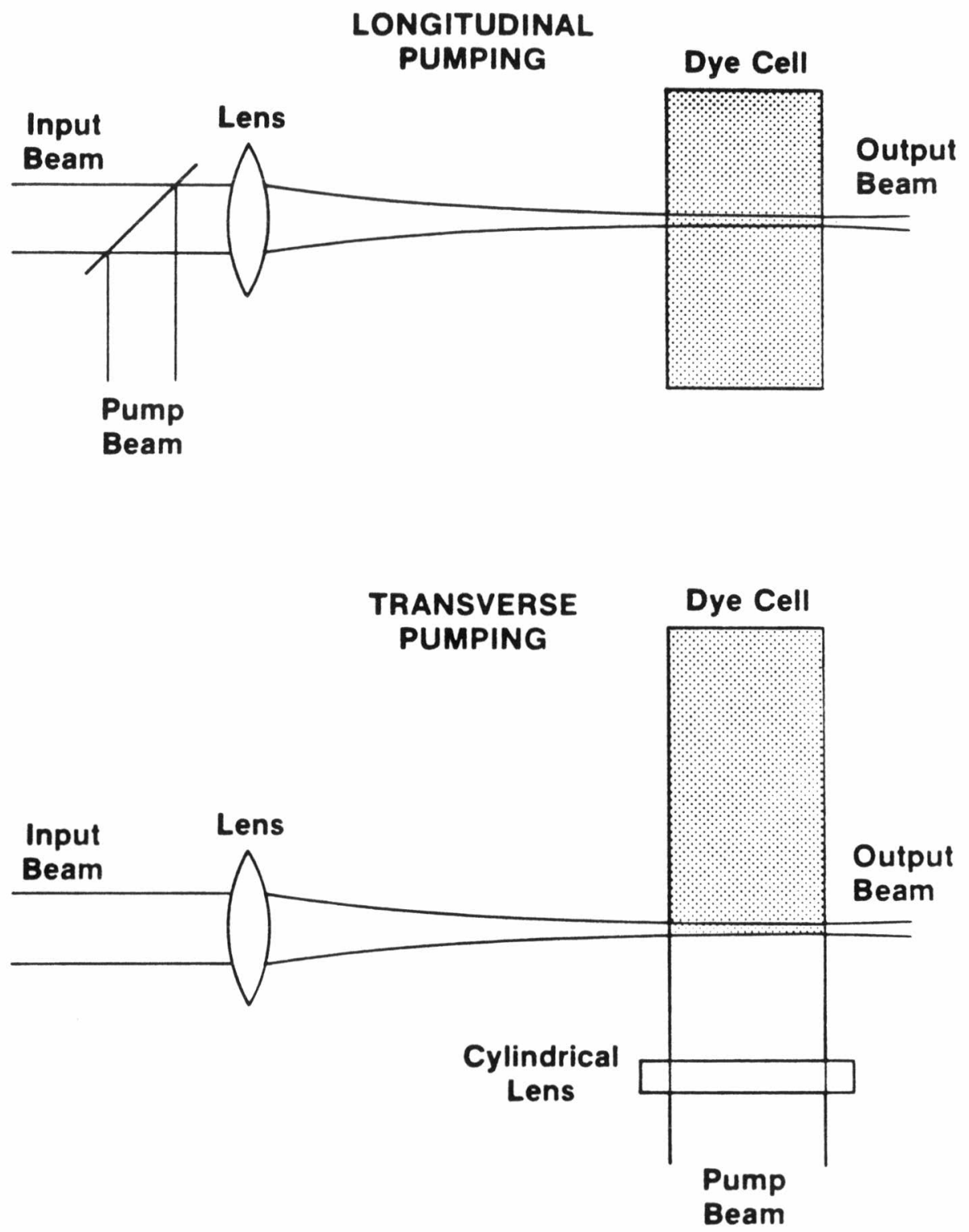

Figure 2-8 Comparison of pumping geometries 
changed without affecting the other.

Although most previous work has used transverse pumping, there are some major disadvantages. The greatest problem is with the pumping asymmetry. While the dye input spatial profile is a circular gaussian, the region of excited molecules is not. Since the cell is pumped from one side and the pump is absorbed as it propagates into the cell, the active region is shaped exponentially. The exponential shape does not provide an optimum match for the circular input beam and will cause asymmetries in the spatial profile of the amplified beam. A second more subtle disadvantage is that the absorption depth for the pump must be only a few tenths of a millimeter in the earlier stages, and more than an order of magnitude longer in the later stages. One solution is to use a short cell with a high concentration dye solution in the first stage and much longer cells with a lower concentration of dye in the later stages. The long cells greatly increase the path the subpicosecond pulse must travel through the dispersive solvent. An alternative solution persued by some workers (35) involves using different dyes in each stage. As a result, the absorption cross section can be decreased in the later stages without the requirements of low concentrations and long cells. The use of multiple dyes, however, is inconvenient. 
The advantages of longitudinal pumping are better beam quality and better timing between the pump and input pulses. Unlike transverse pumping, longitudinal pumping creates a circularly symmetric active region in the amplifier. The circular geometry provides the best match between the pump and input beam spatial profiles. The match provides more efficient amplification as well as preserving the circular spatial profile of the amplified beam.

Beam quality is an important consideration. For many experiments, high intensities are required and a good beam quality is needed to focus the amplified beam to a small spot. The beam quality of the amplified beam depends directly on the spatial quality of the pump beam in longitudinal pumping. In the third stage, where the pump and input beams are not focussed into the amplifier, the dependence on the pump comes about because each spatial portion of the amplified beam corresponds to a single spatial portion of the pump beam.

In the first two stages, where the pump and input beams are focussed into the amplifier, the amplifier acts as a spatial filter. When the pump beam is focussed, much of the energy is concentrated in a uniform central spot. Energy contained in higher spatial frequencies is directed into a larger area ${ }^{(63)}$ as shown in Figure 2-9. The energy in the larger spot will be less intense and not create much gain. 


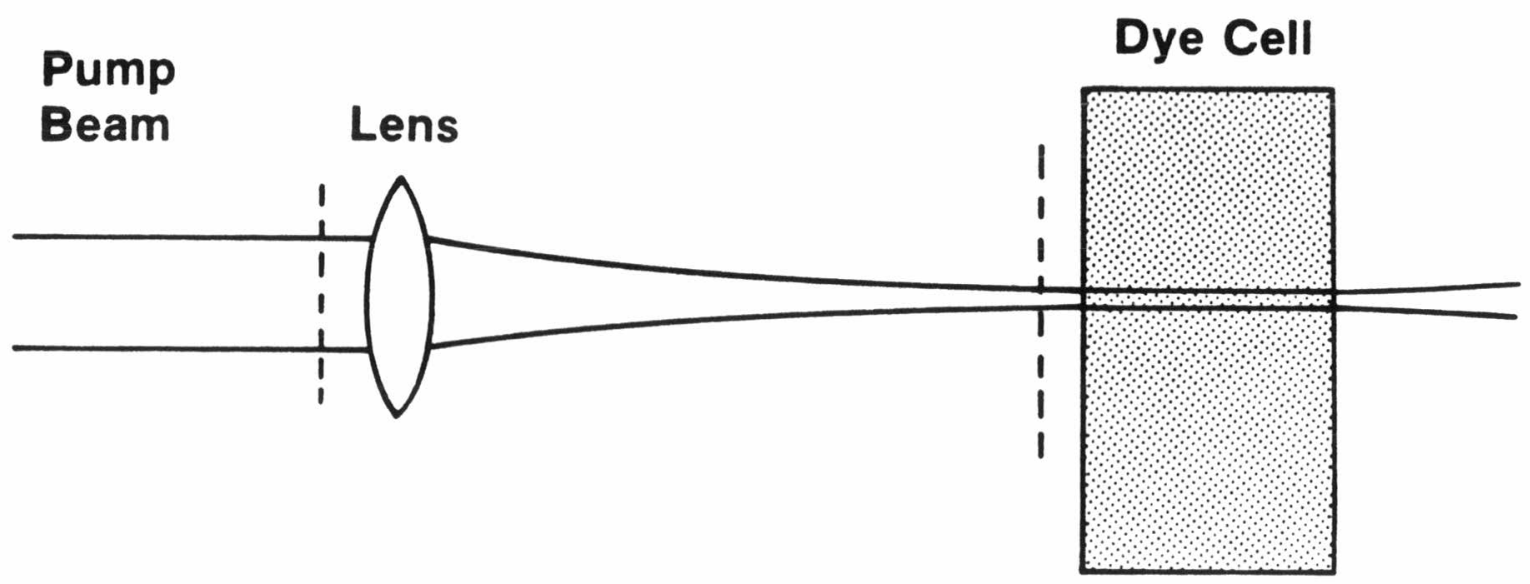

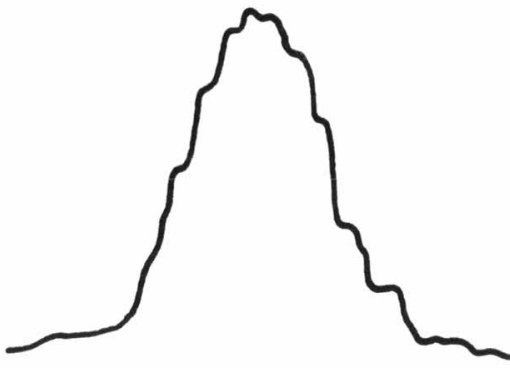

Pump Beam Spatial Profile

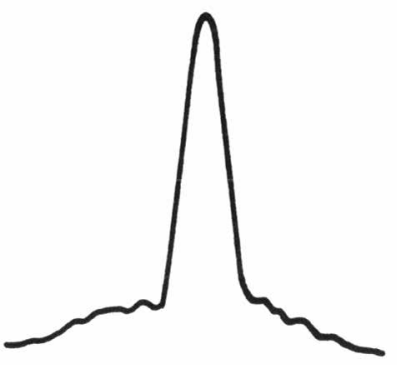

Focused

Pump Beam

Spatial Profile

Figure 2-9 Spatial profile of the pump beam 
The central area of high gain has an improved beam quality over the original pump beam; thus the focussing into the amplifier will filter out the high spatial frequencies in the pump beam. The input beam is focussed into the amplifier where it experiences a peak gain in the center with a gradually decreasing gain towards the edge of the active region. Because of gain saturation in the center of the beam, the gaussian pump beam will create a gain spatial profile that is approximately a super-gaussian. Thus, the input beam is focussed into an apodized aperture which will improve the amplified beam quality by spatially filtering the amplified beam in the amplifier itself. A super-gaussian apodized aperture is used to improve the beam quality in high power glass laser systems. (64)

With a high quality pump beam spatial profile, the amplified beam can easily propagate to the far field $(10 \mathrm{~m})$ without beam breakup. In the future, when a more energetic pump beam is obtained for our system, a spatial filter could be used to improve the pump beam spatial profile and hence the amplified beam spatial profile also.

Longitudinal pumping preserves the timing between the pump and input pulses by allowing them to propagate together through the amplifier. Instead of setting up gain throughout the amplifier at one instant, as with transverse pumping, longitudinal pumping creates the gain initially at 
the front of the cell and later at the rear of the cell. The input will always see the gain just after the pump pulse has passed. In addition, ASE launched from the rear of the cell will see less gain remaining; hence the ASE in the backward direction will be somewhat decreased.

One restriction of longitudinal pumping is the need for good dichroic mirrors to combine the pump pulse at $532 \mathrm{~nm}$ with the input pulse at $605 \mathrm{~nm}$. A special order filter was prepared by Tropel with the spectral characteristics shown in Figure 2-10. The filter was specified for use at 458 with S polarization and provides 968 transmission at $605 \mathrm{~nm}$ and 998 reflection at $532 \mathrm{~nm}$. These specifications included an antireflection coating on the rear surface.

\section{II.D.8 Pump Energy}

The pump beam must first be split into three portions to pump the three stages. The splitting is accomplished by passing the beam through partial reflectors which are antireflection coated on the rear surface. The quantity of pump energy must certainly increase in the later stages in order to pump an increased spot size. If we were to pump with equal intensity at each stage, given the respective spot sizes, we would split the energy 908, 98, and 0.18 for the third, second and first stages respectively. To obtain a 0.18 beam, however, is impossible in practice. In addition, we need to decrease the energy for the last stage 


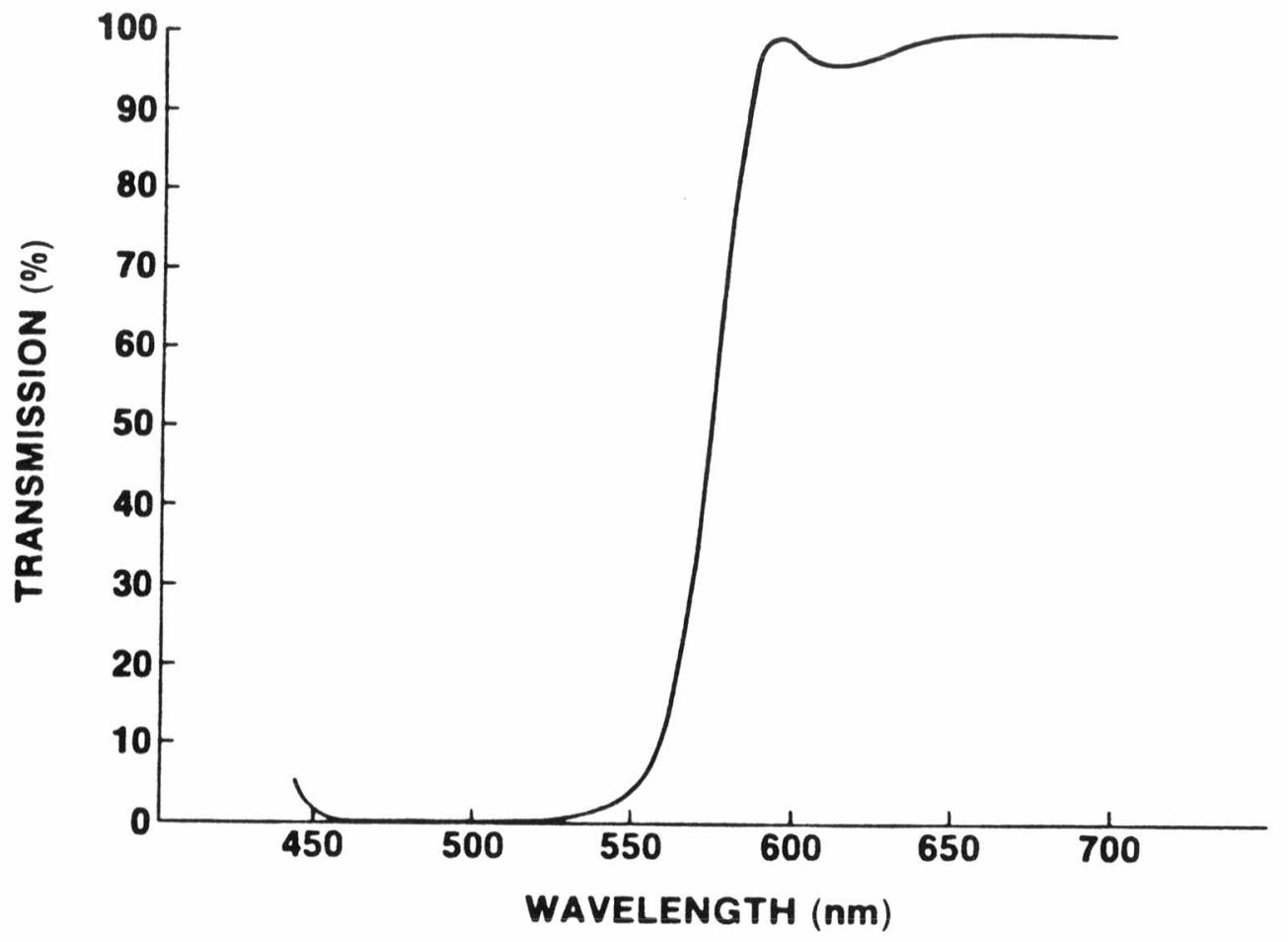

Figure 2-10 Spectral Characteristics of the dichroic mirror 
only slightly in order to pump much harder in the earlier stages. Borrowing somewhat from the last stage and based on the availability of partial reflectors, we have chosen to split the pump energy $70 \%, 20 \%$, and $10 \%$.

To achieve maximum overlap of the pump and input beams in the amplifier, the spatial mode of the two beams must be matched. The two spots are matched in size by varying the telescopes placed in each beam. The spot sizes can be measured using a moving edge and a detector.

\section{II.D.9 Alignment Procedure}

For each stage, the pump beam is overlapped with the input beam at the dichroic mirror. At the dichroic mirror, the two beams are aligned to be collinear using the following alignment proceedure. First, the dichroic mirror is placed at the angle which yields the greatest transmission for the input wavelength. The optimum angle is slightly less than 45 degrees. Next, the partial reflector which controls the angle of the pump is moved to a position so that 1) the beams overlap at the dichroic and 2) the beams leave the dichroic in approximately the same direction. A mirror is then inserted after the dichroic and the two beams are sent to a distant point $(10 \mathrm{~m})$. The angle of the partial reflector and the dichroic mirror are varied iteratively. The dichroic is used to overlap the beams at distant conjugates and then the partial reflector corrects 
the mismatch created at the dichroic. Only two or three adjustments to each mirror are required. The alignment proceedure can be used with either the high repetition rate beam from the RF coupled system or with the low repetition rate, high energy beam from the YAG amplifier. Typically, the high repetition rate beam is used to prealign the system and then the low repetition rate beam is used for final alignment.

Once the two beams have been made collinear, they are focussed together into the amplifier stage with a single positive lens. The position of the amplifier cell relative to the focus of the lens may be varied. This allows a change in spot size to occur without changing the alignment or the relative timing of the pump and input pulses. The focal length for the lens is chosen both because of the size of the focal spot and because of space limitations in setting up the amplifier chain. The focal length of the lenses before the first and second stages are $100 \mathrm{~mm}$ and $166 \mathrm{~mm}$ respectively. There is no lens used before the third stage.

II.D. 10 Timing

Now that the pump and input beams have been overlapped in space they must be overlapped in time. For the optically synchronized amplifier system the relative timing is accomplished with an optical delay. The delay consists of a 
roof prism on an optical rail and alignment mirrors to make the pump beam collinear to the rail. When the position of the prism is moved, the arrival time of the pump can be varied while the spatial alignment stays fixed. The time delay is continuous and can be measured by converting the centimeter scale on the rail to picoseconds $(1 \mathrm{~cm}=33.3 \mathrm{ps})$.

A different method was used to vary the relative timing of the pump and input pulses in the RF synchronized amplifier system. An electrical delay is placed in the RF line to one of the $\mathrm{CW}$ modelocked lasers. By varying the delay, the phase of the RF signal is changed continuously causing a relative delay in the pump beam. This arrangement is more convenient than the optical delay line and does not introduce the chance of optical misalignment.

Now that we have a means for varying the delay of the pump pulse, we may optimize the timing by measuring the amplification. First, a diode is used to observe the pump and input beams. The two beams can be temporally overlapped to approximately 500 ps using the diode and an oscilloscope. Next the gain in the amplifier is observed as a function of the relative delay and an optimum is found. Small adjustments are required from time to time as drift in the lasers occurs or the dye amplifier parameters are changed. Using the gain measurement, the timing can be fixed to better than $100 \mathrm{ps}$. 
Once the timing has been accomplished for the first stage, the second and third stage must be matched to the same timing. To match the second stage timing, the pump is blocked to the first stage and only the second stage is pumped. The timing is optimized in the same manner as the first stage and the optimum position of the delay line for the second stage is noted. The difference in the optimal timing position for the first two stages is calculated and the partial reflector that splits off the pump beam and directs it to the second stage is then moved to compensate for the timing difference. The second stage is then realigned following the proceedure outlined above and the timing is rechecked. If it does not yet match the first stage timing then the entire proceedure is repeated. Finally, the third stage is timed in the same manner. The overall timing proceedure is slow but once accomplished does not need to be repeated for many months.

\section{II.D.11 Concentration}

The free parameters may now be varied to optimize the amplifier. The dye concentrations are slowly increased in each stage and the gain is monitored. The results for the first stage are shown in Figure 2-11. At low concentrations, much of the pumping energy passes through the amplifier since there are not enough molecules present to absorb it. As the concentration increases, the number of 


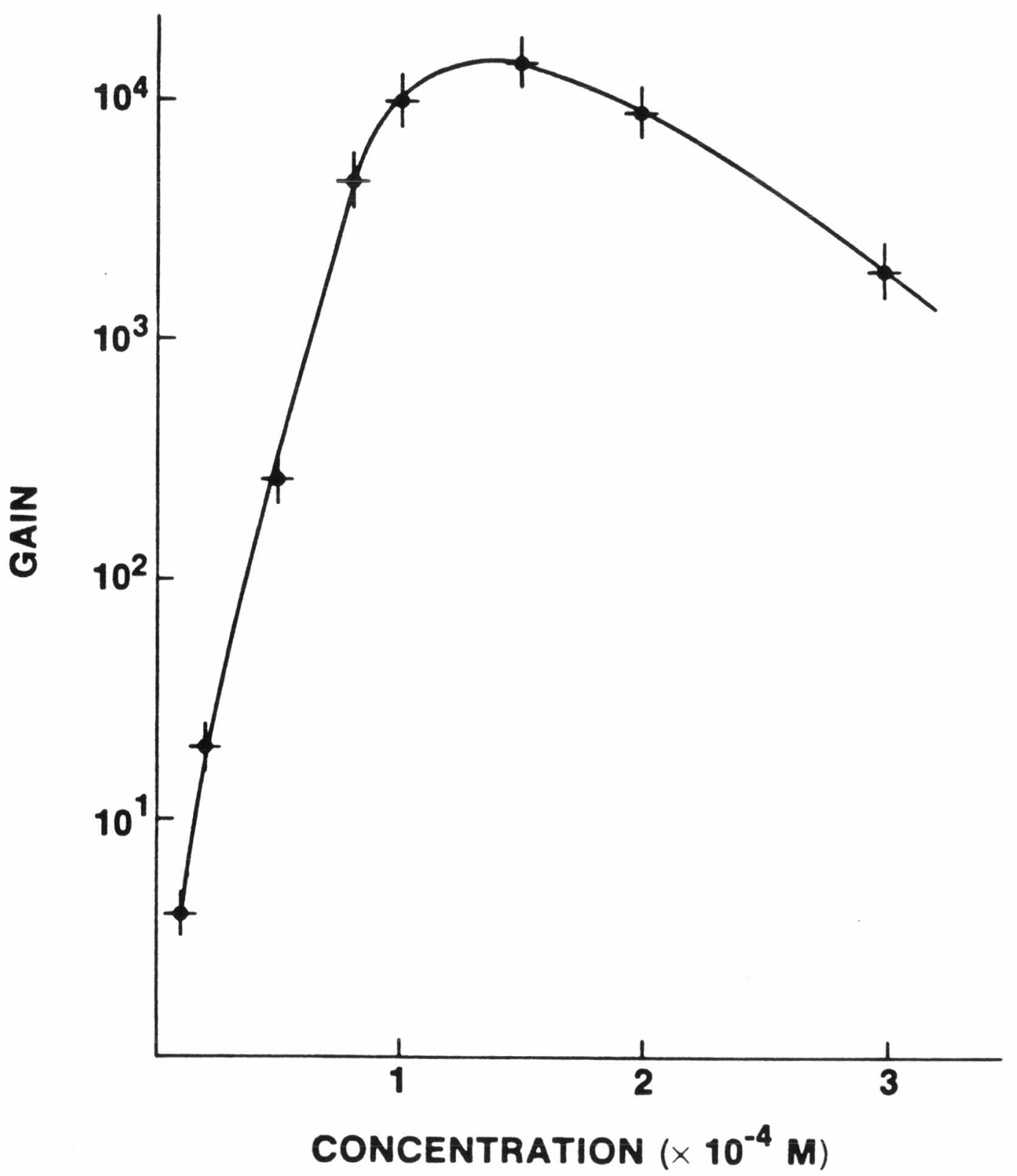

Figure 2-11 Peak gain as a function of the concentration 
excited molecules increases and, hence, so does the gain. Finally, the pump is completely absorbed and more molecules present will not mean any increase in the number of excited molecules. There is a reabsorption associated with these unpumped molecules, however, because the absorption and emission bands overlap for the dye. As a result, the gain decreases slightly at higher concentrations.

Using the method described above, the optimum dye concentrations were found to be $2 \times 10^{4} \mathrm{M}, 2 \times 10^{4} \mathrm{M}$ and $1 \times 10^{4} \mathrm{M}$ for the first, second and third stages. These values depend on the choice of cell length and the pump intensity that has been chosen for each stage.

II.D.12 Spot Size

The relay optics required to pass the input beam from one stage to the next are shown in Figure 2-12. The relative spot sizes for the pump and input pulses were adjusted with telescopes before the amplifier system. The actual spot sizes were then adjusted by moving the first two stages and maximizing the gain. The spot sizes at the lenses and at the third stage were measured with a variable pinhole to obtain the 908 energy point and then the full width half maximum (FWHM) was calculated. The spot sizes at the first two stages were measured with a sliding edge and a PIN diode. Their sizes are the full width half maximum also. 


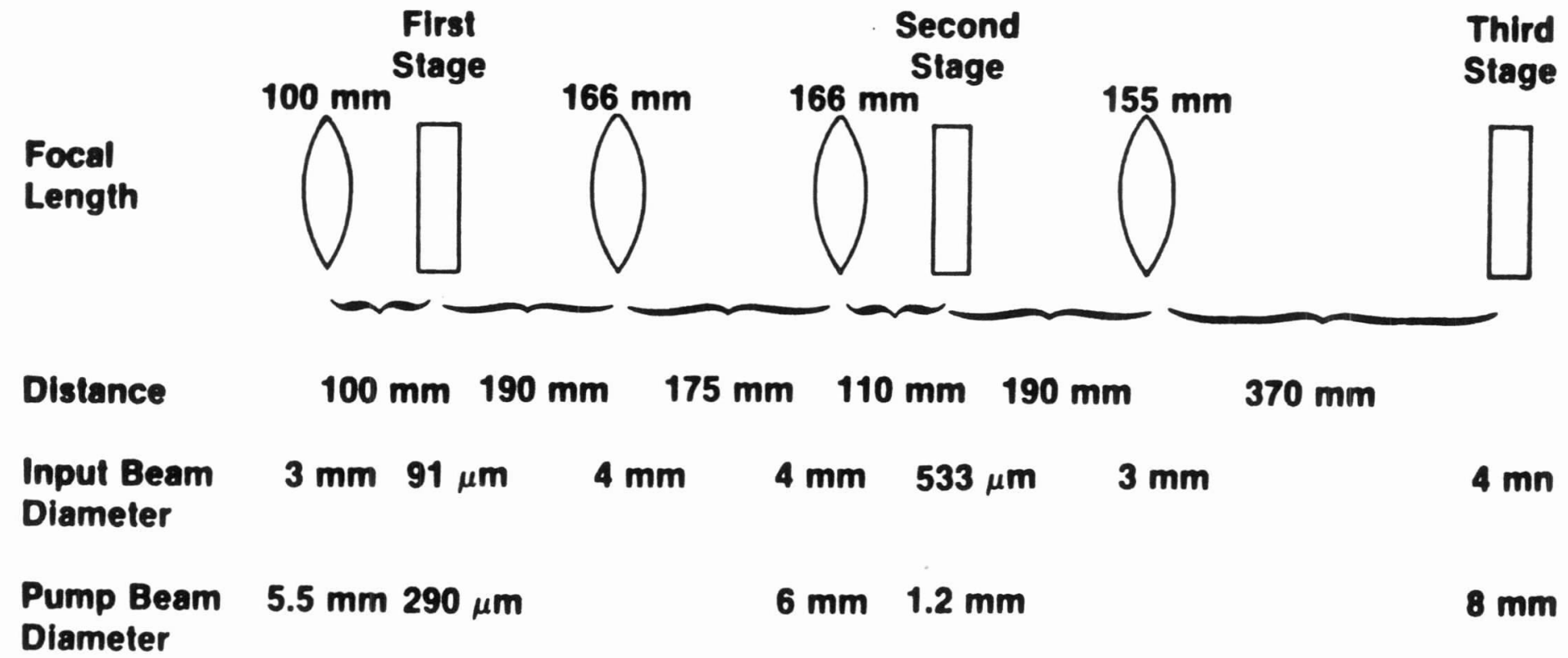

Figure 2-12 Dye amplifier relay optics 
The input beam is combined with the pump beam and then focussed into the first stage with a $100 \mathrm{~mm}$ focal length lens. The first face of the dye cell is placed near, but not at, the focus for an optimal spot size of $91 \mu \mathrm{m}$ for the input beam and $290 \mu \mathrm{m}$ for the pump beam. The amplified beam is then recollimated with a $166 \mathrm{~mm}$ lens. The pump beam for the second stage is then combined with the input beam and focussed with another $166 \mathrm{~mm}$ lens. The second stage is now operated well short of the focal region. The spot sizes are $533 \mu \mathrm{m}$ for the input beam and $1.22 \mathrm{~mm}$ for the pump beam. In order to work at the focal point of the lens and still get these spot sizes, too much space would be needed on the optical table. The output of the second stage is recollimated by a $155 \mathrm{~mm}$ lens and combined with the third stage pump beam at the final dichroic mirror. These beams are then fed directly into the third stage yielding a $4 \mathrm{~mm}$ spot size for the dye and an $8 \mathrm{~mm}$ spot size for the pump.

It is interesting to note that the ratio of the pump spot size to the input spot size is optimal at 2 to 1 and not 1 to 1 as might be expected. Other workers have also mentioned the need to pump a larger area. (35) The underlying reason is that the pump intensity and gain are not related linearly. Since the pump beam is spatially gaussian, the intensity is not high at the edge of the beam. As a result there is little gain at the edge of the active region compared with the center. The need to overfill the 
pump beam can also be seen experimentally. When the pump and input spot sizes are set 1 to 1 in the last stage, the amplified beam is much smaller in size than the input beam since only the center is amplified to any degree.

II.D.13 Gains

The gains are approxomately $10^{4}, 10^{2}$ and 10 respectively for the three stages. If the first stage is performing particularly well, the latter stages will be pushed further into saturation and will have a slightly lower gain. Thus, while the exact value of the gain for each stage may fluctuate slightly with alignment, a system gain of $10^{6}$ was easily attainable and reasonably stable. Typical energies, including all the losses, are measured and calculated to be as follows. The first stage input is $240 \mathrm{pJ}$. The second stage has $1.6 \mu \mathrm{J}$ input and the third had $48 \mu \mathrm{J}$ input. The system output is $260 \mu \mathrm{J}$ for a gain of $10^{6}$. This gain value includes all the system losses. A detailed description of the behavior of the three amplifiers under conditions of decreasing pump and input energy will be given in Chapter III. The results quoted above are achieved with $11 \mathrm{~mJ}$ of pump energy and are summarized in Table 2-2. The areas calculated include a factor of 1.67 because the cells are at Brewster's angle. When the pump is increased to $18 \mathrm{~mJ}$, the system output reaches a value of $2 \mathrm{~mJ}$ for an efficiency of 10\%, which is the highest efficiency reported 


\begin{tabular}{|c|c|c|c|}
\hline & FIR & SECOND STAGE & THIRD STAGE \\
\hline Input Energy & $240 \mathrm{pJ}$ & $1.65 \mu \mathrm{J}$ & $47.5 \mu \mathrm{J}$ \\
\hline Output Energy & $2.1 \mu \mathrm{J}$ & $37 \mu \mathbf{J}$ & $169 \mu \mathrm{J}$ \\
\hline Input Area & $1.1 \times 10^{-4} \mathrm{~cm}^{2}$ & $3.7 \times 10^{-3} \mathrm{~cm}^{2}$ & $1.3 \times 10^{-1} \mathrm{~cm}^{2}$ \\
\hline Input Intensity & $1.1 \times 10^{6} \mathrm{~W} / \mathrm{cm}^{2}$ & $2.2 \times 10^{8} \mathrm{~W} / \mathrm{cm}^{2}$ & $1.9 \times 10^{8} \mathrm{~W} / \mathrm{cm}^{2}$ \\
\hline Output Intensity & $9.7 \times 10^{9} \mathrm{~W} / \mathrm{cm}^{2}$ & $4.8 \times 10^{9} \mathrm{~W} / \mathrm{cm}^{2}$ & $6.8 \times 10^{8} \mathrm{~W} / \mathrm{cm}^{2}$ \\
\hline Pump E & $800 \mu \mathbf{J}$ & $1.6 \mathrm{~mJ}$ & $8.8 \mathrm{~mJ}$ \\
\hline Pump Area & $1.1 \times 10^{-3} \mathrm{~cm}^{2}$ & $1.9 \times 10^{-2} \mathrm{~cm}^{2}$ & $5 \times 10^{-1} \mathrm{~cm}^{2}$ \\
\hline Pump Intensity & $1.0 \times 10^{10} \mathrm{~W} / \mathrm{cm}^{2}$ & $1.2 \times 10^{9} \mathrm{~W} / \mathrm{cm}^{2}$ & $2.5 \times 10^{8} \mathrm{~W} / \mathrm{cm}^{2}$ \\
\hline
\end{tabular}

Input Pulse Width

Pump Pulse Width

$\begin{aligned} 2 \text { ps } & 1 \mathrm{~W} / \mathrm{cm}^{2}=10^{7} \mathrm{ergs} / \mathrm{sec} \mathrm{cm}^{2} \\ 70 \text { ps } & \end{aligned}$

Table 2-2

Pump and input parameters for the synchronous amplifier 
for a subpicosecond amplifier system.

II.D.14 Amplitude Fluctuations

Several factors contribute to the amplitude fluctuations of the output pulse. The major sources of fluctuations are the CW YAG oscillator and the regenerative amplifier. The amplitude fluctuations in the CW YAG oscillator are approximately plus or minus 18 and are due to the power supply. The time scale of these fluctuations are $60 \mathrm{~Hz}$ and $360 \mathrm{~Hz}$. There are also fluctuations in the kilohertz regime but these can be largely eliminated by adjusting the cavity length. The $1064 \mathrm{~nm}$ pulses are frequency doubled and the $532 \mathrm{~nm}$ beam amplitude fluctuations are plus or minus $2 \%$ due to the nonlinear effect of the frequency doubling. The green beam is used to pump the subpicosecond oscillator. Since the oscillator is operated near threshold, the fluctuations increase typically to plus or minus 58 .

The regenerative amplifier produces infrared pulses with plus or minus $5 \%$ fluctuations. The major source of fluctuations is the variation in the timing of the krytrons (see section II.C.2). If the timing window created by the Pockels cell varies, the output pulse from the regenerative amplifier will also. Frequency doubling the infrared pulse does not increase the fluctuations because we are operating on the linear portion of the conversion curve (Figure 2-4). 
The dye amplifiers are then operated using the pump and input pulses described above. Typical fluctuations of the amplified pulse are plus or minus 128. Averaging of ten shots is usually sufficient to provide accurate data (see Section II.D.16).

\section{II.D.15 Nonlinear Index Effects}

The nonlinear index induced by the optical intensity in the amplifier can cause whole beam and small scale self focussing. (65) self focussing causes a degradation in beam quality and places an upper limit on how intense the pump and input pulses can be. In designing an amplifier system with good beam quality, care must be taken to restrict the pump and input pulse intensities to a level where nonlinear index effects are minimal. High intensities in the amplifier create an increase in the index of refraction, which induces a phase distortion. The nonlinear phase distortion or "B integral" can be calculated using Equation 2-2. When $B=2 \pi$ a full wave of retardance is introduced.

$$
B=\frac{2 \pi}{\lambda} \int_{0}^{L} \gamma I(l) d \ell
$$

where $I(l)$ is the intensity in Watts $/ \mathrm{cm}^{2}, L$ is the length of the amplifier and

$$
\gamma=\frac{\left(4.19 \times 10^{-3}\right) n_{2}}{n_{d}}
$$


The linear index $\mathrm{n}_{\mathrm{d}}$ for water is 1.334 while the total nonlinear index $\mathrm{n}_{2}$ is $0.136 .(66)$

Using Equations 2-2 and 2-3 and the data from Table 2-2, I have calculated the B integral for the system of three dye amplifiers. To simplify the calculation I will assume that the intensity is constant throughout the amplifier, that is

$$
\int_{0}^{L} I(l) d l=I_{M} L \text {. }
$$

By letting $I_{m}$ be the maximum intensity in the amplifier, I will obtain an upper limit for the $B$ integral. Using the pump intensity at the front of each stage a value of $B=0.5$ is calculated for the system. When $B=6, a$ full wave of phase retardance is introduced. Thus the pump beam introduces distortions of less than $\lambda / 10$.

Referring again to Table 2-2 we may calculate the phase distortion due to the input pulse. I have used the output intensity for each stage to calculate an upper limit value of $B=0.6$. If, however, the pulsewidth becomes shorter, the B integral will increase proportionally.

$$
\text { B } \propto \frac{1}{\text { pulsewidth }} \text {. }
$$


For a $70 \mathrm{fs}$ input pulse, the $B$ integral would become 18 which implies 3 waves of retardance for the entire system. Much above this level, the phase distortion will dominate the beam quality and lead to beam breakup. To avoid beam distortion the input intensity would have to be decreased by going to larger spot sizes.

\section{II.D.16 Autocorrelation}

The job of the amplifier system is to amplify the input pulse while preserving the temporal profile and spatial profile of the pulse. The pulsewidth was measured both before and after amplification when the gain was $10^{6}$. The method used was the background free second harmonic generation (SHG) technique.(28)

Using this technique the following function is measured

$$
f(\tau)=G^{2}(\tau)+r(\tau)
$$

where $\mathrm{G}^{2}(\tau)$ is the second-order autocorrelation function of the pulse and $r(\tau)$ is a rapidly varying term that averages to zero. The delay $\tau$ is varied by changing the path length traversed by one of two identical subpicosecond pulses.

A diagram of the experimental setup is shown in Figure 2-13. The pulse to be measured is incident on the 508 beam splitter of a Michelson interferometer creating two identical pulses. The two subpicosecond pulses then 


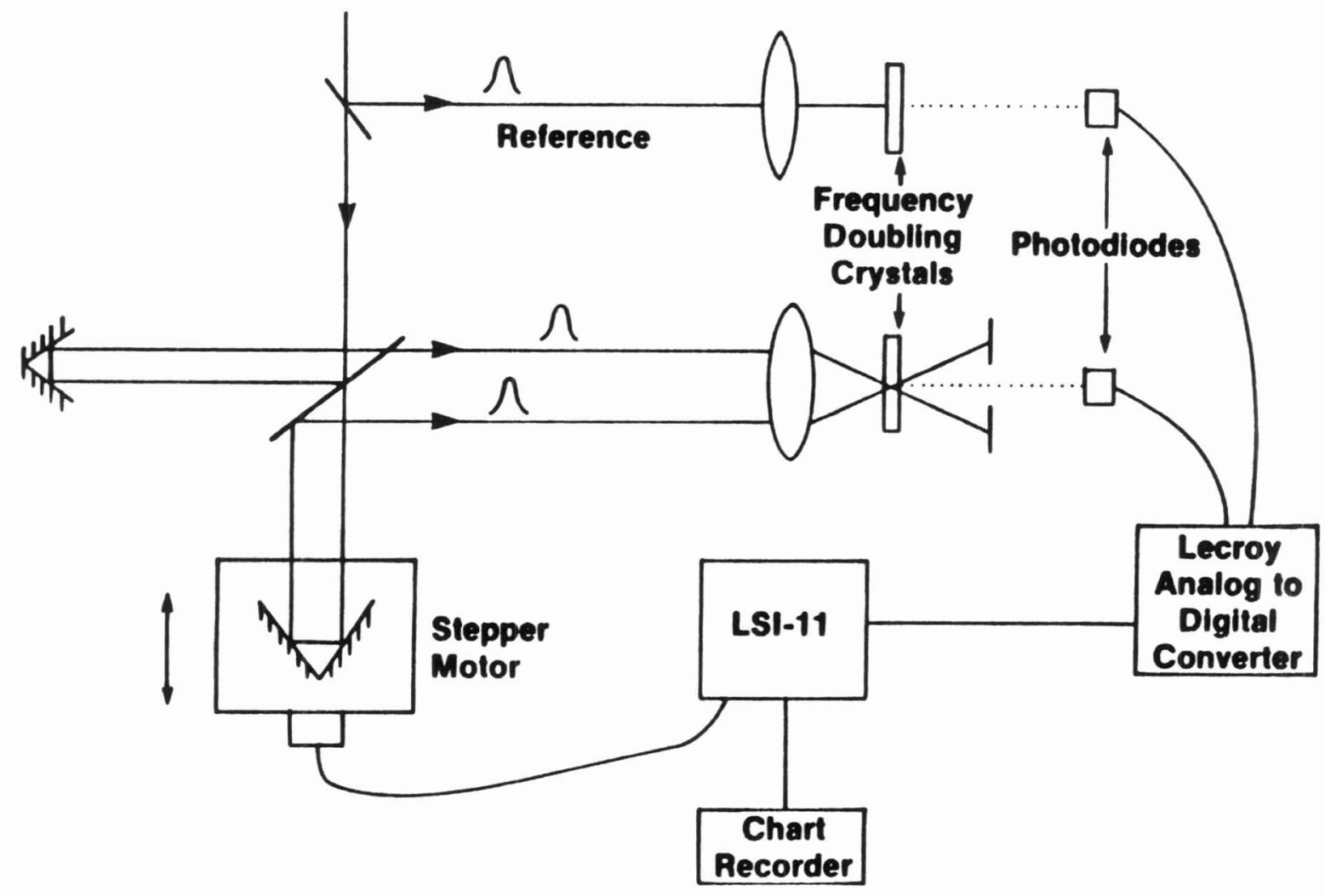

Figure 2-13 Experimental setup for the autocorrelation measurement 
traverse two different paths and return to the beam splitter after which they travel collinearly. A stepper motor is used to accurately control the delay between the pulses. In this manner, an accurate measurement of distance is used to measure time. The two ultrashort pulses are focussed into a thin frequency doubling crystal (KDP). Three frequency doubled beams are observed when the crystal is phase matched correctly. The careful alignment required is one drawback of the technique. The two outer beams are due to second harmonic generation from each of the input beams. The third, central spot is due to SHG from both beams, and the two beams must coincide in time for the third spot to be observed. It is the intensity of this signal as a function of the delay which gives the autocorrelation. A pinhole is used to separate the third beam from the others (hence background free) and the signal is interrogated by a photodiode.

Repetitive measurements are then made for different values of the delay $\tau$ until the entire correlation function is obtained. From the correlation function alone it is not possible to determine the exact pulse shape. (67) Instead, if a shape is assumed based on theory, the pulsewidth can be calculated. The difference between assumed shapes yields less than a factor of two difference in pulsewidth. For our measurements we have assumed a hyperbolic secant squared pulse shape $(33,36)$ which yields an actual pulsewidth 1.55 
times less than the direct autocorrelation pulsewidth measurement. (43)

The signal processing depends on the repetition rate of the pulse to be measured. To measure the pulsewidth before amplification, the oscillator is measured at $10^{8} \mathrm{~Hz}$. The continuous signal from the diode is sent to the $Y$ axis of an oscilloscope. A motor is used to vary the path length of one arm in the interferometer. The $x$ axis of the oscilloscope is driven by a transducer connected to the arm. A single trace of the autocorrelation can be accomplished in approximately 1 second. The autocorrelation of a $200 \mathrm{fs}$ input pulse is shown in Figure 2-14. The input pulse was then amplified by a factor of $10^{6}$ in the amplifier system.

The repetition rate for the amplified pulse is only $5 \mathrm{~Hz}$. To achieve a good autocorrelation, the deleterious effects of laser fluctuations and noise must be reduced. Special UV photovoltaic diodes (EG and G UV-100BG) were used as detectors. In addition a reference was set up to monitor any fluctuations in the amplified pulse. An identical focussing lens, doubling crystal and diode were used to duplicate the signal from the interferometer. When the crystal was aligned properly, the reference was accurate to 58 and was used to normalize the signal from the correlator.

The signals from the diodes were sent to a Lecroy 2249 W analog to digital converter and then stored by the LSI-II 


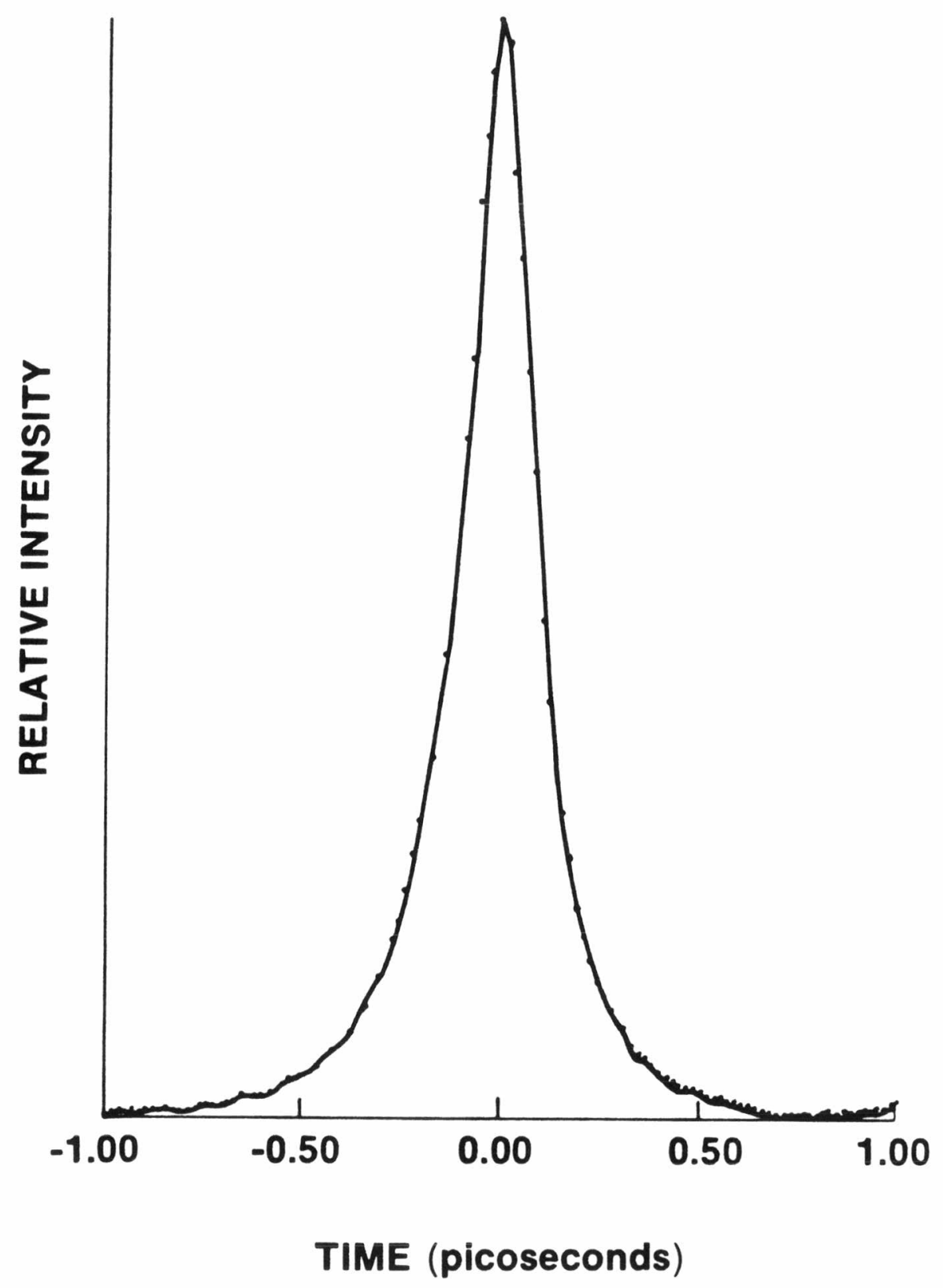

Figure 2-14 Oscillator pulse autocorrelation 
computer. Ten shots were averaged for a given delay and then the stepper motor was moved under computer control. At the new delay, ten more shots were averaged. This proceedure continued for approximately 15 minutes until a complete correlation curve was recorded.

In Figure 2-15 is the autocorrelation of the amplified pulse. If the same pulse shape is assumed, the pulsewidth is also $200 \mathrm{fs}$. The measurement was repeated several times with the result always being less than $300 \mathrm{fs}$. We conclude that with a gain of $10^{6}$, the temporal pulse shape of the subpicosecond pulse is preserved.

II.D.17 Amplified Spontaneous Emission (ASE)

An important consideration for any amplifier system is the quantity of amplified spontaneous emission generated. As was stated earlier, we anticipated a greatly reduced quantity of ASE because of the use of 70 ps pump pulses.

Significantly, no isolation is required between the amplifier stages. Systems that use nanosecond pump pulses are unable to function without isolation because the ASE will swamp the output pulse. (33) The output of the synchronous amplifier system typically has a ratio of output pulse energy to ASE energy of 4 to 1 . Of course the output pulse contains its energy in a few hundred femtoseconds while the ASE is contained in a 100 ps pulse. In addition, 


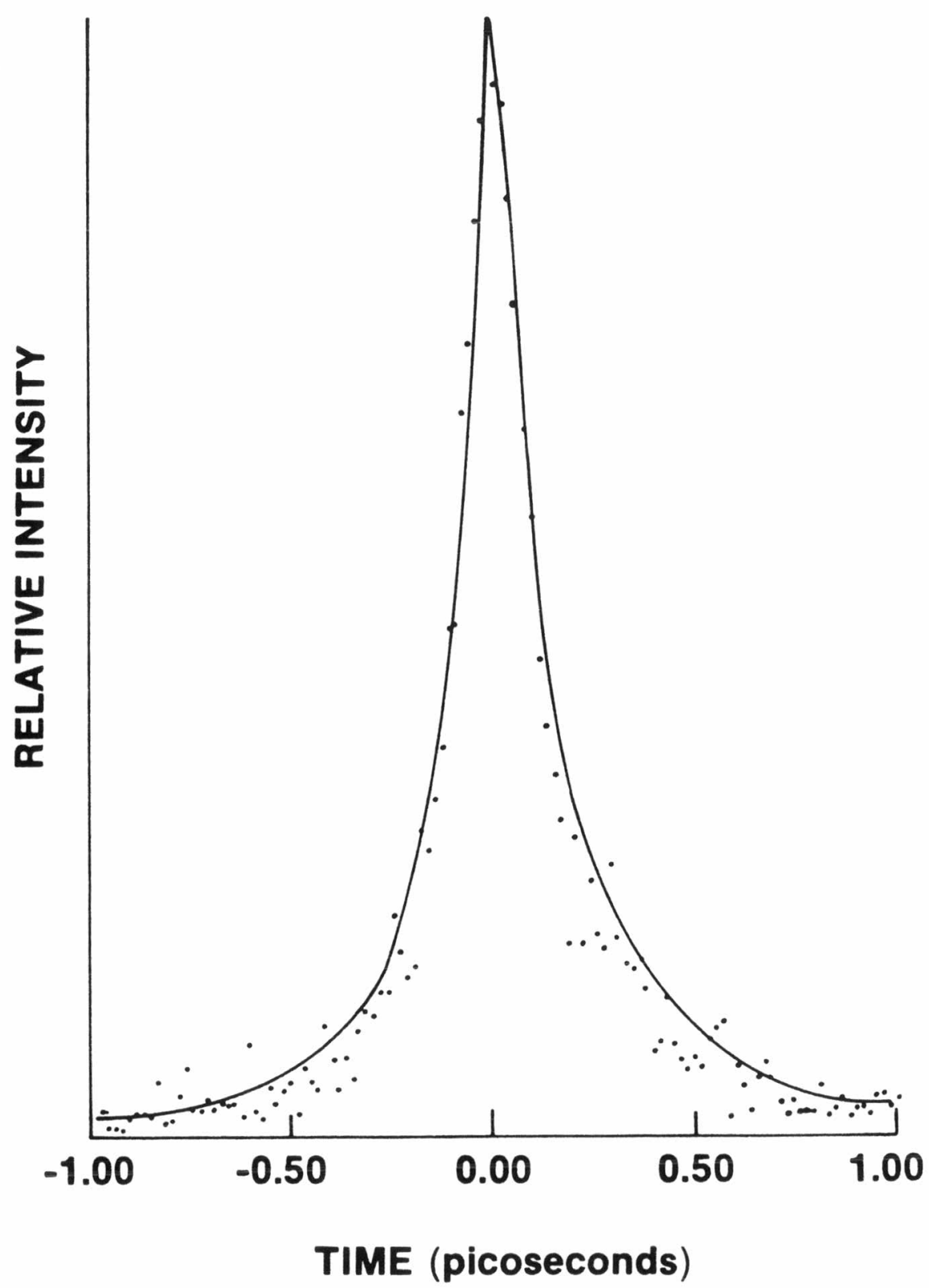

Figure 2-15 Amplified pulse autocorrelation 
the bulk of the ASE follows the output pulse. This fact was confirmed experimentally by observing the amplified pulse and the ASE with a streak camera. The results, where the amplified pulse was attenuated spectrally, are displayed in Figure 2-16. The pulse width of the amplified pulse is limited by the resolution of the streak camera while the pulse width of the ASE is temporally resolved.

For many experiments, a contrast ratio of 4 to 1 is not sufficient. The contrast ratio can be improved by using a saturable filter. Recall that the second stage was placed before the focal point of the beam. A 1 or $2 \mathrm{~mm}$ schott glass filter RG645 can then be placed at the focus and used as a convenient saturable filter. When the filter is inserted, the energy contrast at the end of the system can be increased to 1000 to 1. Typical results are shown in Table 2-3. The measurement of the ASE is obtained by blocking the dye input pulse to the amplifier chain. This measurement represents an upper value on the quantity of ASE since, with the input pulse present, the ASE will see a lower gain.

There are two factors which contribute to the reduced value of ASE in our system: the use of short pump pulses and the use of dichroic mirrors. Since the amplifiers are excited with shorter pump pulses, the population of the dye is inverted only once. With longer pump pulses (a few 


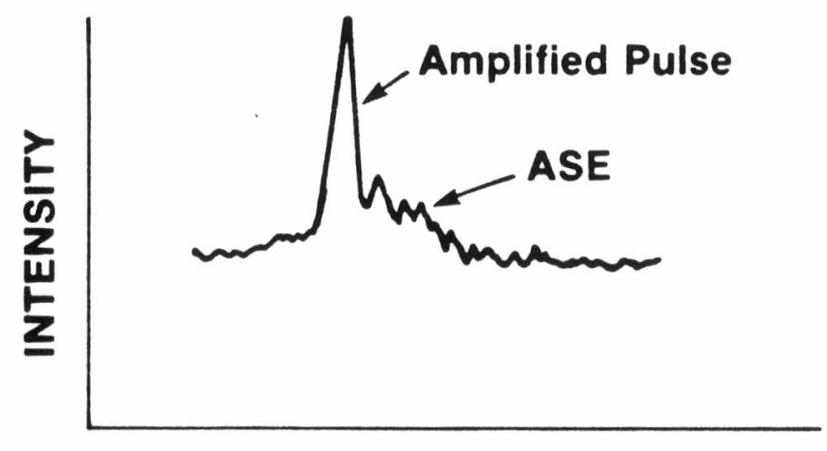

TIME

Figure 2-16 Streak camera measurement of the amplified pulse and ASE 


\begin{tabular}{lccc} 
& $\begin{array}{c}\text { AMPLIFIED } \\
\text { PULSE }\end{array}$ & ASE & CONTRAST \\
\hline \hline $\begin{array}{l}\text { Without } \\
\text { Filter }\end{array}$ & $1.55 \mathrm{~mJ}$ & $0.300 \mathrm{~mJ}$ & 5 \\
$\begin{array}{l}\text { With } \\
\text { Filter }\end{array}$ & $1.35 \mathrm{~mJ}$ & $0.006 \mathrm{~mJ}$ & 225
\end{tabular}

Table 2-3 Amplified spontaneous emission contrast ratio 
nanoseconds), the population can be recycled many times. Each time the molecules relax, they contribute to the ASE; thus, with $70 \mathrm{ps}$ pump, the quantity of ASE is greatly decreased.

One other method which can be used to discriminate against ASE, is spectral filtering, such as with the dichroic mirrors. Since the ASE is yellower than the amplified pulse, it sees a lower transmission through a spectral filter set to pass $605 \mathrm{~nm}$. The yellow shift is due to the choice of kiton red as the active medium. The dichroic filters discriminate against the ASE because of their spectral characteristics (Figure 2-8). A grating can also be used to separate the output pulse from the ASE but care must be taken not to limit the bandwidth of the subpicosecond pulse.

\section{II.E Summary}

In this chapter, I began with a description of the other systems that have been developed for the amplification of subpicosecond optical pulses to the gigawatt power level. The remainder of the chapter detailed our system and the choice of parameters that went into it. The system is broken into three subsystems: The oscillator, YAG amplifiers and dye amplifiers. By using synchronized 70 ps pump pulses, a high efficiency subpicosecond amplifier system was designed and investigated. The synchronous 
amplifier system constructed obtains an efficiency of $10 \%$, which is higher than any other subpicosecond gigawatt amplifier. In addition, we have constructed the only subpicosecond gigawatt amplifier system which can be operated without isolation between the amplifier stages. The amplifier systems features longitudinal pumping for better beam quality and picosecond synchronization for improved amplitude stability. 
CHAPTER III

DYE AMPLIFIER EXPERIMENTS

\section{III.A Introduction}

An efficient amplifier system can be built using short synchronized pump pulses. In Chapter III, I will present a series of experiments aimed at proving this thesis and understanding the basic physics of dye amplifiers. The most important quantities in assessing efficiency is the gain of an amplifier and the mechanisms that limit it. I have investigated several loss mechanisms and the speed at which the gain decreases. I have also investigated the limit that saturation places on the gain. These experiments support my supposition that synchronous amplification is more efficient than previous methods.

In the first part of this chapter, I investigate a single amplifier in detail. The results obtained here are applicable to any stage of the amplifier system. The results focus on the time dependent nature of the gain. In the second part, I present results that demonstrate the differences between the three amplifier stages with an emphasis on saturation. 


\section{III.A.I Input Parameters}

The parameters of interest are those that we discussed in Chapter I. The primary variable is the delay time between the pump and input pulses. It is by changing the delay time that we may obtain the time resolution needed to examine the physics. Other parameters that are varied include the pump and input pulse intensities and the solvent viscosity. In particular, the pump intensity can be used to vary the gain and the viscosity used to vary the molecular reorientation time.

\section{III.A.2 Output Parameters}

Since we wish to study the optimization and efficiency of the amplifiers, we measure the gain. The key distinguishing feature of the present work is the use of short pump pulses. Because the pump changes rapidly, the gain never reaches a steady state condition, as will be shown in section III.B.3. We may measure the time dependence of the gain or the peak value of the gain. In the first part of the chapter, the time dependence of the gain is preferable for displaying those characteristics common to all synchronously pumped amplifiers. In the latter part of the chapter, which concerns the difference between stages, I investigate the peak gain. 


\section{III.B Timing Curves}

Measurements of the transient gain can reveal the loss mechanisms that limit amplifier gain and efficiency. The transient gain, or timing curve, can be measured in a pump-probe experiment. A pump pulse followed by an input pulse is required for amplification. We may also consider the pump pulse as an excitation of a sample and the input pulse as a probe. In this view, the pump is perturbing a dye solution by exciting ground state molecules $s_{0}$ to the first excited state $S_{1}$. The probe pulse then comes at a later time and tests the population inversion. Depending on how much later the probe arrives, some portion of the excited dye will have relaxed back to the ground state. The amplification process thus can be viewed as a pump-probe experiment where we measure the transient gain.

\section{III.B.I Pump-Probe Experiment}

To perform a pump-probe experiment and determine the effective storage time of the amplifier, a few criteria must be met. The pump pulse must be of the appropriate wavelength to excite the dye from $S_{0}$ to $S_{1}$, the probe pulse must have the appropriate wavelength to interrogate the lasing transition and the two beams must overlap spatially in the sample. These requirements are also necessary for an efficient amplifier. The independent variable is the delay time between the pump and probe which is known to an 
accuracy of a few picoseconds.

\section{III.B.2 Apparatus}

The transient gain was measured using the layout shown in Figure 3-1. The pump pulse was prepared by the optically synchronized amplifier described in section II.C.l. The $532 \mathrm{~nm}$ pump pulse was linearly polarized and its intensity was adjusted with the use of calibrated neutral density filters. Because these filters contained a slight wedge, the system alignment was checked after any change in the filter stack. The probe pulse was prepared at $605 \mathrm{~nm}$ by the dye oscillator described in section II.B. The probe pulsewidth was between 1 and 2 ps for the experiments that will be described here. The probe pulse was sufficiently attenuated so that it would not saturate the amplifier.

The optical system shown in Figure $3-1$ was placed in the dye beam to provide a temporal delay without misalignment. An Aerotech stepper motor and platform with $2 \mu \mathrm{m}$ steps was used to increase the path length. The stepper was controlled by an LSI-ll computer and provided a delay of $200 \mathrm{ps}$. Multiple passes through the stage using cube corner reflectors were used to obtain a total delay of 1.6 ns with a resolution of $53 \mathrm{fs}$.

The probe beam was passed through a cube polarizer and a single order half wave plate to rotate the beam's 


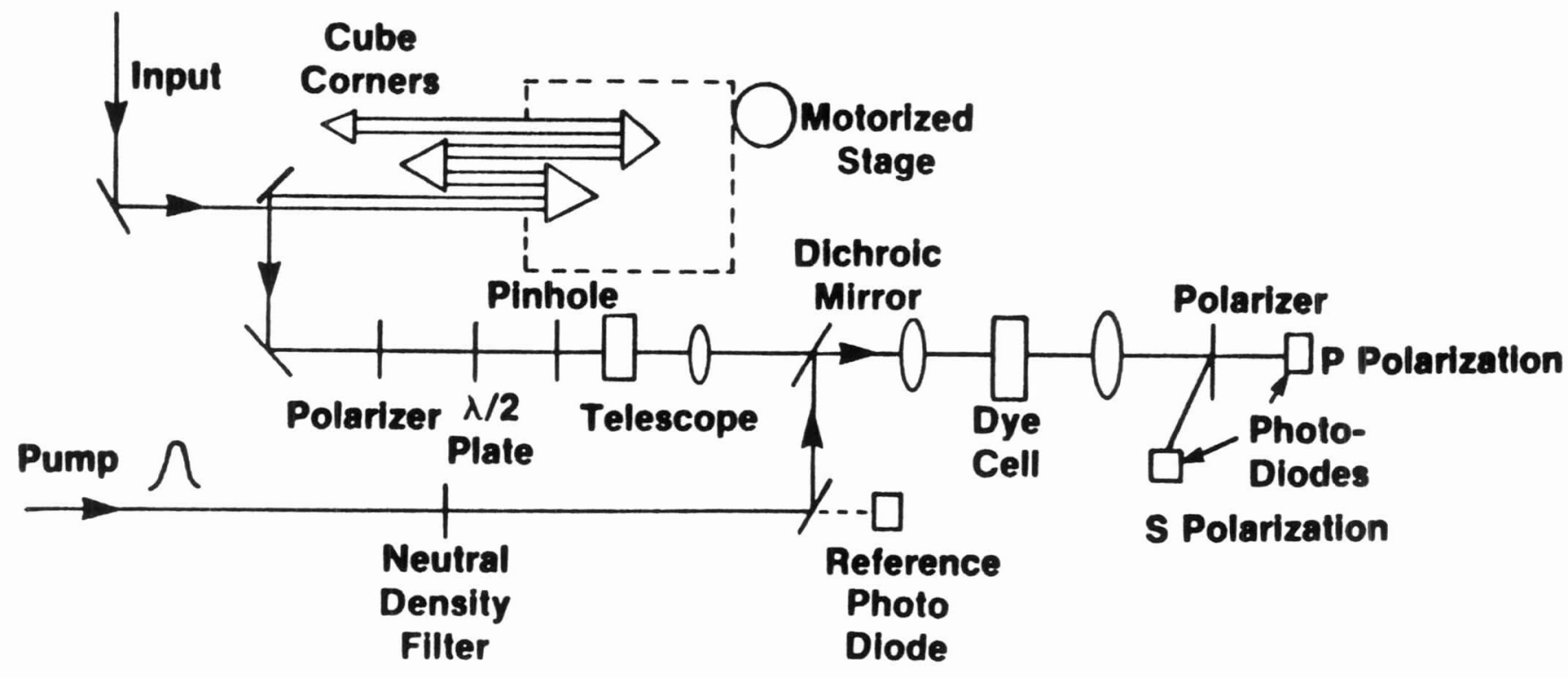

Figure 3-1 Experimental setup for measuring the transient gain 
polarization. Next, a telescope was used to expand the beam and mode match it to the pump beam at a dichroic mirror. The two beams were then focussed into a single dye cell using a series of different lenses so that the spot size could be varied. The dye cell was the standard $1 \mathrm{~cm}$ long flowing cell used in the large amplifier system. The output beam was collimated by a lens and passed through a cube polarizer. Either output polarization could then be observed by a PIN diode.

The experiment was controlled by the LSI-1I and a program witten in Forth. (68) The signal from the diode was sent to a gated Lecroy $2249 \mathrm{~W}$ analog to digital converter and stored by the computer. Generally 10 shots were averaged to obtain better signal to noise. A reference diode was used to sample the pump intensity and all shots below a preset level were disregarded by the program. After data had been collected for a given time delay between the pump and probe pulses, the computer would move the stepper motor to the next position and the data collection would begin anew.

\section{III.B. 3 Results}

A typical result for the transient gain in a high gain amplifier is shown in Figure 3-2. The individual data points are separated by 10 ps and the curve is drawn by hand. The zero timing mark corresponds to the beginning of 


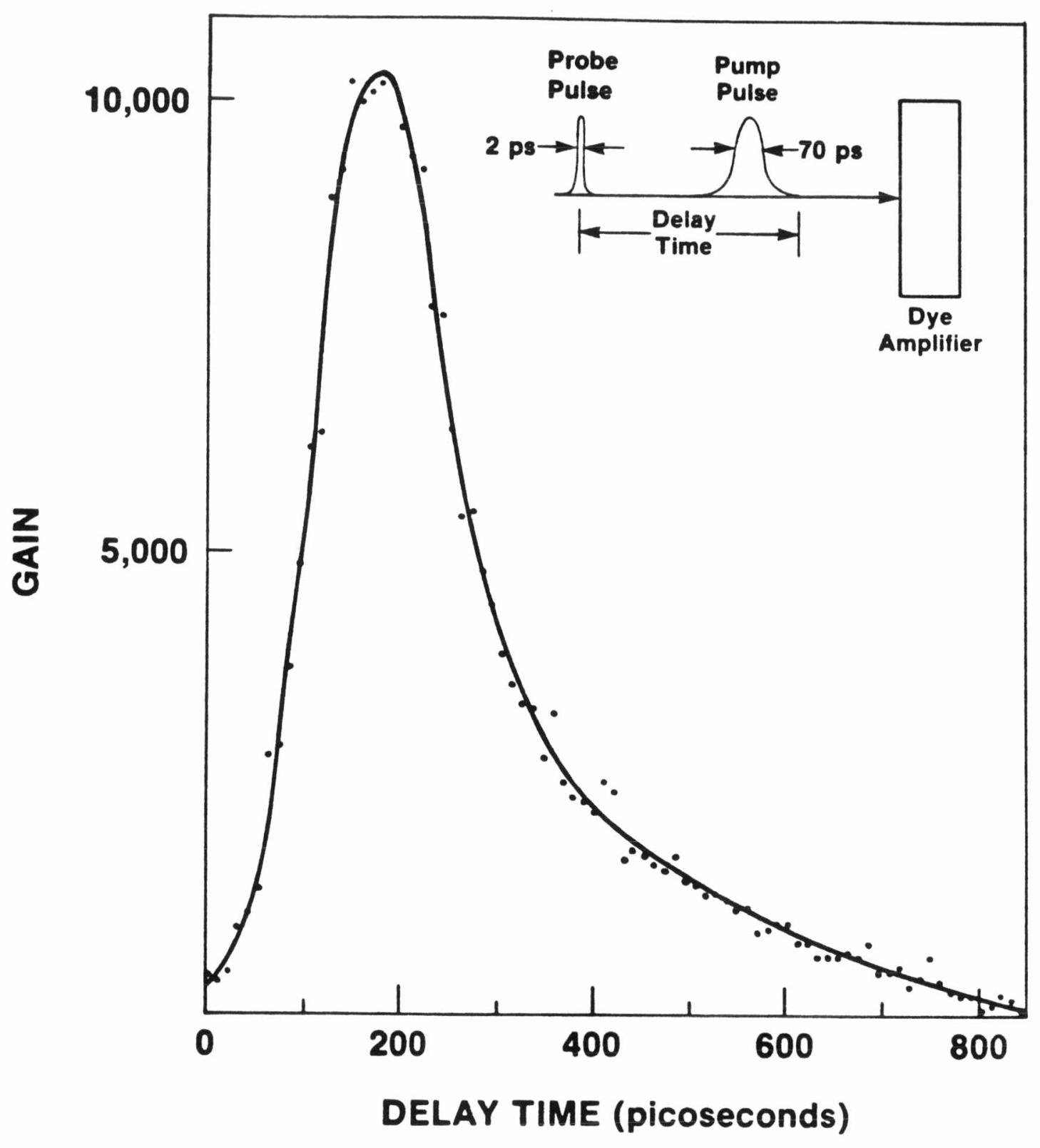

Figure 3-2 Transient gain in a dye amplifier 
the pump pulse. The main features of the transient gain can now be seen. The increase in gain occurs as the energy from the pump pulse is stored by the dye. The loss mechanisms (which include ASE) soon dominate and the gain decreases in a roughly exponential manner. The time scale for the loss is a few hundred picoseconds. The experimental data verifies the well known fact that dyes are not good energy storage media which is the reason that led us to pump with short synchronized pump pulses in the first place. clearly, if we were to use nanosecond pump pulses with a medium that stores energy for only a few hundred picoseconds, we would have a less efficient picosecond pulse amplification system. A theoretical efficiency comparison between $70 \mathrm{ps}$ and $7 \mathrm{~ns}$ pump pulses is given in section IV.F.

III.B.4 Gain Onset

Let us first investigate the onset of the gain. Assuming a gaussian pump pulse, no saturation and no losses during the pump pulse, we may write Equation 3-1 for the shape of the gain during the pump.

$$
\left.G(t)=e^{\left[\frac{A}{T \sqrt{ } \pi} \int_{-\infty}^{t} e^{-\left(\frac{x}{T}\right)^{2}}\right.} d x\right]
$$

where

$$
T=\frac{F W H M}{2 \sqrt{ } \ln 2}
$$


for the gaussian pump pulse and $A$ is a constant that depends on the peak gain. When the pump is finished at $t=\infty$ we find $G(t)=e^{A}=10,000$ from Figure $3-2$, and thus $A=9.21$. The full width half maximum (FWHM) of the gaussian pump pulse corresponds to the 108 to 908 rise of the integral of the pump which in turn corresponds to the $e^{\cdot 1 A}$ and $e^{.9 A}$ points in the gain. Using the value $A=9.21$ we find that the FWHM of the pump is the $0 \%$ to $40 \%$ rise points for the gain. From Figure 3-2, we obtain a FWHM of $89 \mathrm{ps}$ which is consistent with the experimental measurement of the pump pulse width. Autocorrelation measurements have shown the pump pulse to be between $70 \mathrm{ps}$ and $100 \mathrm{ps}$. We conclude that the rise of the gain is due to integrating the pump pulse energy and occurs largely before the losses have begun.

\section{III.C Loss Mechanisms}

of greater interest is the decrease in the gain. It is the shape and rate of the fall that gives insight into the loss mechanisms in the amplifier. The loss mechanisms I will investigate, fluorescence, molecular reorientation and ASE, are the limiting factors in the amplifier efficiency. Unfortunately, the loss mechanisms are not separable, that is, they all compete with each other. In the high gain regime, a certain loss will dominate and it is only by varying the input parameters that we can separate the effects of the different loss mechanisms. First I will 
discuss the three major loss mechanisms and how they arise. I will start with the slowest one and proceed towards the most rapid. Then I will display the results of a series of experiments in which the transient gain is measured but one of the input parameters is varied. By changing the pump intensity, solvent and pumping geometry, I will be able to demonstrate when each loss mechanism is dominant and measure the rate of that loss.

III.C.I Fluorescence

In some sense, the slowest loss mechanism is fluorescence. The molecules that have been excited to the first singlet state $S_{1}$ relax to the ground state $S_{0}$. The number of molecules per cubic centimeter (molecular density) that are excited, decays exponentially with a time constant of $2.8 \mathrm{~ns}$ for the dye kiton red. (69)

$$
N(t)=N_{0} e^{-\left(\frac{t}{T}\right)}
$$

Fluorescence decay means that less than half the excited molecules will be left only 2 ns after the pump pulse. Fluorescence establishes the upper limit for the storage time of the dye.

As the gain increases, however, the effect becomes more severe. Recall that the gain depends exponentially on the number of excited molecules as described in Chapter $I$. 


$$
\mathbf{G}=\mathbf{e}^{\mathrm{ON} \ell},
$$

where $\sigma$ is the emission cross section and $l$ is the amplifier length. Using Equation 3-3 we find that the gain depends on the exponential of the exponential of the decay time tau.

$$
G(t)=e^{\left[\sigma l N_{0} e^{-\left(\frac{t}{T}\right)}\right]}
$$

Although the fluorescence decays in $2.8 \mathrm{~ns}$, when $\sigma \mathrm{N} \ell$ is quite large the gain will decrease much more quickly. Calculations using Equation 3-5 are shown in Figure 3-3. As the pump increases so does the quantity $\mathrm{N}_{0^{\circ}}$. The time for $G(t)$ to reach $1 / \mathrm{e}$ of its initial value is then plotted and can be seen to decrease significantly below the $2.8 \mathrm{~ns}$ level. In a high gain amplifier, the losses due just to fluorescence can be quite severe.

\section{III.C. 2 Molecular Reorientation}

In a low gain amplifier, molecular reorientation can cause a faster loss than fluorescence. The dye amplifier is excited by a linearly polarized pump pulse. The cross section for molecular excitation decreases as the angle between the long thin molecule and the pump polarization increases. (70) An anisotropic distribution of excited molecules is created with a greater number being aligned along the direction of polarization of the pump beam. (7I) 


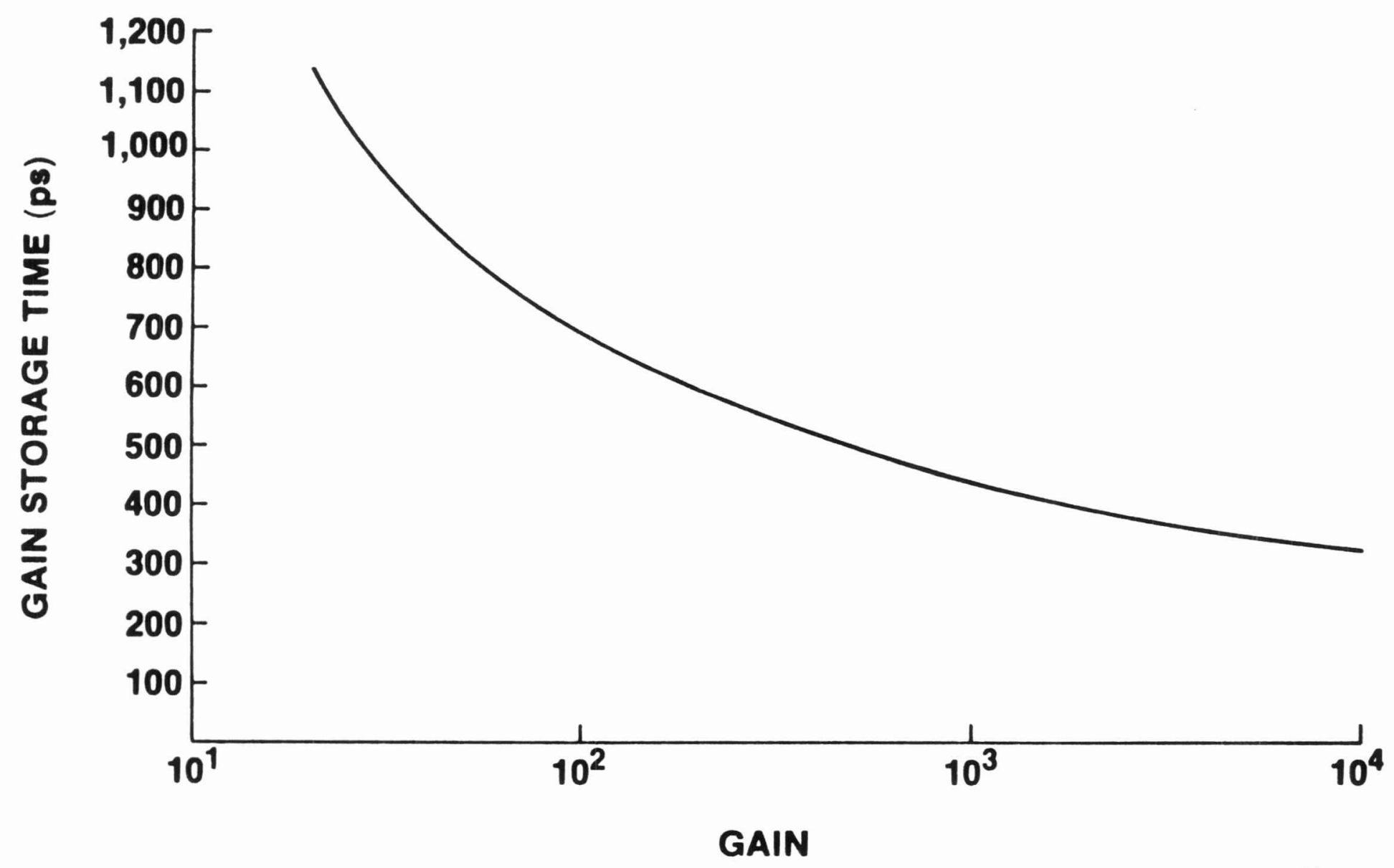

Figure 3-3 Fluorescence dependence of the gain storage time 
In time, however, these molecules rotate and randomize their orientation causing a loss of gain to the linearly polarized probe pulse. The speed of the reorientation is largely dependent on the viscosity of the solvent chosen. The experiments which will be presented in section III.D.l were done with water and ethylene glycol and in the low gain regime a difference between the reorientation times was observed.

An additional experiment was performed in a low gain amplifier to demonstrate that molecular reorientation was in fact being observed. Using the setup shown in Figure 3-1, the linear polarization of the probe beam was set at 45 degrees to that of the pump beam using a half wave plate. After the dye cell, a polarizer was used to split the input polarization into two beams of equal intensity: one polarized parallel to the pump, the other perpendicular. The peak gain for the polarization parallel to the pump $(G \|)$ was 240 and the peak gain perpendicular $\left(G_{\perp}\right.$ ) was 40 . The complete pump and probe experiment was then performed and the results are shown in Figure 3-4. When the data is normalized it is observed that the $G_{\perp}$ curve rises nearly as fast as the $G_{\|}$curve but decays more slowly.

Ideally the linearly polarized pump will generate a cosine $^{2}$ distribution of excited molecules. (70) If there is saturation, the distribution will be altered. (72) when the 


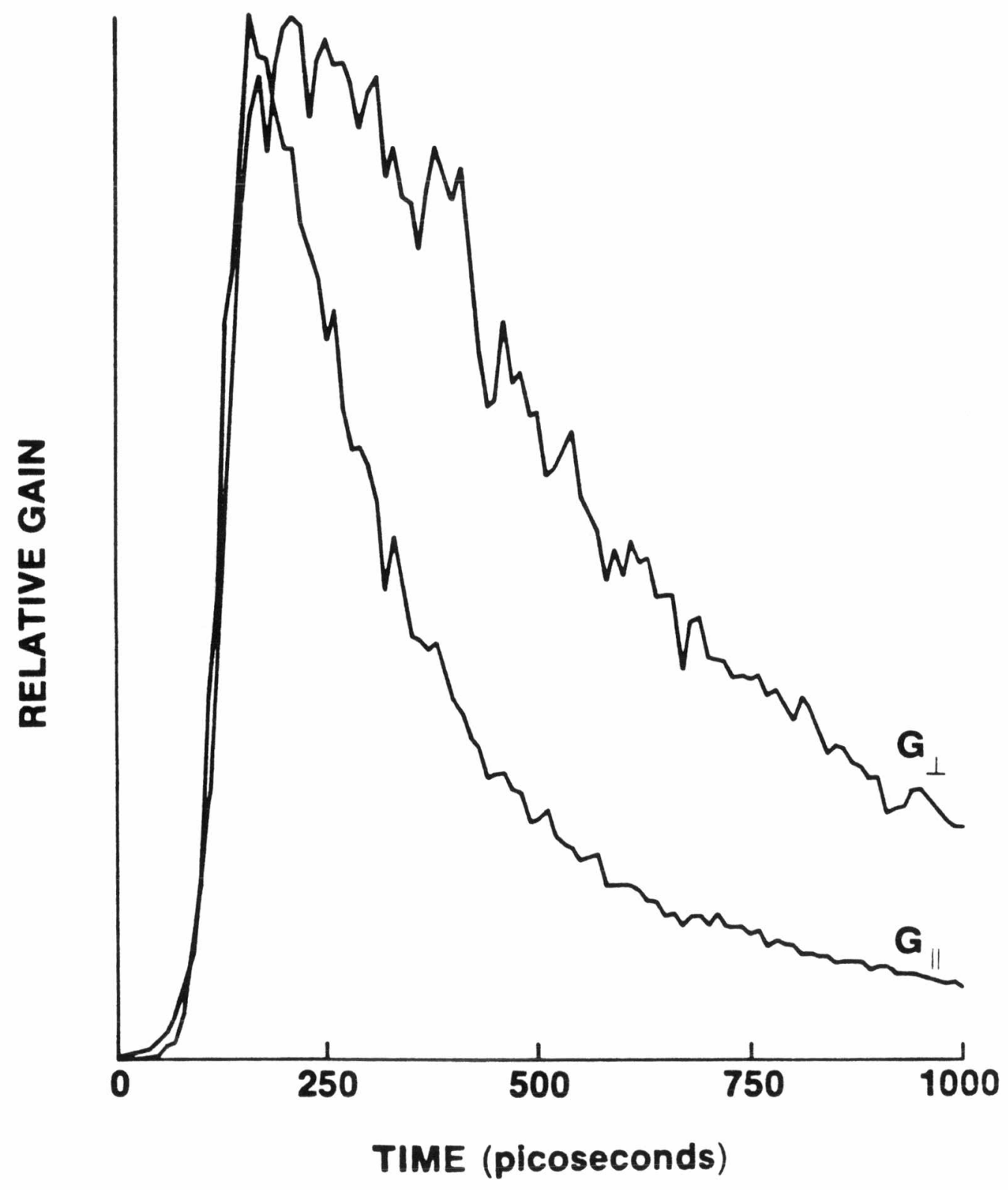

Figure 3-4 Polarization dependence of the gain storage time 
probe pulse is amplified, it too is sensitive to the cosine ${ }^{2}$ of the angle of the molecules relative to its polarization. Thus, the pump will excite some molecules that will give rise to gain even in the perpendicular direction. This accounts for the similarity in rise times.

The fall of the $G_{\|}$curve is due to fluorescence and reorientation. The $G_{\perp}$ curve has a longer decay which is due to two effects. First since the peak gain for the curve is less, the fluorescence decay is effectively a slower loss as described in section III.C.1. Fluorescence decay, however, does not account for the entire lengthening because the decay of the $G_{\perp}$ curve is slower than that observed for a $G_{\|}$curve of the same gain. The lengthening is due instead to the fact that more excited molecules are likely to rotate towards the perpendicular direction than away from it. The increase occurs because fewer molecules were aligned in the perpendicular direction originally. In conclusion, the difference between the peak gains $G_{\perp}$ and G $\|$ and the lengthening of the decay of $G_{\perp}$ are indicative of molecular reorientation.

II.C.3 Amplified Spontaneous Emission

Finally, the most interesting and potentially the fastest loss mechanism is amplified spontaneous emission. The light that constitutes fluorescence is spontaneous emission and is emitted into all directions. A portion of 
the spontaneously emitted light will traverse the region of the dye cell that is pumped. The light is then amplified at the small signal gain rate generating amplified spontaneous emission (ASE) which can grow quite large and remove a large portion of the energy stored in the amplifier. The quantity of $\mathrm{ASE}$ is nonlinear with the gain.(73) As the gain increases, so does the quantity of spontaneous emission as well as the amplification factor. Thus ASE is of particular importance in high gain amplifiers. (74) Results will be presented in section III.D that quantify the loss rate.

The ASE is not just a loss mechanism but is also a noise source. ASE noise must be prevented from propagating down the amplifier chain and dwarfing the signal. To this end, the amplifier stages must be isolated from each other in most systems. Since we pump with 70 ps pulses, we need to excite the molecules only once; we do not have to recycle the population to maintain the gain. As a result the ASE is less severe as a noise source.

The effects of geometry on ASE will also be investigated. For this purpose $I$ will define the longitudinal ASE as that which propagates spatially coincident with the amplified beam. The longitudinal ASE is noise which is unavoidable even with spatial filtering. The transverse ASE includes both the ASE which propagates across the face of the amplifier as well as that ASE that 
propagates into the larger solid angle subtended by the gain region. Transverse ASE is clearly geometry dependent and will be investigated as such.

To quantify the decrease in gain due to the above loss mechanisms, the following procedure was used. First the log of the transient gain data was plotted as in Figure 3-5. Next a best fit straight line was drawn through the decaying portion. The best fit was done by the computer using a least squares method. The $1 / \mathrm{e}$ time for the decay was then tabulated and will be referred to as the gain storage time of the amplifier. For any particular set of dye and pump parameters, a pump-probe experiment can be performed and a single number assigned as the storage time of the amplifier.

II.D Transient Gain Results

A series of transient gain measurements were performed with different pump intensities, solvents and geometries in order to quantify the effects of the different loss mechanisms. The effect of varying dye concentration was also investigated and the results are presented in Appendix 2 .

III.D.I Solvent Dependence

The initial experiment involved measuring the storage time as a function of pump intensity. Care was taken to keep the pump and input pulse intensities below the level 


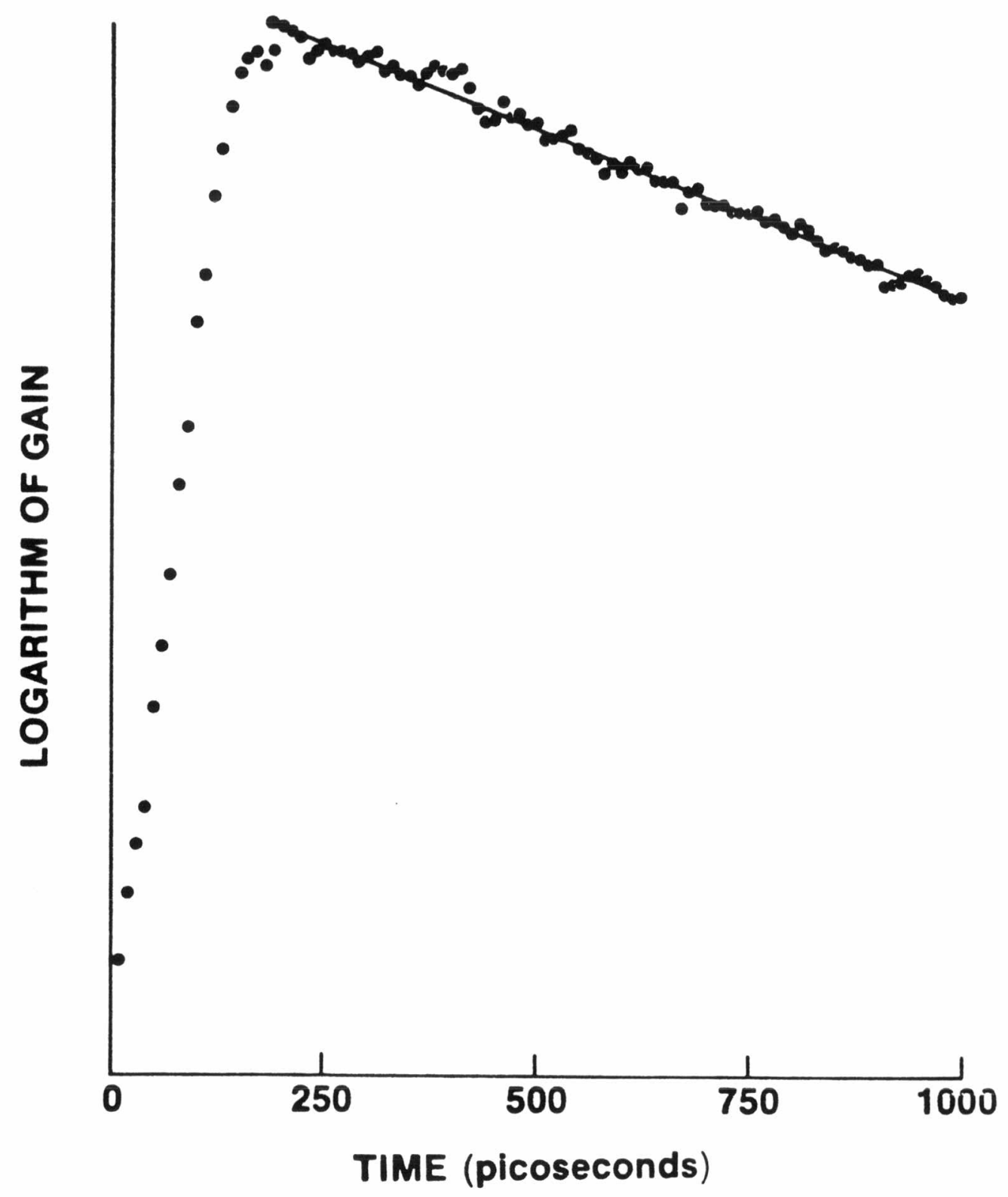

Figure 3-5 Log of data and best fit line 
where nonlinear index effects would dominate the results (see section II.D.15). The experiment was performed with two different solvents: water and ethylene glycol. The results are displayed in Figure 3-6. At the low pump and, hence, low gain limit the storage times for the two solvents differ greatly. The values do not approach a limit of $2.8 \mathrm{~ns}$ as would be expected for fluorescence decay. Instead the curves approach limits of 600 ps for ethylene glycol and 250 ps for water. We conclude that in the low pump and low gain regime, molecular reorientation is the dominant loss mechanism. The different results for the two solvents are due to the difference in viscosity and, hence, molecular reorientation time. (75) For a similar dye, rhodamine 6G in water, Eichler(76) gives a value of $160 \mathrm{ps}$ for the molecular reorientation while Podolski(77) reports 210 ps. For ethylene glycol values range from 1 ns measured by Lessing (78) up to $2.1 \mathrm{~ns}$ reported by chuang and Eisenthal. (79) our results for water show that for low viscosity solvents the low gain storage time is the same as the molecular reorientation time. The gain storage time in Ethylene glycol is somewhat shorter than the molecular reorientation time because the fluorescence also contributes.

Glycerol, with a viscosity higher than ethylene glycol was also tried as a solvent; however, sample preparation problems (bubbles, water) precluded an accurate 


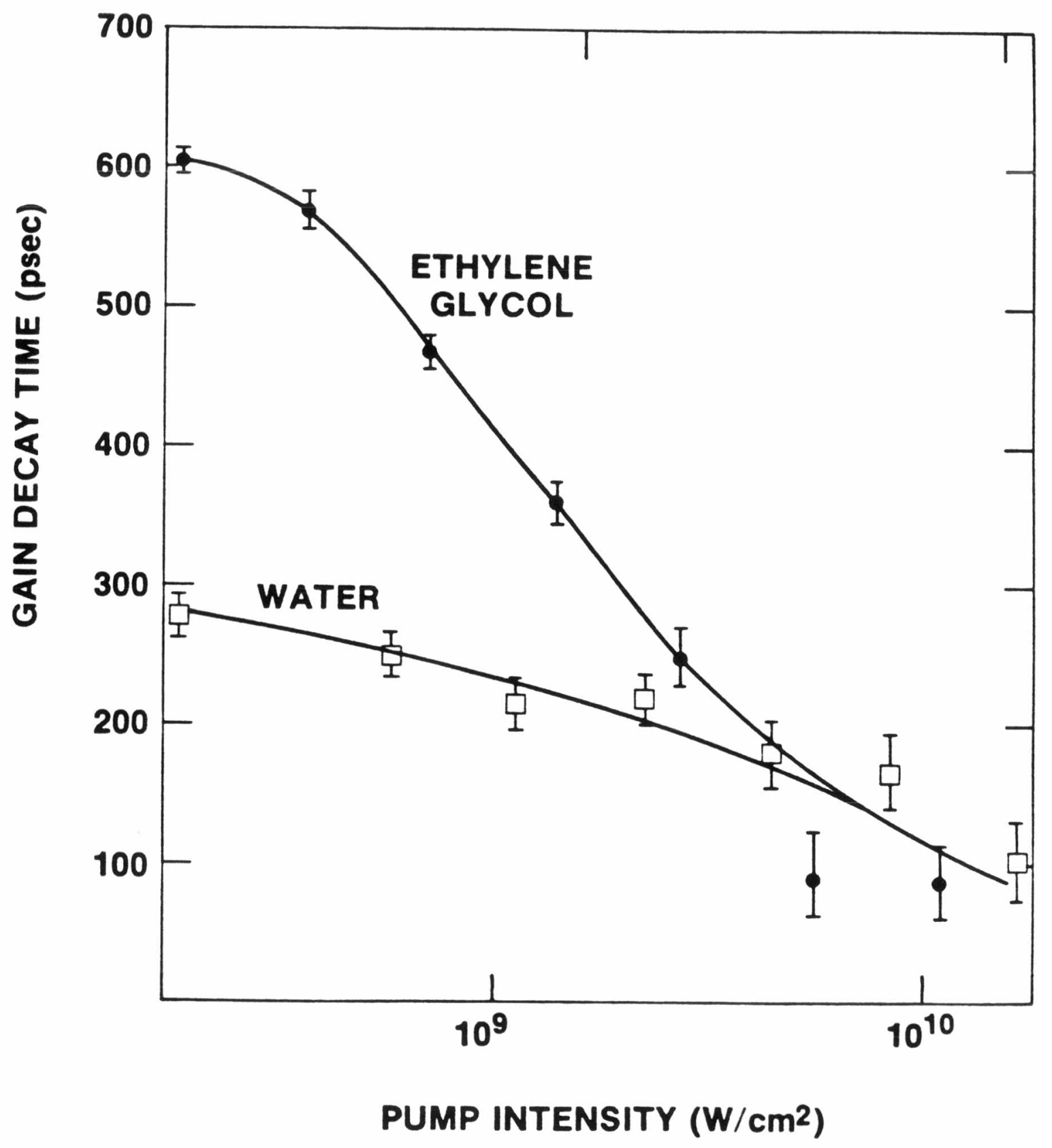

Figure 3-6 Solvent dependence of the gain storage time 
determination of the gain storage time.

Referring again to Figure $3-6$, a clear trend can be observed. For both solvents, the gain storage time is decreased with increasing pump energy and hence increasing gain. The loss mechanisms that dominate in the high gain regime are fluorescence and ASE. The peak gain measured for the water experiment is displayed in Figure 3-7 as a function of pump intensity. The data is used to convert the pump intensity axis in Figure $3-6$ to a gain axis. The results are plotted in Figure 3-8 and show the predicted shortening of the gain storage time (from Figure 3-3) and the measured gain storage time (from Figure 3-6) as a function of gain. The upper theoretical curve includes only the effects of fluorescence in the calculation. The difference between the two curves in the high gain regime is due to ASE. Molecular reorientation plays little role here as evidenced by the convergence of the water and ethylene glycol curves in Figure $3-6$.

In summary, molecular reorientation is the dominant loss mechanism in the low gain limit. In the intermediate regime, molecular reorientation, fluorescence and ASE all play a part and the storage time decreases significantly. Finally in the high gain limit, fluorescence is important but $\mathrm{ASE}$ is the dominant loss mechanism as the storage time shortens even further. Above a pumping intensity of 


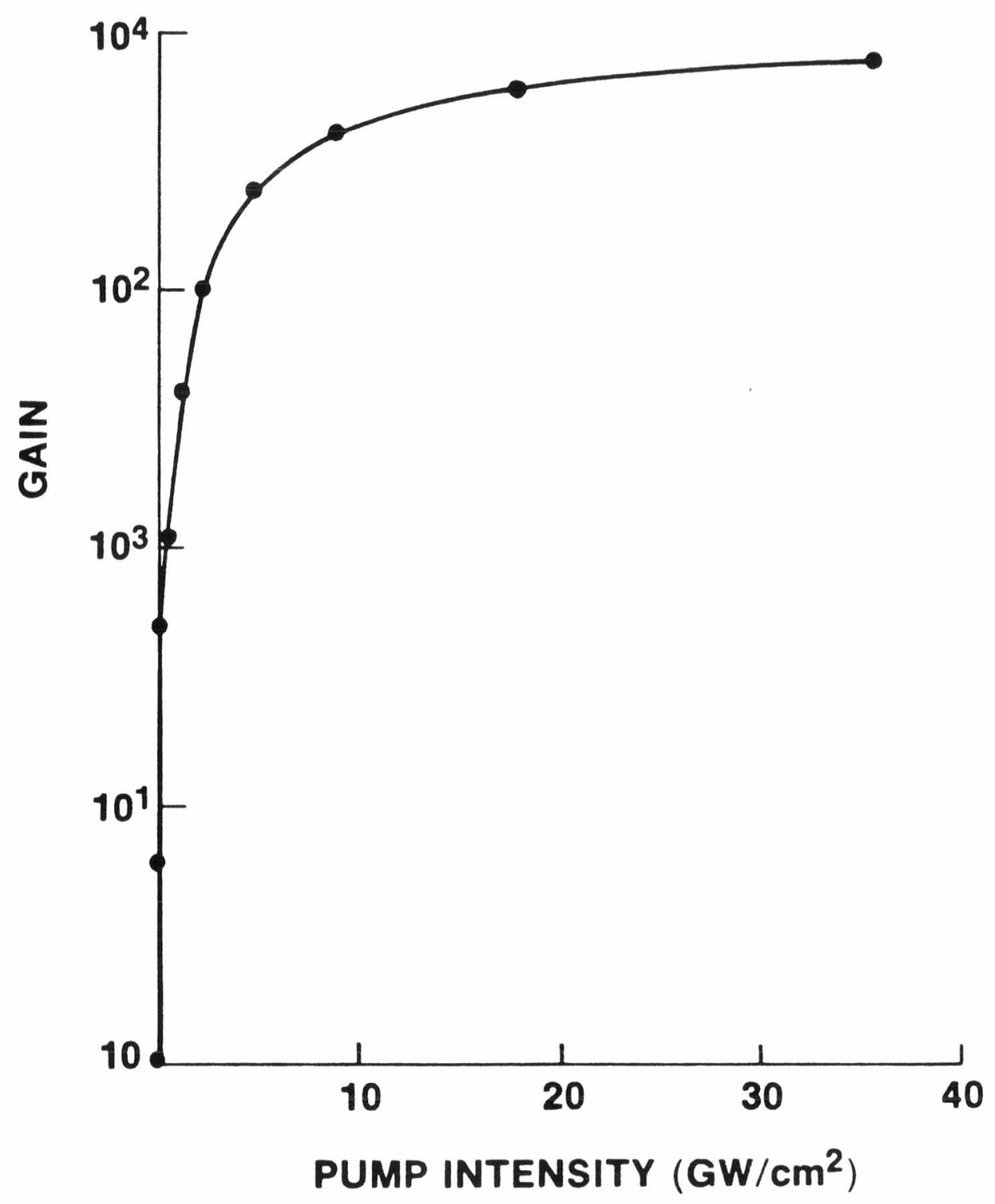

Figure 3-7 Pump intensity dependence of the peak gain 


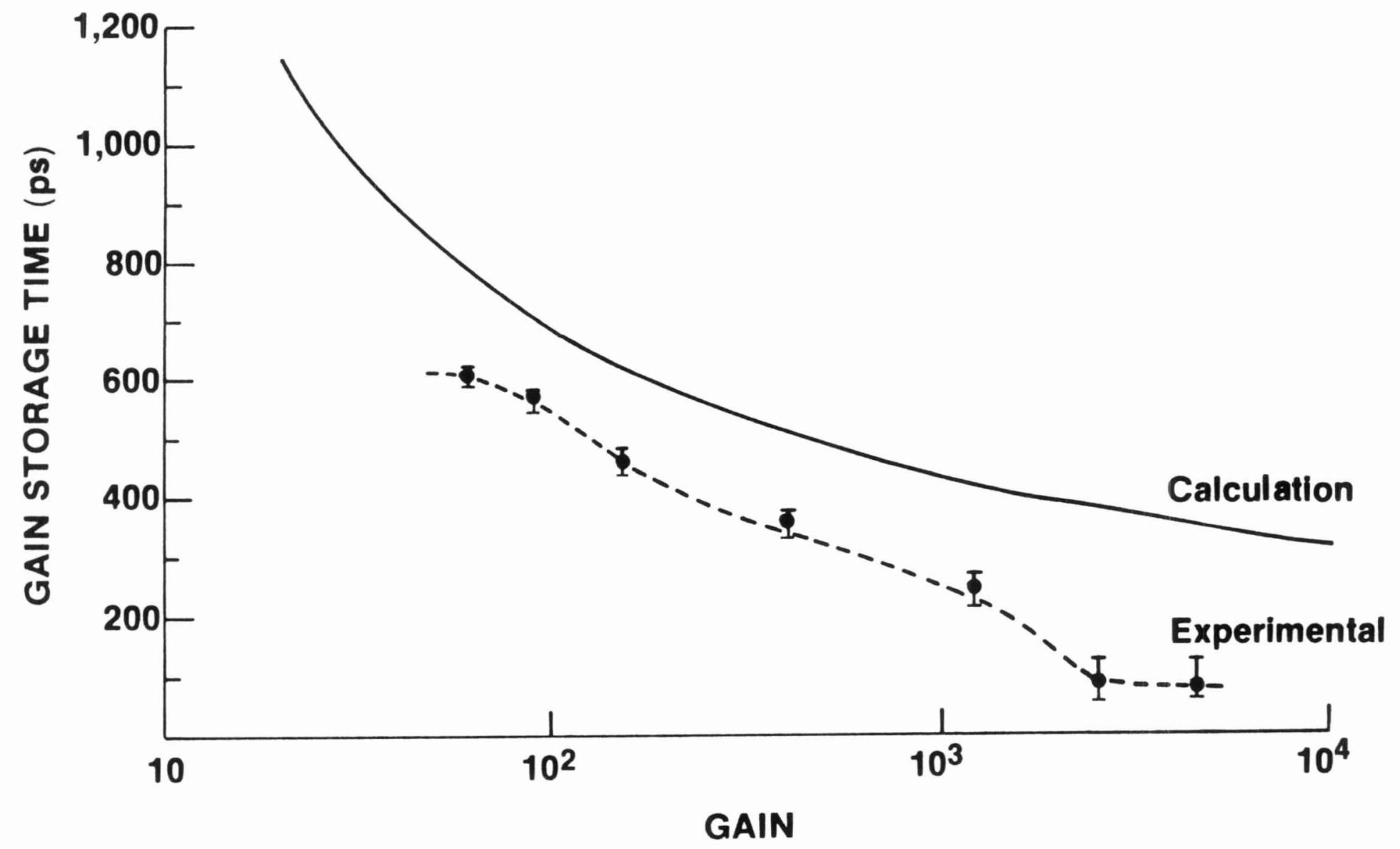

Figure 3-8 Comparison of gain storage time calculation and experiment 
$10^{11} \mathrm{~W} / \mathrm{cm}^{2}$, nonlinear effects in the solvent and damage to the glass dye cell prevent any further measurements.

\section{III.D.2 Spot Size Dependence}

A second experiment was performed in which the pump intensity was varied and the storage time measured. The pumping geometry was changed by varying the spot size of the active region. Even though the pump beam spatial profile is gaussian, a spatial cross section of the gain region is super-gaussian or even roughly rectangular because the amplifier is saturated in the central region. In the following arguments, a rectangular gain profile is assumed. The transient gain was measured for a $30 \mu \mathrm{m}$ spot and then a $60 \mu \mathrm{m}$ spot. For the larger spot, with 4 times the area, 4 times the pump energy was used. In this manner the energy on any given area, and hence the intensity $\left(w / \mathrm{cm}^{2}\right)$, was the same. The gain along any ray parallel to the pump should be the same in both cases. The only difference between the two is that the active area has been enlarged in the $60 \mathrm{~m}$ spot.

A word about the accuracy of the spot sizes is in order. The spot sizes were calculated using gaussian beam theory $(80)$ and measurements of the beam diameters at the focussing lens. Two lenses with focal lengths of $74 \mathrm{~mm}$ and $166 \mathrm{~mm}$ were used. Thus, while the exact values of the spot sizes are not known, the ratio of the sizes is. The ratio of the energies required to obtain equal intensity can then 
be easily achieved.

The results of the above experiment are shown in Figure 3-9. The measurements were performed with water as the solvent and so the low gain storage time is $250 \mathrm{ps}$ regardless of geometry. Again, as the pump increases, the storage time decreases. For the larger spot the storage time decreases more quickly and eventually to a lower value. The minimum storage time observed was 35 ps but only for the larger spot.

The effect on the gain of longitudinal ASE, that which overlaps the amplified beam, is the same for both spot sizes. The transverse ASE, however, is greater for the larger spot. Because the active region subtends a larger solid angle, we conclude that it is the transverse ASE that accounts for the difference between the two curves. It is also the transverse ASE that will limit the gain in larger diameter amplifiers because of the extremely short storage times. Note that not all of the energy contained in a $70 \mathrm{ps}$ pump pulse can be deposited in the amplifier when the losses due to ASE are as fast, or faster, than the pump pulse.

II.E Scaling

Considering the various loss mechanisms, conclusions can be drawn for scaling up to higher energy amplifiers. The spot size is the most important parameter and will 


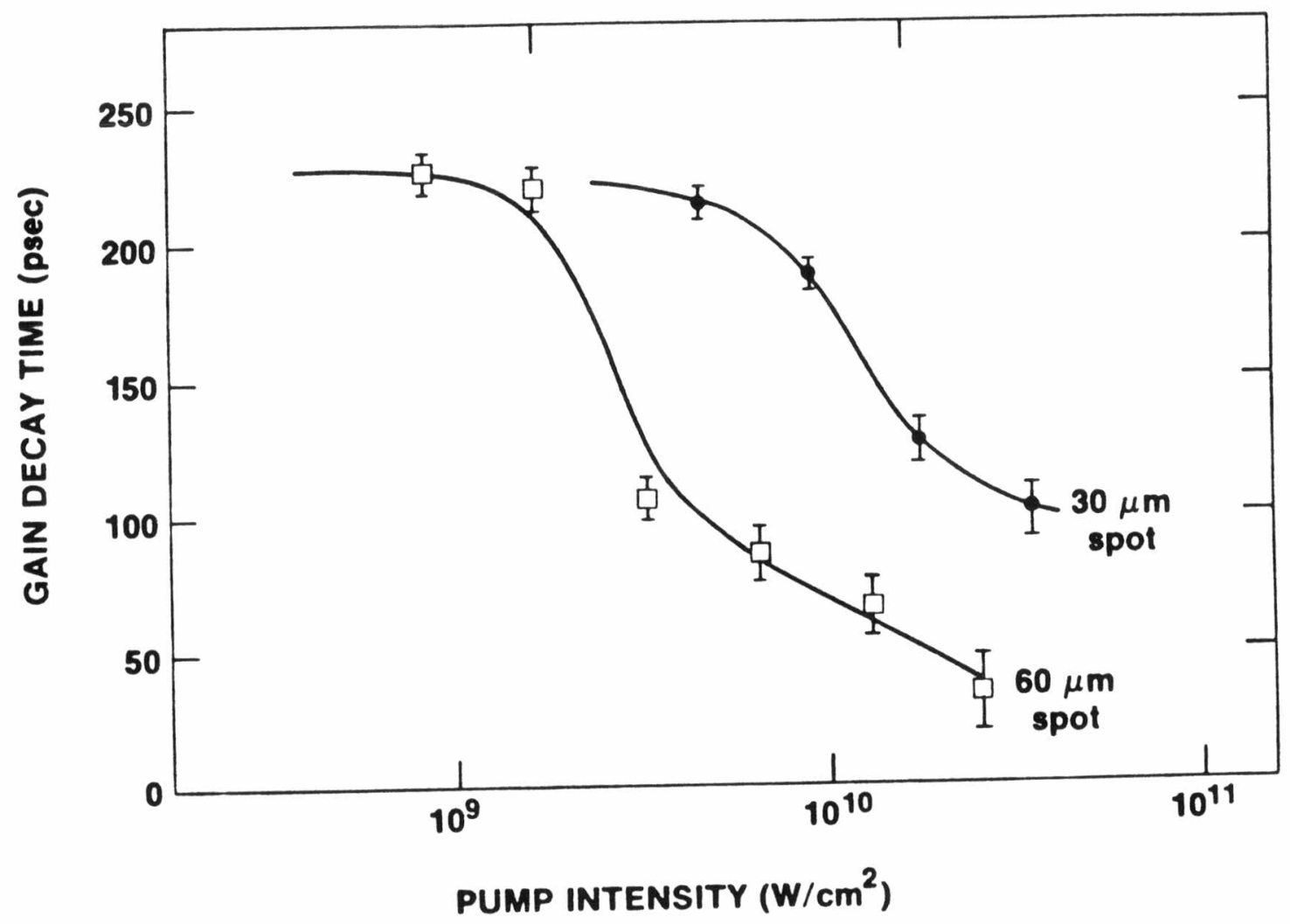

Figure 3-9 Spot size dependence of the gain storage time 
determine what the input intensity is and how far into saturation the amplifier is run. Recall from section II.D.3 that for an amplifier to be efficient it must be run above saturation. To preserve the temporal shape of the input pulse, however, the amplifier must be run in a linear mode or below saturation. The saturation level is reached when half the excited molecules have been removed from the excited state. At the saturation level, the gain is the square root of the small signal gain (Equation 3-4). The compromise between pulse broadening and efficiency is reached by running the amplifier so that the output pulse is approximately at the saturation level. The intensity of the output pulse is adjusted by changing the spot size in the amplifier. Thus, for a given output energy level, the diameter of the amplifier is fixed.

For example, if an amplifier system was designed to operate with 10 Joules of pump energy in a short pulse then, assuming a conversion efficiency of 38 , an output pulse energy of $300 \mathrm{~mJ}$ would not be unreasonable. The peak power of the output pulse would then be above a terawatt. With a saturation level of $1.4 \mathrm{~mJ} / \mathrm{cm}^{2}$, the final amplifier should be approximately $10 \mathrm{~cm}$ in diameter. Luckily, dye solutions are inexpensive compared to solid state laser materials and a $10 \mathrm{~cm}$ dye cell could easily be fabricated. The laser to produce such a pump pulse now exists at the Laboratory for Laser Energetics. (81) 
The primary loss mechanism in a large diameter amplifier will be transverse ASE. Two solutions to the ASE problem present themselves. First, the amplifier could be segmented to prevent the ASE from propagating into the full solid angle subtended by the amplifier. segmentation would decrease the amount of ASE but might cause beam quality problems in the far field. The second solution is to use synchronous pumping. The time of flight across the face of a $10 \mathrm{~cm}$ amplifier is $500 \mathrm{ps}$. From the results of this chapter it is clear that the complete amplification process is over in $100 \mathrm{ps}$. Thus, one edge of the amplifier never sees the ASE from the other edge. Time of flight ASE suppression has never been observed because a system of sufficient energy has not yet been constructed.

Using short synchronized pump pulses will not only reduce the losses due to transverse ASE but will nearly eliminate the losses due to longitudinal ASE. The losses due to transverse ASE are very rapid; however, the losses due to longitudinal ASE are slower than the 70 ps pumping time, as can be seen from the minimum storage time of 100 ps in Figure 3-6. Finally, synchronous amplification is fast enough to avoid losses caused by molecular reorientation. 


\section{III.F Saturation}

All of the subpicosecond dye amplifier systems that reach the $1 \mathrm{~mJ}$ output level have contained three or four amplifier stages. As discussed in section II.D.2 the gain in any single stage is limited by ASE and saturation. To achieve an overall gain of $10^{6}$ or more it is necessary to use multiple stages. (35) The stages must usually be isolated from each other to prevent ASE from propagating down the amplifier chain. Each stage has its own set of design criteria. There is a minimum spot size in each amplifier in order to keep the subpicosecond output pulse intensity below the saturation level so that the pulse will not broaden. Once the spot size is chosen, the pump and input pulse intensities are restricted to avoid nonlinear index effects which will degrade the beam quality (see Section II.D.15)

If the quantity of pump energy was unlimited, a system could be constructed with all the amplifiers identical. The spot size would be increased in the earlier stages to the size required to keep the last stage below saturation. Extra pump energy would be required but such a system would be easier to align. This philosophy is currently being used in a $20 \mathrm{~mJ}$ system being constructed at the Laboratory for Laser Energetics. (82) We are concerned with building an efficient system, however, and it is unnecessary to pump the 
large spot sizes in the earlier stages when smaller spots are acceptable. For this reason we have built a three stage system where the parameters for each stage are different.

III.F.1 Comparison of Stages

Many quantities will be the same in each stage. We have chosen to use longitudinal pumping throughout and, as a result, the same dye is used in each stage. We have chosen to use the same length for each stage and, as a consequence, the dye concentrations are similar. In addition, the solvent, dye flow system and system for timing each stage are the same. The input pulse intensity, however, must increase with each stage. For an efficient system, the spot size and the pump intensity must increase correspondingly. It is these three quantities that differentiate the three stages.

\section{III.F.2 Pump and Input Saturation}

Two types of saturation will be dealt with in the remainder of this chapter: saturation of the amplifier due to the input pulse and saturation due to the pump pulse. The physical interpretation for amplifier saturation by the input pulse is that after the pulse has been amplified only half the excited molecules are left in the gain medium. (83) Thus if the input pulse removes one half of the stored energy the amplifier is said to be saturated. It is in the 
saturated regime that the amplifier is most efficient and that the pulse shape is distorted. Saturation due to the pump pulse is similar. The amplifier is saturated by the pump when more than half the ground state molecules are pumped into an excited state. It is at saturation that the pump is said to bleach the dye and the transmission of the pump increases. In section III.F.4 and III.F.5 I will present curves that describe the saturation of the amplifier due to both the pump and input in each stage.

\section{III.F.3 Apparatus}

A photodiode with an oscilloscope or the LSI-II computer for averaging was used to measure the gain in each amplifier stage. Initially, the first stage was optimized and the diode placed immediately after it. The pump intensity was then reduced by factors of two using wratten plastic neutral density filters. Because these filters are thin, no realignment of the system was required. The gain was then recorded over a large range of pump energies. Next, the pump was restored to its maximum intensity and the input pulse intensity was decreased by factors of two. The CW beam from the dye oscillator caused thermal effects in the plastic filters and schott glass filters were required. The alignment of the amplifier was checked after any change in the filter stack because the $2 \mathrm{~mm}$ thick glass filters did misalign the input beam. 
Next, the first stage was run with the maximum input and pump energy and the diode was placed after the second stage. The gain was then measured with attenuation placed only in the second stage pump beam. Likewise, the gain was measured with the input beam being attenuated after the first stage but before the second stage. Finally, with the first two stages operated at maximum pump and input energies the gain of the third stage was measured. As before the pump and input intensities were attenuated only for the third stage and the gain was recorded.

Calibration of the actual input pulse energies was accomplished using the high repetition rate beam from the dye oscillator. First the power of the input beam was measured at the oscillator and immediately after each stage. These values are given in Figure 3-10 and can be used to calculate the transmision of each element in the system. The input power is converted to energy per pulse using a repetition rate of $10^{8} \mathrm{~Hz}$. The ratio of the amplified pulse energy to the input pulse energy is measured by a photodiode and yields the gain.

\section{III.F.4 Pump Energy Results}

The gain measurements versus pump energy for the three stages are given in Figures $3-11,3-12$ and $3-13$. Each of the curves is plotted with a log axis for the gain and a linear axis for the pump. For every stage, the gain 


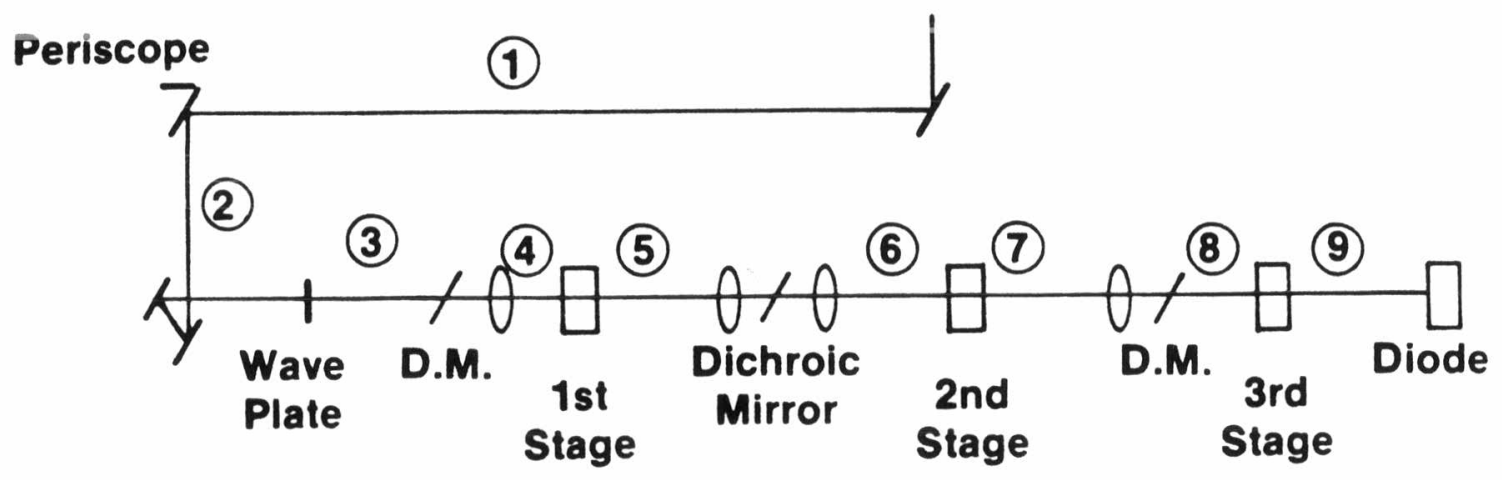
(1) $26 \mathrm{mw}$
(2) $25 \mathrm{mw}$
(3) $21.5 \mathrm{mw}$
(4) $20.5 \mathrm{mw}$
(5) $7.5 \mathrm{mw}$
(6) $5.5 \mathrm{mw}$
(7) $2.5 \mathrm{mw}$
(8) $2.0 \mathrm{mw}$
(9) $1.6 \mathrm{mw}$

Figure 3-10 Dye amplifier chain transmission 


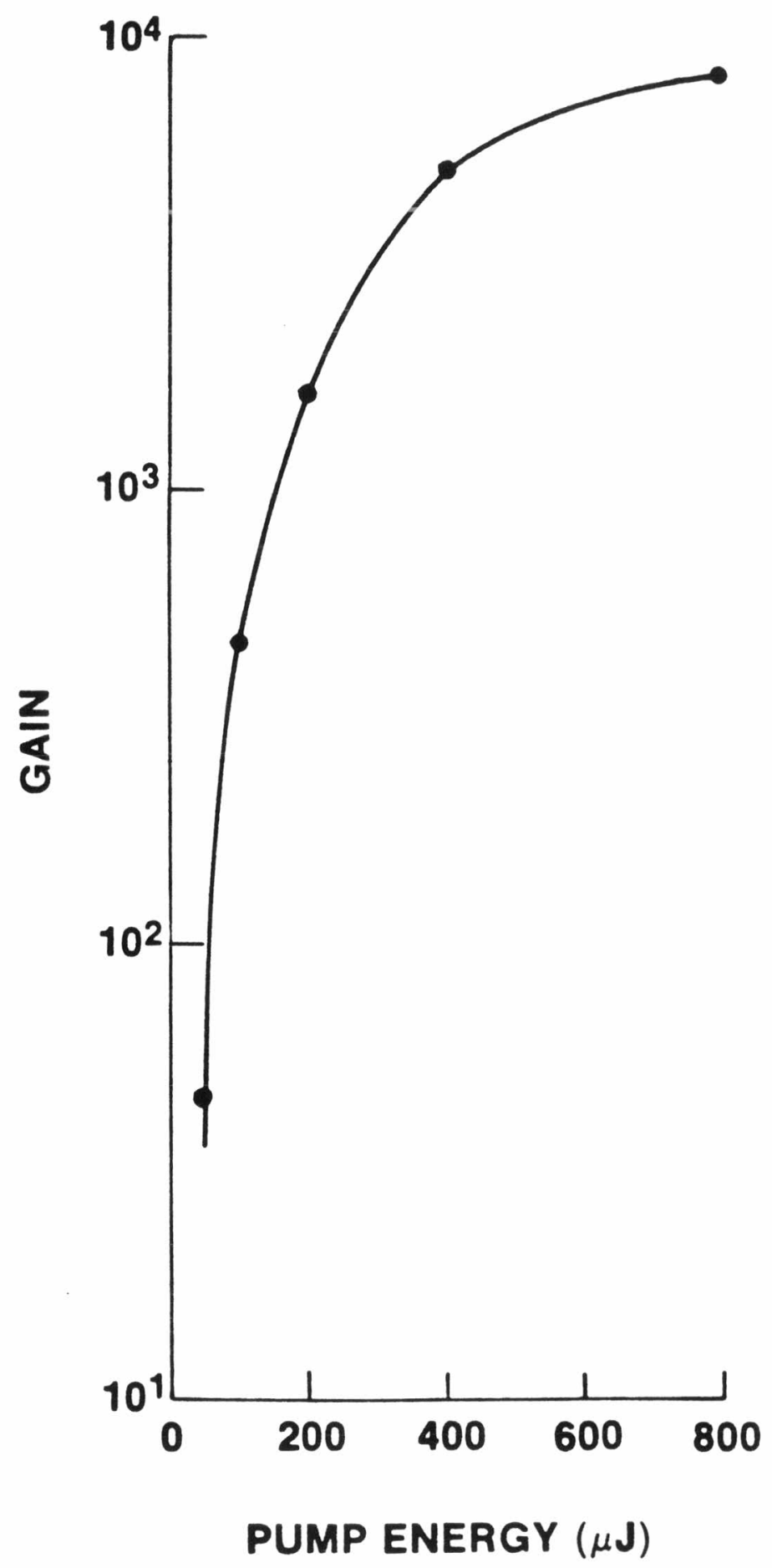

Figure 3-11 Pump intensity dependence of the first stage peak gain 


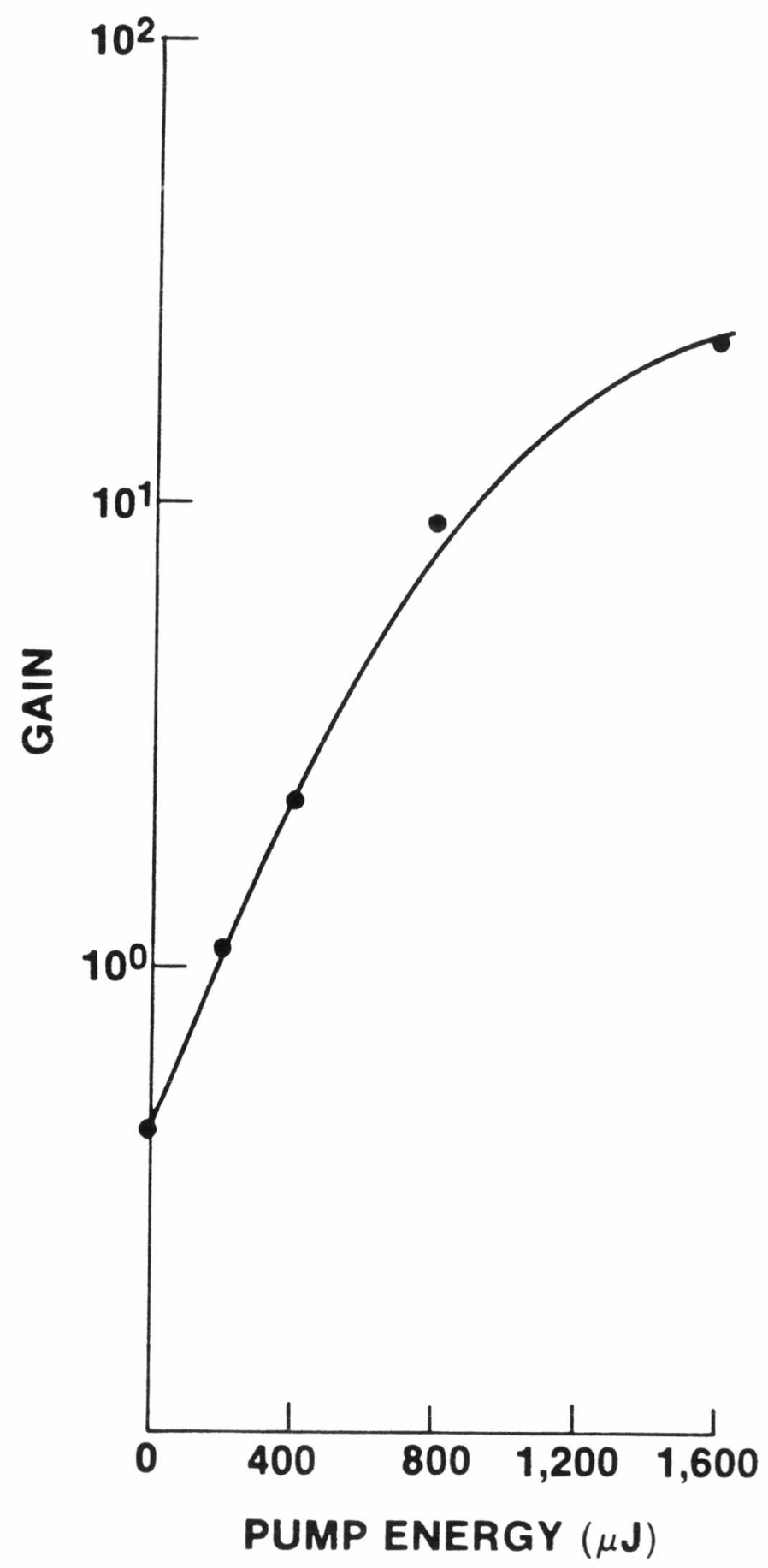

Figure 3-12 Pump intensity dependence of the second stage peak gain 


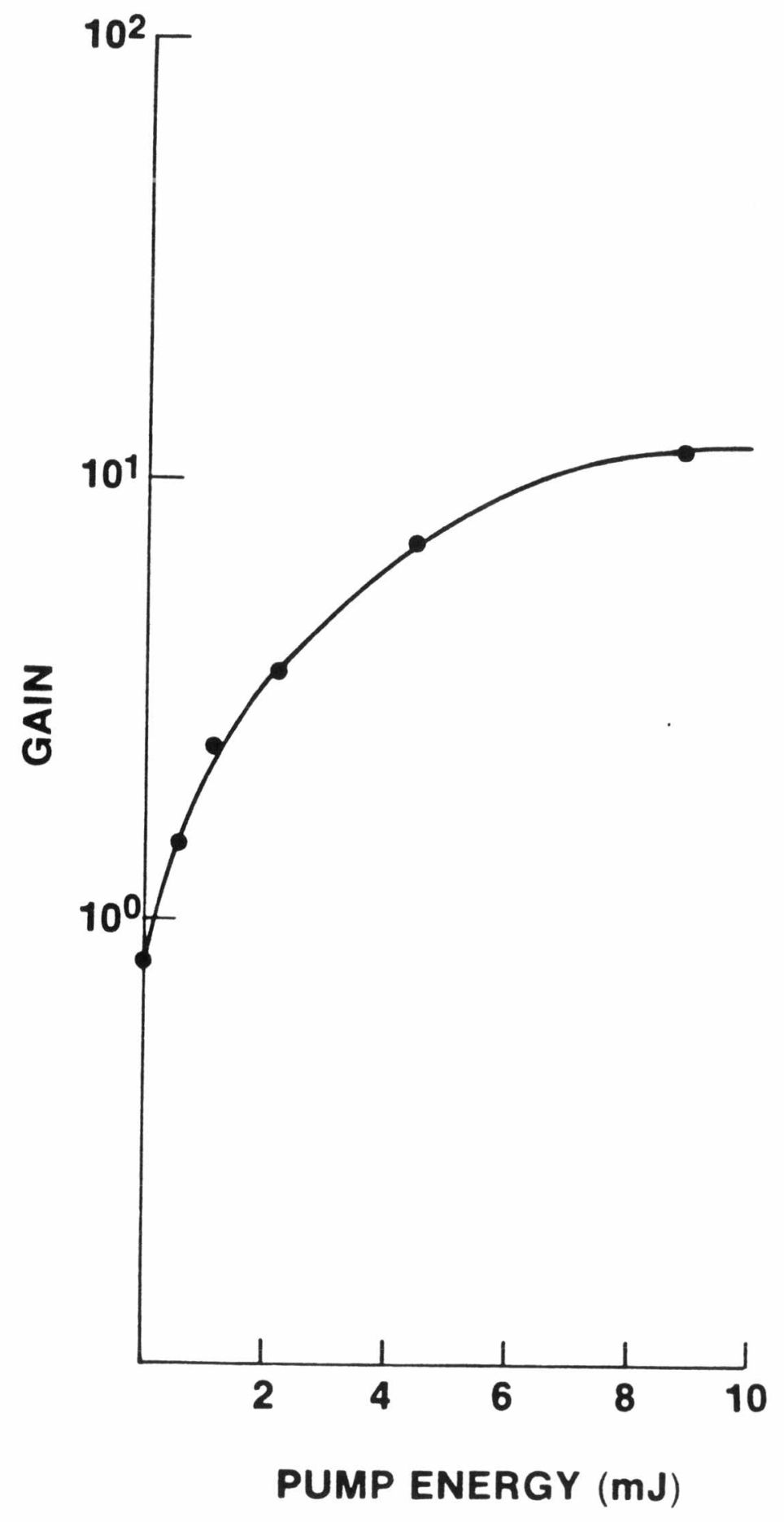

Figure 3-13 Pump intensity dependence of the third stage peak gain 
increases exponentially with increasing pump energy in the low gain limit. Eventually, the pump saturates the amplifier and the gain increases more slowly. It will be shown theoretically in Chapter IV that this trend is true for all amplifiers. The deviation from a straight line indicates the degree of saturation.

The pump energy increases in the later stages and the peak gain decreases which is true of all efficient amplifier systems. The increase in pump energy is required because of the increase in spot size in the later stages in order to keep a constant pump intensity. Although the gain in a particular stage can vary somewhat from day to day (see Section II.D.13), the total system gain will change little because of saturation. The gain versus pump energy curves were measured several times over a period of a few weeks. The results presented here represent the best signal to noise measurements. The peak gain for the second stage is typically higher than in Figure 3-15. Quantities that may vary include the beam divergence, energy, pulsewidth and wavelength of the dye oscillator as well as the divergence and energy of the pump pulse.

These gain versus pump energy curves were measured to show the role saturation played in each amplifier stage. Subsequent to these measurements, the spot sizes of the stages were enlarged to move further from saturation and 
prevent pulse broadening. These curves also display the major differences between the three stages: the gain decreases and the pump energy increases in the later stages.

III.F.5 Input Energy Results

In Figures $3-14,3-15$ and $3-16$ are the gain versus input energy curves. These curves are plotted with a log axis for the gain and a log axis for the input. For each stage, the gain is greatest at the lowest input level and decreases as the input energy is increased. In the second stage curve, it can be seen that there appears to be a low input limit to the gain. The low input energy limit will be shown to be theoretically correct in Chapter IV. This limit is known as the small signal gain ${ }^{(84)}$ and occurs when the input pulse essentually does not deplete the energy stored in the amplifier at all. As the input energy increases the number of excited state molecules will begin to be reduced and, hence, the gain will decrease. In the limit of heavy saturation the gain decreases to 1 .

The daily operating point for the amplifier stages corresponds to the highest input level in the figures. The degree to which the gain is less than the small signal gain is indicative of saturation. Recall from section III.E that the saturation level is reached when the gain is the square root of the small signal gain. Using this criteria, it can be seen from the figures that each of the amplifier stages 


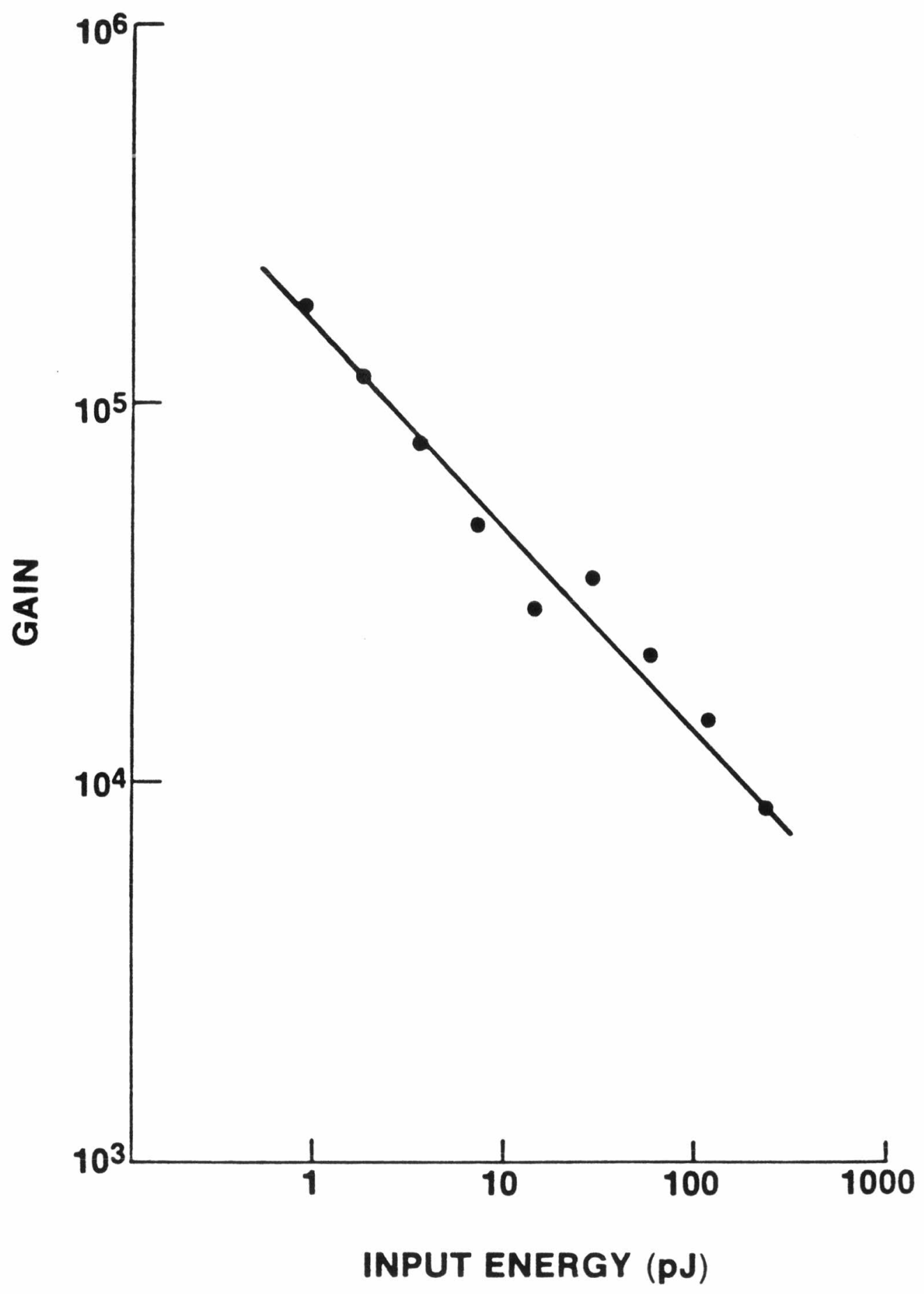

Figure 3-14 Input intensity dependence of the first stage peak gain 


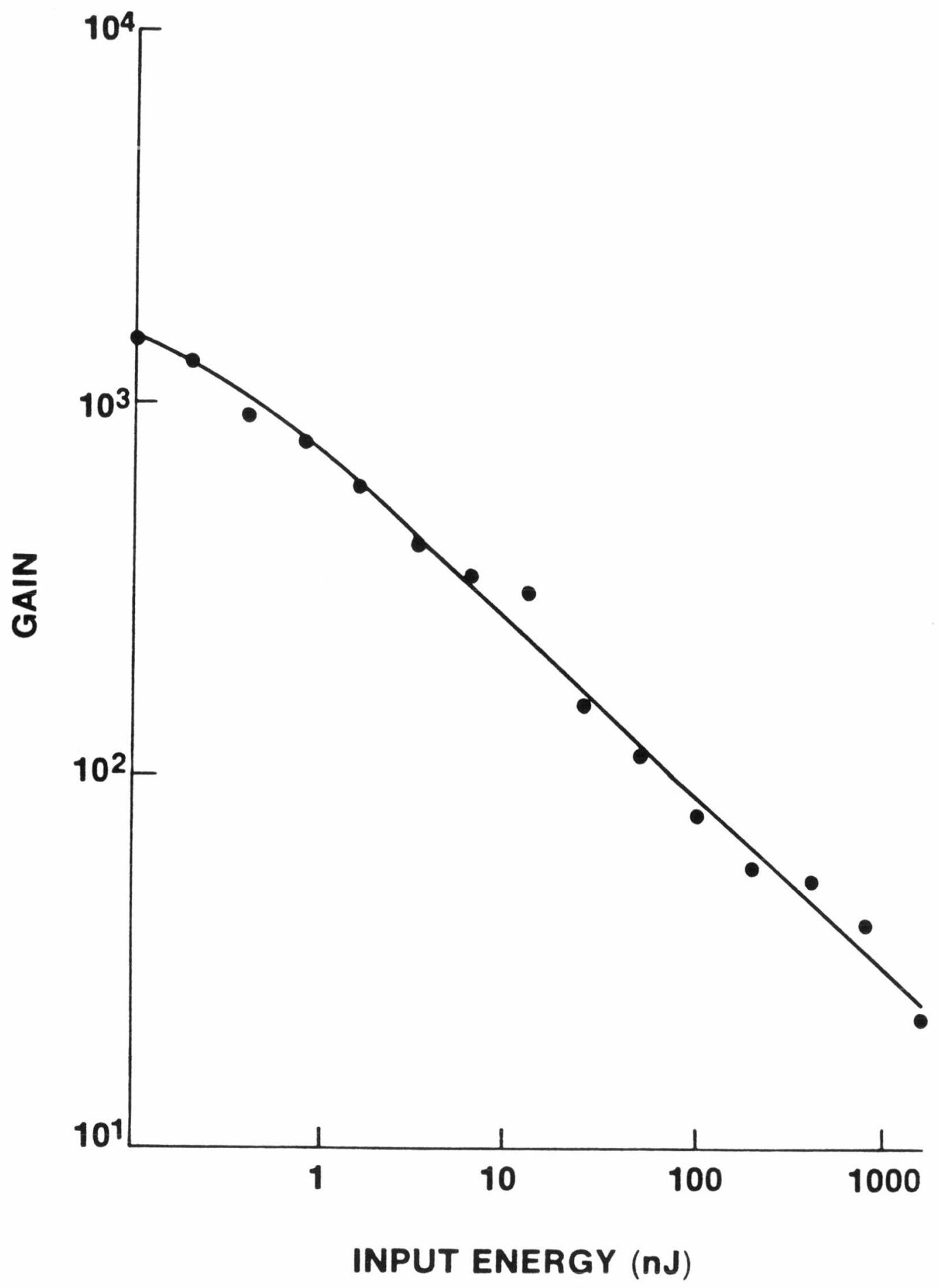

Figure 3-15 Input intensity dependence of the second stage peak gain 


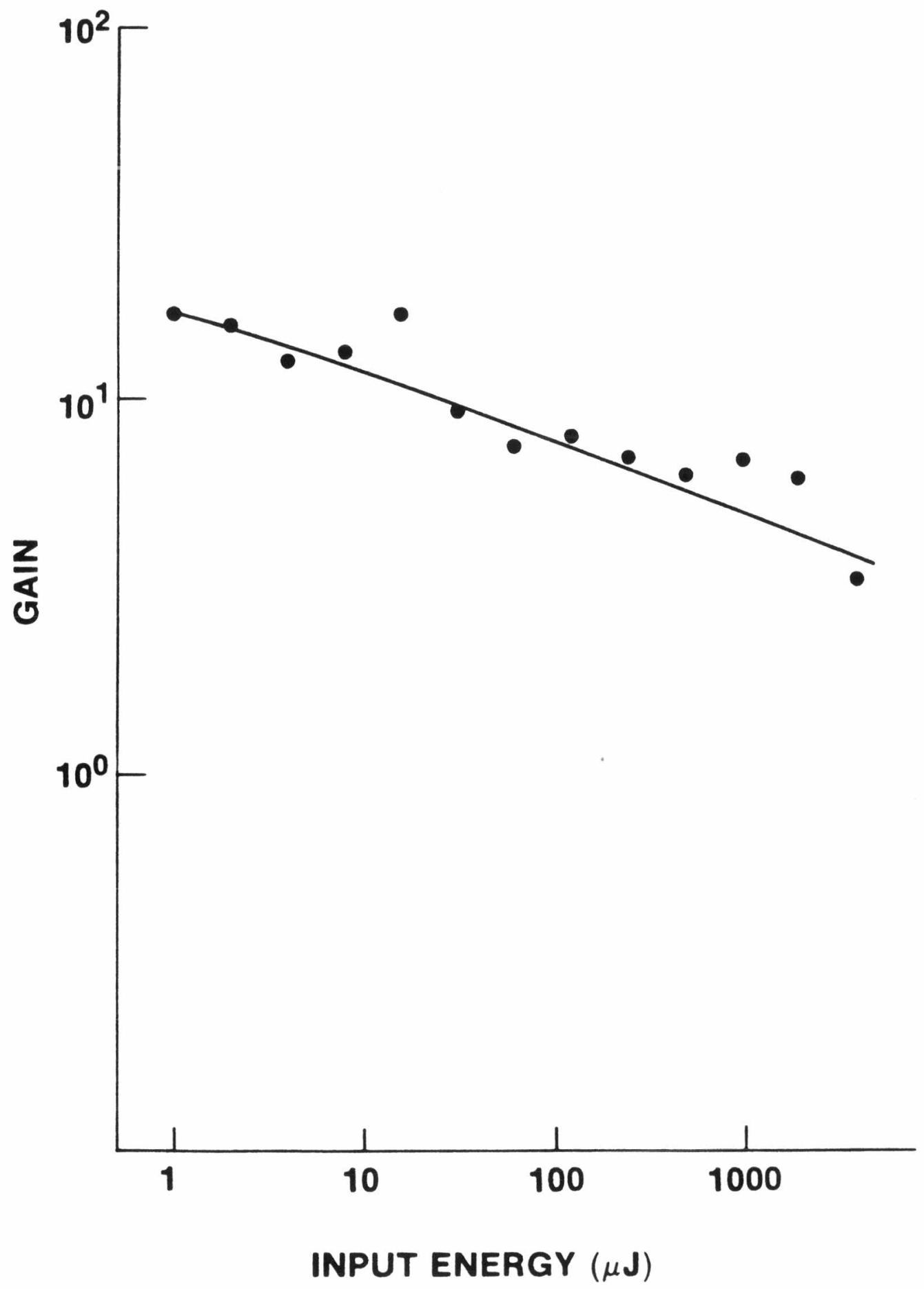

Figure 3-16 Input intensity dependence of the third stage peak gain 
is operated somewhat above saturation. The same cautions concerning the pump curves should be applied to the input data also. The differences between the stages can be summarized as follows: the gain decreases and the input energy level increases in the later stages.

\section{III.G Conclusion}

In this chapter, I have presented the results of several experiments performed on the dye amplifier system. The first part of the chapter dealt with results common to all stages of the dye amplifier system. The remainder of the experiments illuminated the differences between the stages. The goal of the study was to investigate the efficiency of an amplifier system pumped by short synchronized pump pulses. In addition we wish to understand the physics behind the dye amplifiers. Such an understanding is a prerequisite for optimizing the system.

Optimization requires a knowledge of what parameters may be changed. The effects of different pump and input intensities, pumping geometry, solvent and dye concentration have been addressed. These are exactly the experimental quantities that a designer must consider when building a dye amplifier system. By using these same parameters as independent variables in the experiments it is hoped that the results will be directly applicable to system design problems. 
The main consideration in designing a high efficiency amplifier is defining the limits of the gain. In Chapter III, I have identified the two major limits as fast loss mechanisms and saturation. The trade-offs inherent in saturation were discussed and results for all three stages presented. The loss mechanisms present no such trade-offs. Fluorescence, molecular reorientation and ASE were investigated as the dominant loss mechanisms and the importance of each was discussed. The present design, using short synchronized pump pulses, was shown to be superior for reducing the effects of these losses. 
CHAPTER IV

THEORY

Steady state amplifier theories are not adequate to model our system, since we pump the dye amplifiers with very short pump pulses. In this chapter, I will present an expanded theory for modeling dye amplifiers, which is referred to as a time resolved theory, because no steady state assumption is needed. The time resolved theory will be used both to model the experimental results of Chapter III and to predict quantities that are difficult to measure experimentally. Initially, a review of the literature is presented. The need for a new theory to cover the short pump pulse regime is highlighted. The model is then presented and the method of solution is detailed. Comparisons are made to the experimental results of Chapter III. Both the transient gain and the saturation of the amplifier are investigated. Next, the case of short pump pulses is compared to longer pump pulses. Finally, the effects of amplifier saturation on pulse shape are analyzed.

IV.A Literature Review

A large number of theories for explaining laser amplifiers have been published over the last two decades. (85) All of the dye amplifier theories invoke a 
steady state gain assumption. The steady state theories assume that for a pump pulse with a duration longer than the fluorescence decay time of the dye $(\tau)$, and no input pulse, the gain will reach a constant value. We wish to model the case of a short pump pulse in which the gain never reaches a steady state value (see Figure 3-2). None the less, a review of previous theories is in order.

IV.A.I Short Pulse Amplifiers

The case where the input pulse is shorter than the fluorescence decay time of the dye $(\tau)$ was treated for the first time by Frantz and Nodvik. (86) In 1963 they analyzed a two level system and, using rate equations, calculated the amplified profile for a square and a lorentzian input pulse.

The Frantz-Nodvik formalism was used by New $(87,88)$ in two papers. He included different absorption and emission cross sections. The reshaping of the input pulse was calculated and the results were applied to the generation of ultrashort pulses in passively modelocked dye lasers.

This author has found two other papers both useful and eminently readable. The first is a consolidation of previous results by Trenholme and Manes. (84) These authors begin with the steady state approach and give dimensionless universal amplifier curves. In the latter portion of their report they deal with short pulse amplification and pulse 
reshaping. A second paper which deals only with the short pulse case was published recently by Frigo. (89) Frigo gives the most intuitive approach to the problem and the paper is a useful starting point for the reader. General properties are enunciated and applications are made to synchronously pumped oscillators and colliding pulse modelocking (CPM).

All of the above papers fall into one class. The papers assume a steady state inversion in the absence of an input pulse. If a short input pulse is included, the effects of interest include saturation and pulse reshaping. A second class of papers exists which deals explicitly with high power, multi-stage dye amplifier systems. The major issues are saturation and ASE.

IV.A. 2 Subpicosecond Gigawatt Amplifiers

Only two papers have been published that give a theoretical treatment of subpicosecond, gigawatt, dye amplifier systems. The first one published, and by far the easier to read, is by Migus, Shank, Ippen and Fork. (35) In their theory, ASE is included from the start as a mechanism competing for the gain. The formalism is based on the work of Ganiel, Hardy, Neumann and Treves (73) who first appreciated the significance of ASE in high gain dye amplifiers. Migus develops a two part theory. In the first part, the steady state excited population is determined by a balance between the generation of excited dye molecules by 
the pump and the depletion rate due to ASE. In the second part, amplification of the input pulse is calculated using an equation identical to that used in the papers mentioned above. The results are compared to an experiment which utilizes transversely pumped amplifiers and the stored energy predictions are accurate to 158 . The error in the stored energy corresponds to an error in the predicted gain of nearly a factor of 3 .

A similar system, but with longitudinally pumped amplifiers was modeled by Koch, Chiu and Yariv. (33) Their model begins with the general result for amplifying short pulses and then adds the effects of ASE as an approximation. The authors model the triplet state losses but do not include the higher lying states. The paper concludes with a section on optimization of amplifiers which is useful.

IV.A. 3 Transient Gain

One class of situations has not yet been investigated. This is the case where the input pulse is shorter than the fluorescence decay time of the dye and the pump pulse is shorter than the fluorescence decay time also. Our amplifier system operates in this regime. While some of the previous theories could be modified to include a gain that changes in time, to my knowledge only one prediction of the transient gain has been published. (33) No theory has been applied to the case of a pump pulse shorter than the 
fluorescence decay time $\boldsymbol{\tau}$.

As has been demonstrated in the previous chapter, we operate in a regime where the gain is not a steady state quantity. The delay time between the pump and input pulse is a very important parameter to be included. In addition to time resolution, the theory must include saturation, pulse shaping and ASE. No previous theory includes all of these effects as well as the ability to add additional energy levels to model the losses due to the higher lying states and triplet states. Finally, the theory I have constructed includes some geometrical information about the amplifier. A comparison of the major theories reviewed in the introduction is given in Table 4-1. The limitations of the time resolved theory are dealt with in section IV.B.3.

In the remainder of this chapter, I will describe the development and method of solution of the time resolved theory. General results for saturation are presented and compared to the data. Next, the transient gain measurements of Section III.D are modeled. The chapter concludes with theoretical predictions for different pump pulse durations and pulse broadening.

IV.B Time Resolved Amplifier Theory

A theory is sought that includes saturation of the amplifier and competition for gain with the ASE. In 


\begin{tabular}{r|c|c|c|c}
\multicolumn{2}{r}{ TRENHOLME } & MIGUS & KOCH & KAFKA \\
\hline \hline $\begin{array}{r}\text { Saturation } \\
\text { (pulse broadening) }\end{array}$ & YES & YES & YES & YES \\
\hline ASE & NO & YES & APPROX. & YES \\
\hline Triplets & NO & NO & YES & NO \\
\hline $\begin{array}{r}\text { Higher Lying } \\
\text { Excited States }\end{array}$ & NO & NO & NO & YES \\
\hline Time Resolution & NO & NO & SOME & YES \\
\hline Space Resolution & NO & SOME & NO & YES
\end{tabular}


addition, the theory must be time resolved and not steady state. A theory close to meeting these requirements was published by Penzkofer and Falkenstein. (90) In their paper, an investigation is made of ASE using a time resolved theory; the steady state assumption is never invoked. The quantity and duration of ASE are calculated as a function of various dye and pump parameters; however, no input pulse is included. The theory involves solving 11 coupled first order differential equations numerically.

The output parameters include: fluorescent energy contained in the ASE pulse, duration of the ASE and transmission of the pump pulse. The input parameters which are varied include: pump pulse intensity and duration, dye concentration, solid angle of the active region and the transition cross sections for the various energy levels. Each of the 11 equations describes the evolution in time and space of the population in an energy level or the pump or output light intensity. The cross sections for absorption and the decay rates from each level are required as constants. The dye rhodamine $6 \mathrm{G}$ is used because it is the only dye for which most of these quantities have been tabulated in the literature. 
IV.B.I Initial Conditions

Penzkofer and Falkenstein state the following initial conditions. All of the population is in the ground state, the pump pulse is a gaussian in time with a variable duration and intensity, and there is no initial fluorescence intensity. The ASE is then built up inside the amplifier by spontaneous and stimulated emission. I have altered these conditions by including an input pulse of a gaussian shape and a variable duration, intensity and delay after the pump. The input pulse is then amplified and competes with the ASE for gain. Unfortunately, Penzkofer and Falkenstein give no explanation of how to solve the equations in their paper.

IV.B. 2 Energy Levels

In Figure 4-1, I display the energy level diagram for organic dyes. Levels 1 through 4 are the usual levels for a four level laser as described below. Level 5 is included to add reabsorption. Levels 1 and 4 reside in the ground state which is a singlet $S_{0}$ Levels 2,3 and 5 reside in the first excited state $S_{1}$. Levels 6 and 7 are the states $S_{2}$ and $s_{3}$ respectively. Levels 8 and 9 are the triplet states $\mathrm{T}_{0}$ and $\mathrm{T}_{1}$. Levels 5 through 9 constitute loss mechanisms for the usual four level laser. In the diagram, all of the cross sections are labeled as $\sigma$. The decay rates are labeled by $\tau$ and $K$. My notation conforms to this diagram. 


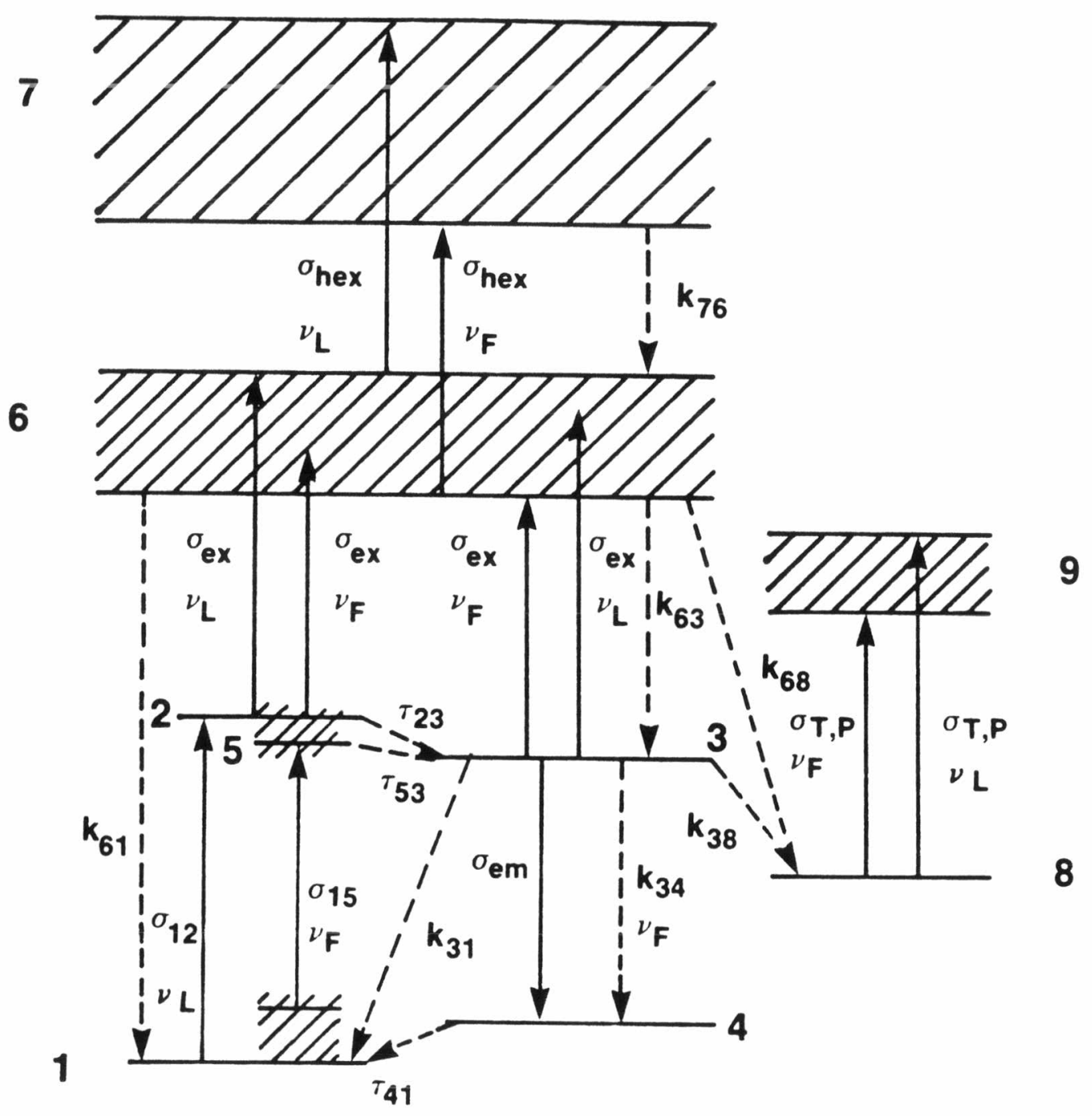

Figure 4-1 Organic dye energy level diagram 
Some of the levels are unimportant and the model can be simplified greatly. The value for $K_{38}$ is $4 \times 10^{5}$ $\sec ^{-1}$ (61); therefore, the $\mathrm{K}_{38}$ rate is quite slow compared to the times of interest. As a result, the populations in levels 8 and 9 never become appreciable and I have neglected these levels. The population in levels 6 and 7 returns largely to level 3. (91) As a result, these levels which have fast relaxation times(92) can be considered as loss mechanisms for both the pump and input pulses. To speed up the calculation, these levels are approximated in most cases. An analysis of the importance of these higher lying excited states is given in Appendix 3. The cross section $\sigma_{15}$ is much smaller than $\sigma_{12}$ and is set to zero both by Penzkofer and myself.

We are now left with the 4 basic laser levels. Before the pump pulse arrives, all the population resides in level 1. By absorbing a photon at the laser frequency $\left(\nu_{L}\right)$ a molecule will be elevated to level 2 but then quickly relaxes to level 3. The lasing transition is between level 3 and 4. The spontaneous emission rate is $\mathrm{K}_{34}$ while the stimulated emission cross section is $\sigma_{\mathrm{em}}{ }^{*}$ From level 4 , the molecule quickly returns to level 1 . The equations that govern the interaction of these four levels with the pump intensity and the fluorescent intensity are given in Table $4-2$. 
POPULATION RATE EQUATIONS

$$
\begin{aligned}
& \frac{\partial \mathbf{N}_{1}}{\partial t}=\sigma_{12} \frac{I_{L}}{\mathbf{h} \nu L}\left(\mathbf{N}_{1}-\mathbf{N}_{2}\right)+\frac{\mathbf{N}_{4}}{\tau_{41}}+\mathbf{K}_{31}\left(\mathbf{N}_{2}+\mathbf{N}_{3}\right) \\
& \frac{\partial \mathbf{N}_{2}}{\partial t}=\sigma_{12} \frac{\mathbf{I}_{L}}{\mathbf{h} \nu_{L}}\left(\mathbf{N}_{1}-\mathbf{N}_{2}\right)-\frac{\mathbf{N}_{2}}{\tau_{23}}-\mathbf{K}_{31} \mathbf{N}_{2} \\
& \frac{\partial \mathbf{N}_{3}}{\partial t}=-\sigma_{\text {em }} \frac{I_{F}}{\mathbf{h} \nu_{F}}\left(\mathbf{N}_{3}-\mathbf{N}_{4}\right)+\frac{\mathbf{N}_{2}}{\tau_{23}}-\left(\mathbf{K}_{31}+\mathbf{K}_{34}\right) \mathbf{N}_{3} \\
& \frac{\partial \mathbf{N}_{4}}{\partial t}=\sigma_{e m} \frac{I_{F}}{\mathbf{h} \nu_{F}}\left(\mathbf{N}_{3}-\mathbf{N}_{4}\right)+\mathbf{K}_{34} \mathbf{N}_{3}-\frac{\mathbf{N}_{4}}{\tau_{41}}
\end{aligned}
$$

\section{INTENSITY RATE EQUATIONS}

$$
\begin{aligned}
& \frac{\partial \mathbf{I}_{\mathbf{L}}}{\partial \mathbf{x}}+\frac{\eta}{\mathbf{c}_{0}} \frac{\partial \mathbf{I}_{\mathbf{L}}}{\partial \mathrm{t}}=-\mathbf{I}_{\mathbf{L}}\left(\mathbf{N}_{\mathbf{1}}-\mathbf{N}_{\mathbf{2}}\right) \sigma_{12} \\
& \frac{\partial \mathbf{I}_{\mathbf{F}}}{\partial \mathbf{x}}+\frac{\eta}{\mathbf{c}_{0}} \frac{\partial \mathbf{I}_{\mathbf{F}}}{\partial t}=\frac{\mathbf{h}_{\nu_{\mathbf{F}}} \mathbf{K}_{34} \mathbf{N}_{3} \Delta \Omega}{4 \pi}+\mathbf{I}_{\mathbf{F}} \sigma_{\mathbf{e m}}\left(\mathbf{N}_{\mathbf{3}}-\mathbf{N}_{\mathbf{4}}\right)
\end{aligned}
$$

\footnotetext{
Table 4-2 Differential equations for the-time resolved theory
} 
I will now define the quantities used in Table 4-2. The quantity $\partial N_{i} / \partial t$ is the partial derivative of the population density $\mathrm{N}_{i}$ (molecules $/ \mathrm{cm}^{3}$ ) with respect to time where $i=1,2,3$ or 4 . The value of this derivative depends on the intensity of the pump laser, $I_{L}$ (ergs/sec $\mathrm{cm}^{2}$ ), the intensity of the emitted light, $I_{F}$, the population densities themselves, $\mathrm{N}_{1}, \mathrm{~N}_{2}, \mathrm{~N}_{3}, \mathrm{~N}_{4}$ and several dye parameters. The frequency of the pump laser is $\nu_{L}\left(\sec ^{-1}\right)$ while the frequency of the emitted light is $\nu_{F}$. The cross sections $\sigma_{12}\left(\mathrm{~cm}^{2}\right)$ and $\sigma_{\text {em }}$ are the absorption and emission cross sections respectively as shown in Figure 4-1. The figure also shows the decay rates $\mathrm{K}_{31}\left(\mathrm{sec}^{-1}\right), \mathrm{K}_{34}, \tau_{23}$ (sec), and $\tau_{41}$ which have as their subscripts the initial and final levels between which the decay occurs.

The quantity $\partial I_{i} / \partial x$ is the partial derivative of the intensity with respect to distance. The two terms $\partial I_{i} / \partial x$ $+\left(\eta / c_{0}\right) \partial I_{i} / \partial t$ govern the transport of the intensity through the amplifier. In the transport terms, $\eta$ is the index of the dye and $c_{0}$ is the vacuum speed of light. In the final equation the first term on the right hand side represents spontaneous emission and $\Delta \Omega$ is the solid angle over which this emission is amplified. The final term in the last equation is the stimulated emission. The origin of the system is defined so that $t=0$ is at the peak of the pump pulse and $x=0$ at the front face of the dye amplifier. 
IV.B. 3 Assumptions

There are certain assumptions and approximations inherent in the model. The times of interest must all be slower than the dephasing time of the molecules $\left(\mathrm{T}_{2} \approx 100\right.$ fs) (93) for the rate equation approximation to be valid. The rate equations are incoherent equations and will not accurately model any coherent effects that occur before the excited molecules dephase. (94) The broad bandwidth of the ASE is not included; a constant emission cross section is assumed for all frequencies. The anisotropic absorption and emission cross sections due to the alignment of the molecules are also omitted. A homogeneous cross section is certainly a good approximation for intense pump pulses. In the intense pumping case nearly all the ground state molecules are excited and an isotropic distribution is approached. (72) Because of the use of short pumping pulses, the ASE in the backward direction is neglected. For very short dye cells or for transverse pumping, this assumption is clearly in error. Finally, the geometry of the amplifier is simplified. A constant pump intensity is assumed across the spatial profile. The ASE sees a constant gain over a given solid angle. The divergence angle is determined by the pump pulse diameter and either the absorption depth of the pump or the cell length, whichever is shorter. 
IV.B. 4 Method of Solution

The first step in solving the set of coupled equations given in Table $4-2$ is to transform the coordinates to a retarded time using:

$$
z=x \quad t=t^{\prime}-\left(\frac{\eta}{c_{0}}\right) x .
$$

Using the chain rule we obtain the following transformations:

$$
\frac{\partial}{\partial t}=\frac{\partial}{\partial t^{\prime}} \quad \frac{\partial}{\partial x}+\left(\frac{\eta}{c_{0}}\right) \frac{\partial}{\partial t}=\frac{\partial}{\partial z}
$$

Physically, this means that in the transformed coordinate system, a point in time "surfs" on the peak of the pump pulse throughout the cell. (94) Next a grid of points in space and time are established. The initial conditions specify that all the population is in level 1 at $t$ ime $t=0$ and for all space. In addition the intensity of the pump $\left(I_{p}\right)$ and input $\left(I_{f}\right)$ are given at $x=0$ for all time. Using the initial conditions, the equations can be used to fill in the values for the populations and intensities at all the unknown points in space and time. Finally, the intensities at the output of the amplifier are calculated.

The method of calculating the value at a later time from a known value proceeds as follows:

$$
\frac{\partial N}{\partial t}=f(t)
$$


becomes

$$
\frac{\Delta N}{\Delta t}=f(t)
$$

and

$$
\Delta N=\Delta t \times f(t),
$$

where $\Delta N$ is the change in the population over a time change of $\Delta t$. Certainly $\Delta t$ must be small enough or the solution will diverge. To test convergence, the numerical solution is computed and then recomputed with a grid spacing of $\Delta t / 2$. If the answer remains the same, the solution has converged.

Unfortunately, the simple method outlined above requires a very small value for $\Delta t$ and so the number of calculations required is prohibitive. A more sophisticated method can be used to reduce the number of calculations required. By first predicting, and then correcting the value of $N$ at a given point in time, the accuracy of the calculation can be made correct to second order. Thus a larger $\Delta t$ may be used to obtain the same accuracy. The comparison of these methods is described more fully in reference 96. One drawback of the predictor-corrector method is that many intermediate values must be stored before the corrected values can be calculated. The storage requirements for the method were too large for the Cyber 170 and so a new method was sought. 
IV.B. 5 Cross Method

Recently, a method was developed by Drummond (97) which offers second order accuracy and greatly reduced storage requirements. A staggered grid is set up in time and space as shown in Figure 4-2. Both the population and the intensity equations are used to calculate a new value at a single point. When the value is obtained, the next points on the grid are found by doubling the difference (see Figure 4-2). It can be shown that the cross method is second order accurate and it does not require any memory of intermediate values. There is another advantage to the cross method which is more subtle. Since we complete each calculation as we proceed, it is possible now to change the value of $\Delta t$ during the computation. We proceed as follows: using the cross method, we calculate the values of all quantities in all of space for the initial time. In Figure 4-2 this corresponds to working our way across the botton row of the grid. Then the next row is calculated. The change in value between these rows is indicative of the convergence of the solution. The size of the next time interval, that is the spacing up to the next row, is chosen based on this criterion. Thus, when the pump is present, the time resolution can be quite high while later in the calculation, the resolution can be decreased to save computer time.

Even with these modifications, the computer program is 
STAGGERED GRID

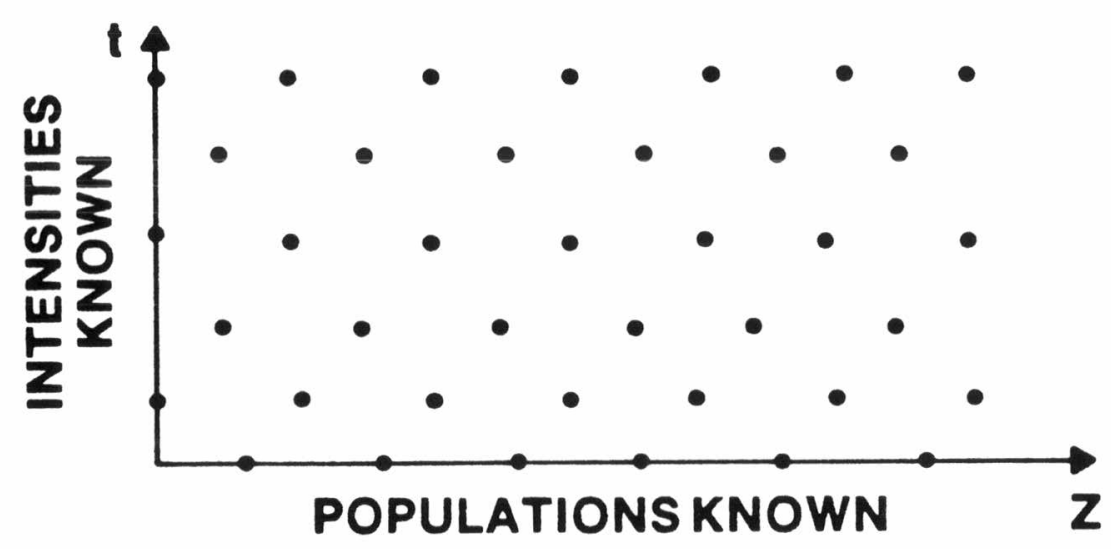

\section{CROSS METHOD}

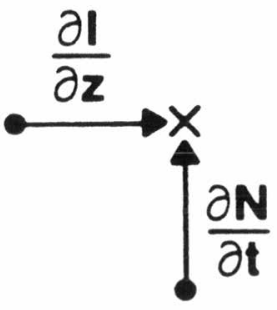

Operate Equations

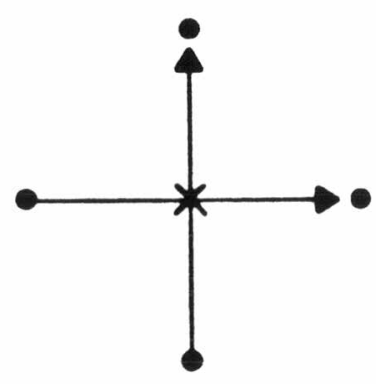

Calculate Next Grid Points

Figure 4-2 Analytical method of solution 
quite lengthy. For a typical calculation with a 70 ps pump pulse, 1000 central processing unit (CPU) seconds are required. If the higher lying excited states are included or a $7 \mathrm{~ns}$ pump pulse is used, the calculation can take as long as $100,000 \mathrm{CPU}$ seconds. The difficulty in solving these equations arises because they are "stiff", that is, the smallest time of interest is $100 \mathrm{fs}$ while the largest can be $5 \mathrm{~ns}$. Since the time resolution must be smaller than the smallest time, a large number of time steps is required. The two dimensional nature also compounds the problem. IV.C Calibration

Certain steps must be taken to calibrate the program: convergence must be tested, reproducibility of previous results demonstrated and the dye parameters adjusted for kiton red. In addition a short cut will be demonstrated for calculating the gain versus delay time curves.

IV.C.I Convergence

An error function is needed to test convergence. I monitored the sum of the population in all of the levels at the end of the calculation. For large $\Delta t$, the sum changed greatly from its starting value. I then plotted the error as a function of $n$. Each time interval was $\Delta t=T / n$ where $T$ is the total time investigated and $n$ is just the number of partitions. Ideally, the error should decrease as a 
quadratic in $n$ because of the second order accuracy. The error, however, was always found to converge more slowly than this. Possible reasons include round-off error in the computer and the error introduced by the space step $\Delta x$. An acceptable error of a few percent was then decided upon and the corresponding size of $\Delta t$ noted. Although $\Delta t$ could be made smaller by the program, the value of $\Delta t$ determined by the above method was set as an upper limit. Finally, the gain was tested and found to change by only a few percent if $\Delta t$ was decreased by a factor of 2 .

\section{IV.C. 2 Previous Results}

One more test of the program was accomplished before it was applied to the experiments. Since the theory was based on that of Penzkofer and Falkenstein ${ }^{(90)}$ it should be able to duplicate their results. The agreement is not perfect because Penzkofer and Falkenstein include anisotropic absorption and treat the higher lying excited states (levels 6 and 7) exactly. In addition, our numerical method of solution is not identical to theirs. (98)

The pump intensity was varied and three quantities were measured for a given concentration. For the case of no input pulse, the total amount of pump energy converted to fluorescent energy $\left(E_{f}\right)$ is tabulated. In addition, the full width half maximum duration of the fluorescent output $\left(\Delta t_{F}\right)$ and the transmission of the pump pulse energy $\left(\mathrm{T}_{E}\right)$ through 
the dye cell were calculated. Penzkofer and Falkenstein's results along with my duplicate results are given in Figure 4-3. The agreement of the theories is quite good over many orders of magnitude.

\section{IV.C. 3 Kiton Red}

The next task was to shift the model from the dye rhodamine $6 \mathrm{G}$ to kiton red. Good values for all the pertinent parameters for rhodamine $6 \mathrm{G}$ (R6G) and rhodamine $B$ (RB) are given by Falkenstein, Penzkofer and Kaiser. (91)

Nowhere in the literature are these values tabulated for kiton red (KR). Drake, Morse, Steppel, and Young (69) have published some information on kiton red. Spectroscopic measurements were also made by this author to verify some results. In addition, when parameters were unobtainable, the values for rhodamine $B$, the most similar dye from the same Xanthene family, were used.

Reference to Figure 4-1 may be valuable during the following section. The absorption cross section for the pump light is $\sigma_{12^{\circ}}$ The peak values for R6G and RB are $4 \times 10^{-16} \mathrm{~cm}^{2}$ but they fall off towards the red as shown in Figure 4-4. (91) Assuming a similar curve for $\mathrm{KR}$ but red shifted by $20 \mathrm{~nm}$ from RB, a cross section of $1 \times 10^{-16} \mathrm{~cm}^{2}$ is obtained at $\lambda=605 \mathrm{~nm}$. Drake's value of $5 \times 10^{-18} \mathrm{~cm}^{2}$ seems unreasonably low. 

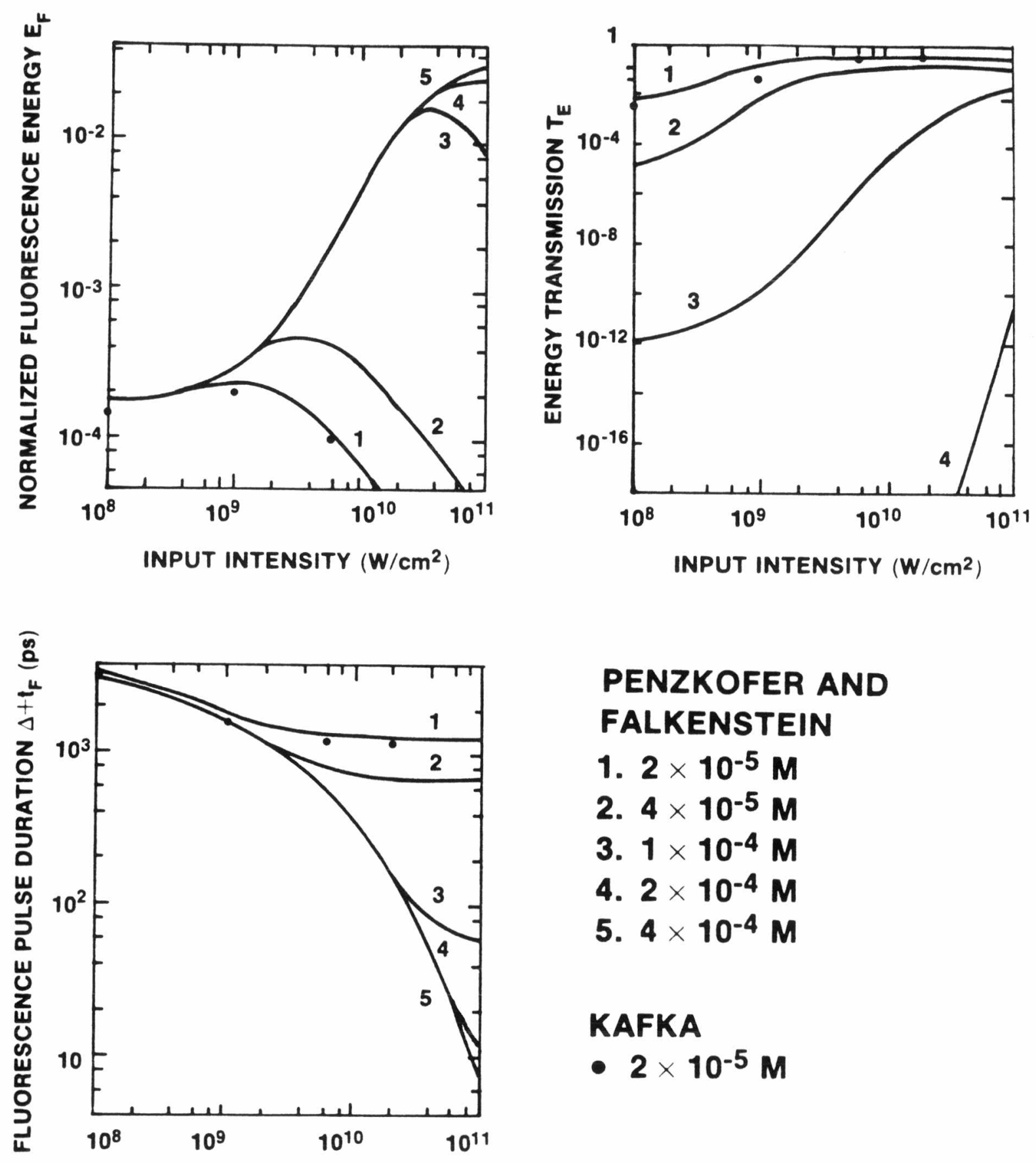

\section{PENZKOFER AND FALKENSTEIN}
1. $2 \times 10^{-5} \mathrm{M}$
2. $4 \times 10^{-5} \mathrm{M}$
3. $1 \times 10^{-4} \mathrm{M}$
4. $2 \times 10^{-4} \mathrm{M}$
5. $4 \times 10^{-4} \mathrm{M}$

\section{KAFKA}

- $2 \times 10^{-5} \mathrm{M}$

INPUT INTENSITY $\left(W / \mathrm{cm}^{2}\right)$

Figure 4-3 Comparison with previous theoretical results 


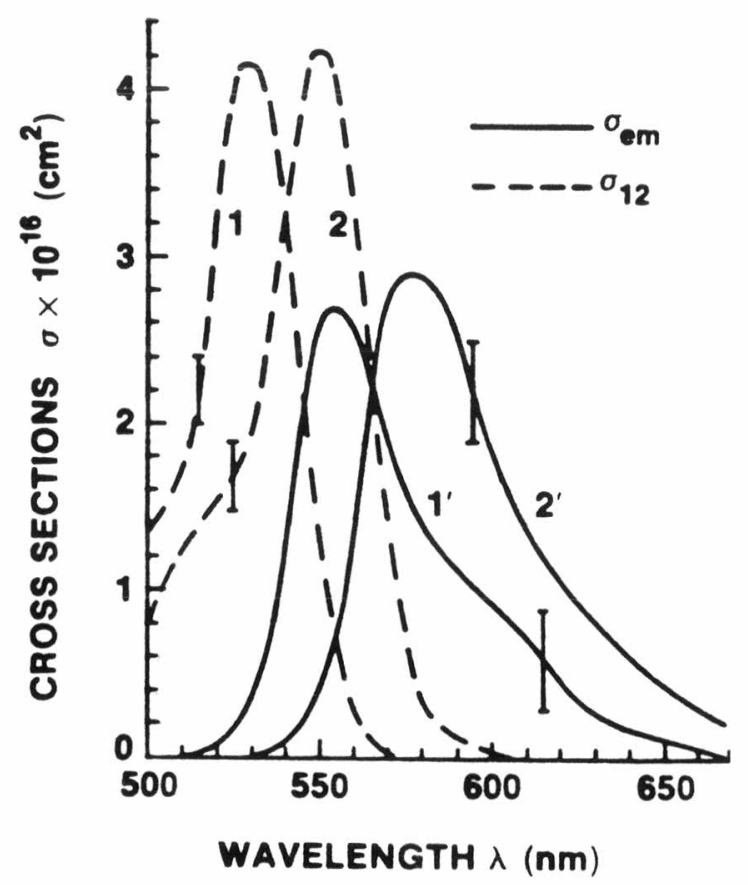

\section{1,1' Rhodamine 6G \\ 2,2' Ahodamine B}

Figure 4-4 Rhodamine absorption and emission spectra 
The values for the higher lying state absorption or relaxation are not in the literature and a measurement is difficult. For this reason the values given for RB are used as the best estimate. Likewise the fast relaxations $\tau_{41}$ and $\tau_{23}$ are assumed similar to RB. All of these are tabulated in Table 4-3. Also included are the values for $\mathrm{h} \nu$ at the pump and input frequencies.

Next we must calculate the spontaneous emission rate. Drake states that triplet states are less important for KR than $R B$ and so we set $K_{38}=0$. This leaves the rates $K_{31}$ and $K_{34}$ to be determined. The fluorescence decay time $T_{f}$ and the quantum yield $Q_{f}$ are given by

$$
\left.T_{f}=\frac{1}{\left[K_{31}+K_{34}\right.}\right]
$$

and

$$
Q_{f}=\frac{K_{34}}{\left[K_{31}+K_{34}\right]} \text {. }
$$

Drake gives values of $\mathrm{T}_{f}=2.8 \times 10^{-9} \mathrm{sec}$ and $Q_{f}=0.83$. Using these, the values of $K_{31}$ and $K_{34}$ are calculated and given in Table 4-3. It should be noted that Falkenstein and Drake give quantum yields of 0.72 and 0.50 respectively for RB.

Since the literature is not consistent, I decided to determine the value for the emission cross section independently. The first step is to measure the fluorescent 


$$
\begin{aligned}
& \sigma_{\text {em }}=3.4 \times 10^{-16} \mathrm{~cm}^{2} \\
& \sigma_{\text {ex }}=6 \times 10^{-17} \mathrm{~cm}^{2} \\
& \sigma_{12}=1 \times 10^{-16} \mathrm{~cm}^{2} \\
& \sigma_{15}=7 \times 10^{-18} \mathrm{~cm}^{2} \\
& h \nu_{L}=3.76 \times 10^{-12} \text { joules } \\
& h \nu_{F}=3.29 \times 10^{-12} \text { joules } \\
& K_{31}=6.11 \times 10^{7} \mathrm{sec}^{-1} \\
& K_{34}=2.96 \times 10^{8} \mathrm{sec}^{-1} \\
& \tau_{41}=1 \times 10^{-12} \mathrm{sec} \\
& \tau_{23}=1 \times 10^{-12} \mathrm{sec}
\end{aligned}
$$


intensity $E(\lambda)$ as a function of wavelength, $\lambda$. The measurement was accomplished with a spectrofluorimeter using $532 \mathrm{~nm}$ excitation of kiton red. Equation 4-8(99) then gives the emission cross section as a function of wavelength.

$$
\sigma_{e m}(\lambda)=\frac{\lambda^{4} K_{34} E(\lambda)}{8 \pi n^{2} c},
$$

where $n=1.33$ the refractive index, $K_{34}=2.96 \times 10^{8} \mathrm{sec}^{-1}$, $c$ is the speed of light and $\lambda$ is the wavelength. The resulting curve is plotted in Figure 4-5 with Drake's curve given for comparison. For $\lambda=605 \mathrm{~nm}$ the value for the emission cross section is $3.4 \times 10^{-16} \mathrm{~cm}^{2}$. To check my method, the above procedure was repeated for R6G. The curve followed that published by Falkenstein (shown in Figure 4-4) to within 10\%. Drake's different result may be attributable to using too concentrated a sample, which could shift the emission spectrum. (31) My sample had a concentration of $2 \times 10^{-6}$ M so that the reabsorption was minimal. 


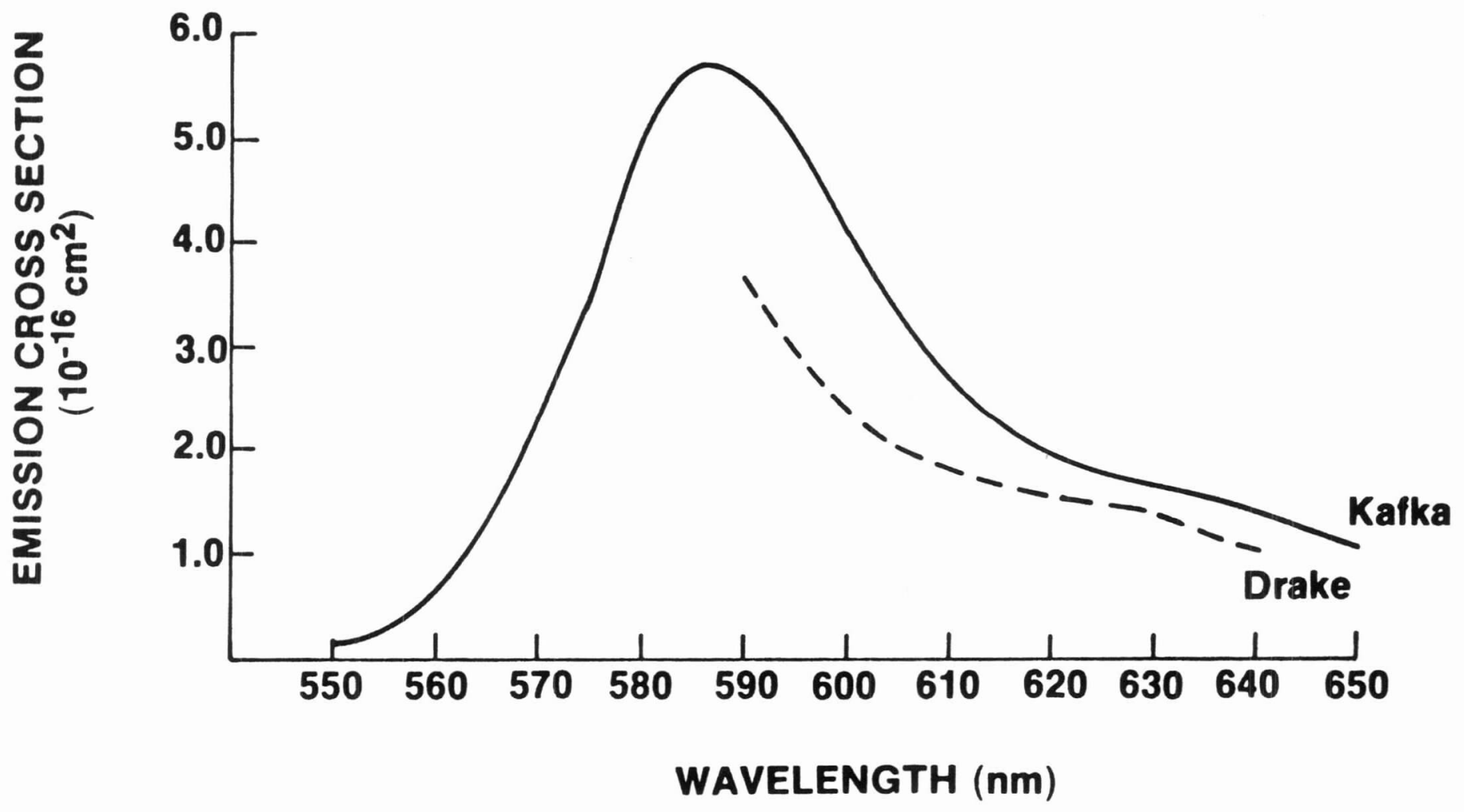

Figure 4-5 Kiton red emission spectra 


\section{IV.D General Amplification Curves}

Now that the theory has been tested and the parameters for kiton red determined, the experiments of Chapter III will be modeled. The central quantity of interest is the time dependent gain in an amplifier. The key input parameters are the pump pulse and input pulse widths and intensities and the time delay between the pump and input pulses. The value of the gain as a function of time will be investigated in section IV.E.

In the present section, I will model the peak gain as a function of the pump and input pulse intensities. The main phenomena of interest is the saturation of the amplifier. Saturation plays a major role in limiting the gain. I have chosen to present the effects of saturation through a family of general amplification curves. The gain is investigated theoretically for a typical case as a function of the pump and input pulse intensities. I have chosen a pump pulse width of $70 \mathrm{ps}$ and input pulse of $2 \mathrm{ps}$. The dye cell is $1.3 \mathrm{~cm}$ long and contains kiton red with a concentration of $2 \times 10^{-4} \mathrm{M}$. The solid angle of $10^{-5}$ is made small to reduce the effects of ASE for now.

IV.D.I Input Intensity

In Figure 4-6 the gain is plotted as a function of input pulse intensity. The procedure is repeated for each 


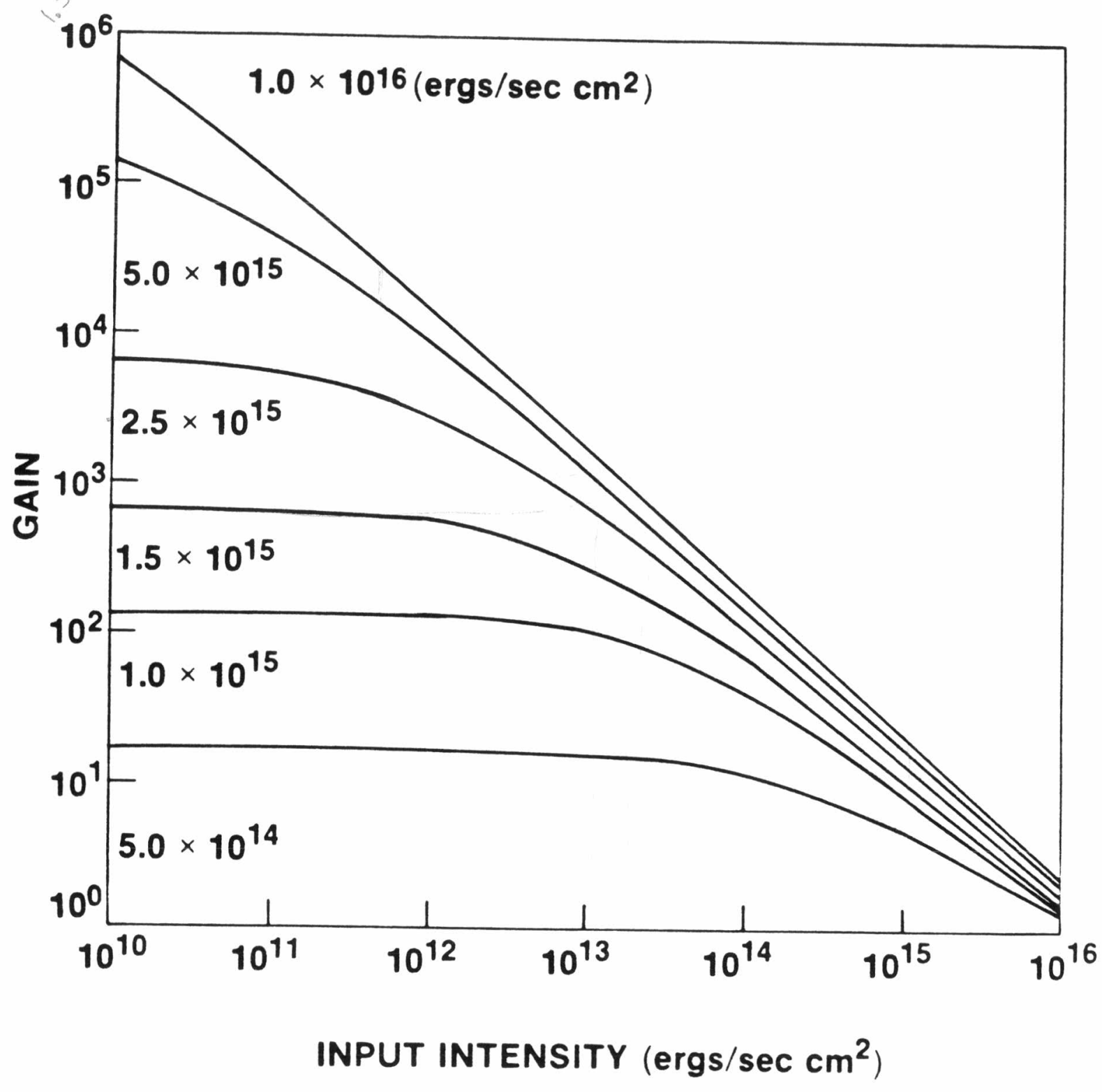

Figure 4-6 Input intensity dependence of the general gain curves 
of six different pump pulse intensities which are labeled on the figure. For any given pump pulse intensity the same general behavior is observed. As the input pulse intensity increases, the gain remains constant for a period and then decreases. The initial gain value is the small signal gain. In the region where the gain is less than the initial value, the amplifier is saturated to some degree. Saturation occurs when half of the excited molecules have been removed by the amplified pulse. The saturation flux is $1.4 \mathrm{~mJ} / \mathrm{cm}^{2}$ which for a 2 ps pulse yields a saturation intensity of $7 \times 10^{15} \mathrm{erg} / \mathrm{sec} \mathrm{cm}^{2}$. The input pulse need only approach the saturation level by the time it exits the amplifier.

Let us examine the two lowest pump intensity curves. The gain of the lowest curve first decreases at an input pulse intensity of $10^{14} \mathrm{erg} / \mathrm{sec} \mathrm{cm}^{2}$. The gain of the next curve first decreases at $10^{13} \mathrm{erg} / \mathrm{sec} \mathrm{cm}^{2}$ but since the gain is greater by 10 the output will be comparable. The above observation, that the intensity of the output pulse will determine the extent to which the amplifier is saturated, can be observed in the behavior of any of the curves in Figure 4-6. Using the general amplification curves, the small signal gain may be calculated for a large range of pump pulse intensities. If the input pulse intensity is also specified, then the saturated gain can be determined.

The measurements presented in section III.F.5 for the 
gain of each amplifier stage are now plotted in Figure 4-7. The gain was measured for many values of the input pulse intensity. The data for each stage is labeled with the number of that stage. The data for the three stages have been shifted to yield a better fit. For the first two stages, the input intensity has been multiplied by a factor of 0.15. For the third stage the correction factor is 0.25 . Given this adjustment, the data fits quite well with the family of curves. The saturation behavior, as well as the decrease in pump intensity in the later stages, is demonstrated accurately.

There are several possible reasons for the input pulse intensity discrepancy. Certain effects have been left out of the theory. Molecular reorientation has not been included. It was assumed that the effect was less important in higher gain stages as explained in section III.D.I. In addition, losses due to triplet states and nonlinear effects, which become more important in higher gain amplifiers, have also been neglected. The spatial cross section of the pump and input beams is assumed uniform, while in reality, the beams are actually gaussian. Finally, the absorption and emission cross sections used to model kiton red are only approximate. In particular, if the emission cross section were actually smaller by a factor of 4, the entire family of curves in Figure 4-7 would shift to approximately 4 times higher input pulse intensities. This 


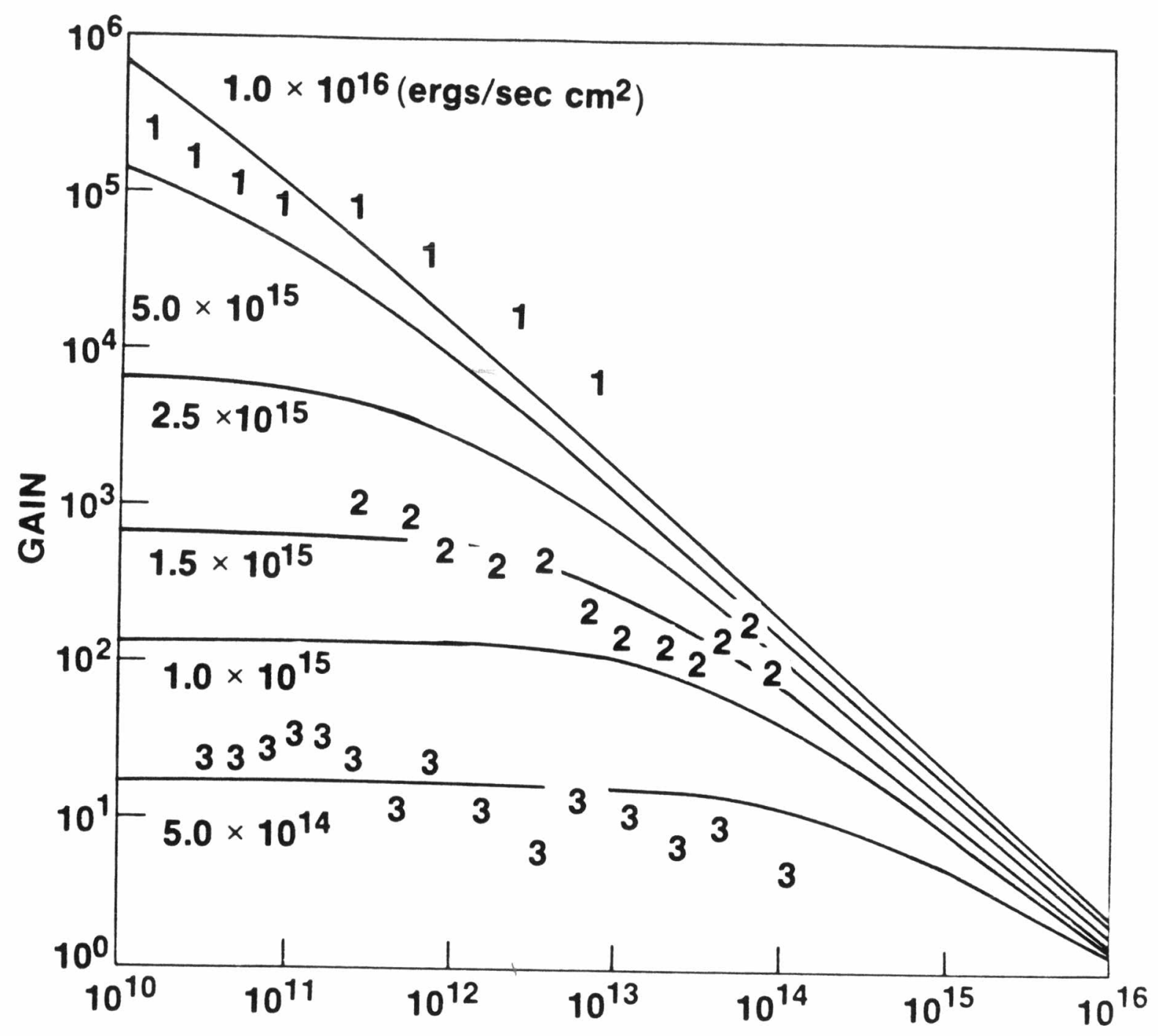

INPUT INTENSITY (ergs/sec $\left.\mathrm{cm}^{2}\right)$

Figure 4-7 Input intensity dependence of the general gain curves with experimental data 
would completely explain the error in the third stage and most of the error in the first two.

It is also possible that the measurements of the data are in error. The input intensity depends on the pulse duration, the incident energy and the spot size. The pulsewidth assumed for the input pulse is either one or two picoseconds. The uncertainty is because the input pulsewidth was measured before and after but not during the experiments. The energy incident on the cell is well characterized but the spot size is not. The spot size changes rapidly in space for the first two stages where a focussing lens is used. The measurements of the spot size are probably accurate within a factor of two; however, the intensity depends on the square of the spot size. If all the uncertainties described above were to add up, the intensity would be in error by a factor of 8 .

A more systematic error could be due to the focussing in the first two stages. I have assumed that all of the incident energy resides within the spot. since the beam quality of the pump and input are not perfect, not all of the energy will focus to a central spot. Some of the energy will be directed into a larger area where it will not contribute to the gain as described in section II.D.7. The spreading effect would lead to a decreased input intensity in any stage where the input is focussed. Focussing is used 
in the first two stages but not the third. A factor of 2 reduction in input intensity for the first two stages is quite reasonable and would account for the remaining discrepancy in the first two stages.

IV.D.2 Pump Intensity

The theory from Figure 4-6 can be recast. In Figure 4-8, the input pulse intensity has been kept fixed and the pump pulse intensity is varied. The initial conditions are the same for the dye cell, dye concentration and pulse durations as in the previous section. For any input pulse intensity, the gain will increase as the pump increases. For small inputs, there is an optimum rate of increase. For larger inputs, the curves diverge from the optimum and approach a limit. The product of the input pulse intensity and the limiting gain is $2 \times 10^{16}$ ergs $/ \mathrm{sec} \mathrm{cm}^{2}$. After this output has been achieved, little more energy can be extracted from the amplifier no matter how hard it is pumped. This is because, for the given concentration, spot size and cell length, there is a fixed number of molecules in the active region and therefore a maximum energy that can be stored.

The data from section III.F.4 is labeled by amplifier stage and plotted with the theory in Figure 4-9. Analogously to the previous section, the pump pulse intensity has been multiplied by a factor to provide a 


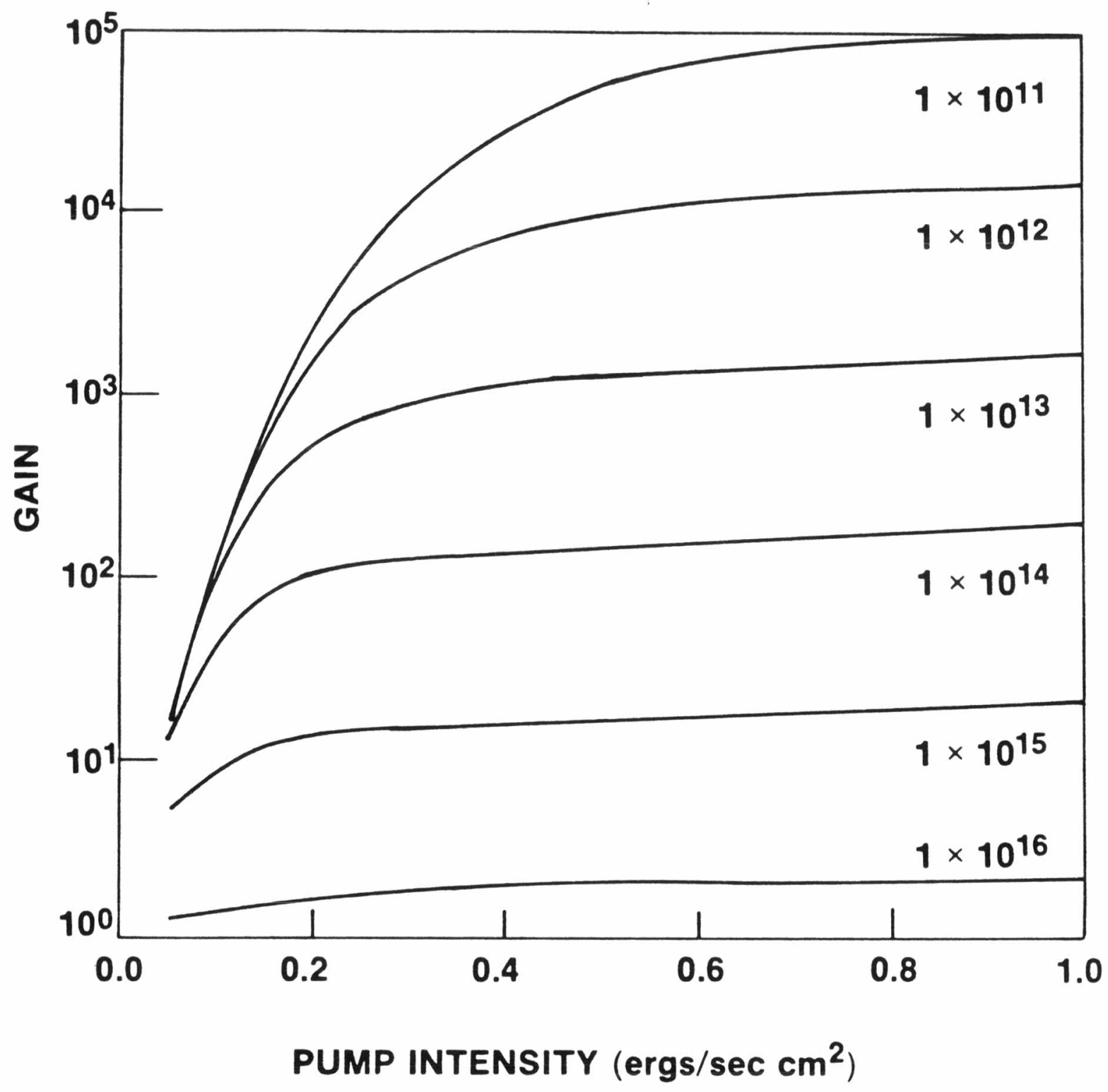

Figure 4-8 Pump intensity dependence of the general gain curves 


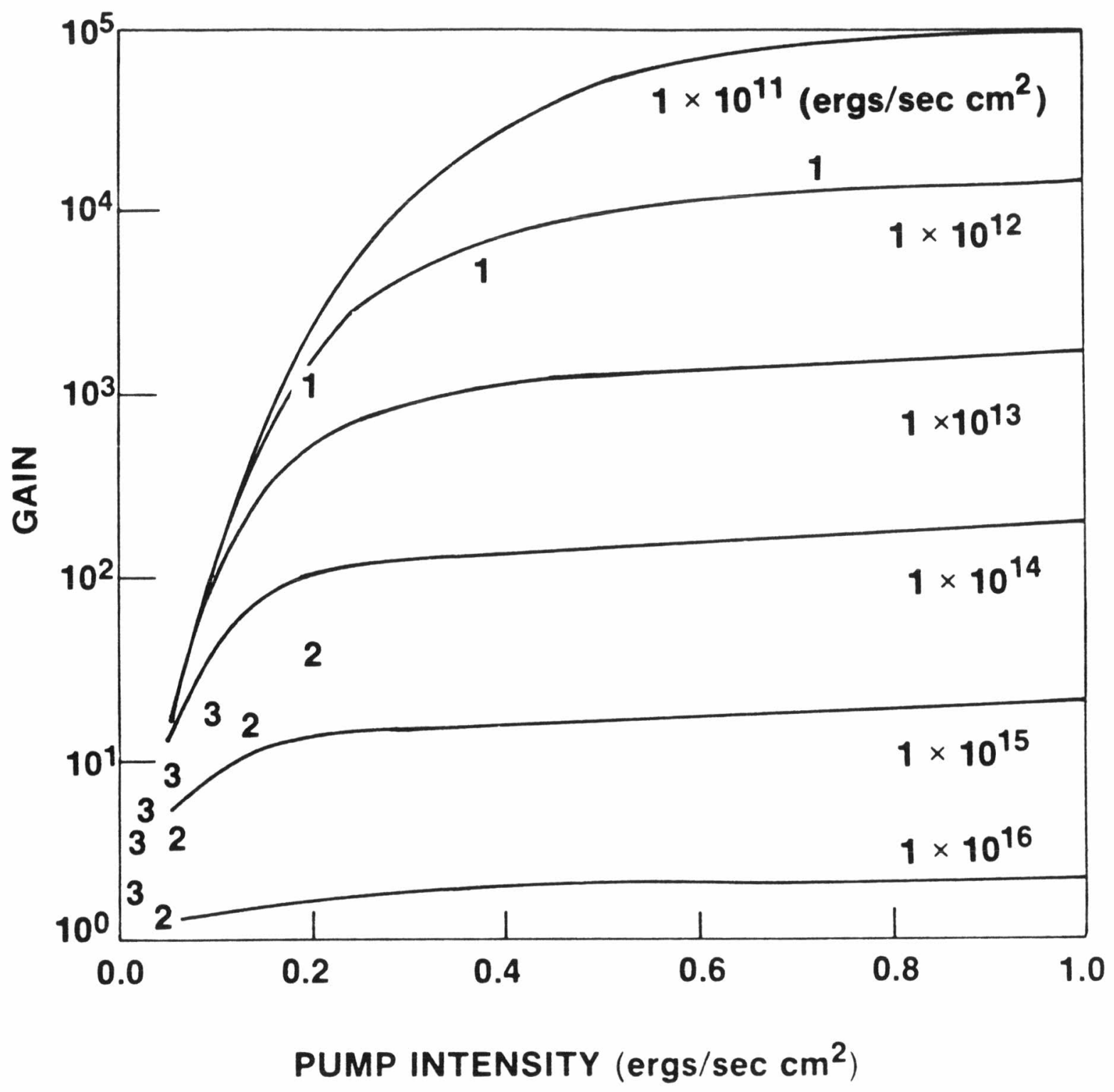

Figure 4-9 Pump intensity dependence of the general gain curves experimental data 
better fit. For the third stage the value is 0.25 and for the first two stages it is 0.15 again. Possible reasons for discrepancies in the experimental data are the same as in the previous section. possible theoretical errors arise from the uncertainty in the value of the absorption cross sections and not the emission cross section as before. While the cross section $\sigma_{12}$ will not play a large role since most of the molecules are already excited, the cross section for excitation to higher lying excited states $\sigma$ ex will. The value of $\boldsymbol{\sigma}$ ex determines the losses that the excited molecules see. If the value for $\sigma$ ex were higher, the curves in Figure 4-8 would shift to higher pump pulse intensity values.

Given the above modifications, the theory models the behavior of the amplifiers. The actual shapes of the experimental data curves fit well with the theoretical family of curves both for the input and the pump series. The saturation of the amplifier is predicted accurately over many orders of magnitude. In addition, the two systems of curves in Figures $4-7$ and 4-9 are self consistent. Recall how the experiments are performed. Each stage was run at its nominal pump and input pulse intensity. Then either the input pulse intensity was decreased by factors of two (Figure 4-7) or the pump pulse intensity was decreased by factors of two (Figure 4-9). The peak input pulse intensity values for the three stages from Figure $4-7$ are $2 \times 10^{12}$, 
$4 \times 10^{14}$, and $5 \times 10^{14} \mathrm{ergs} / \mathrm{sec} \mathrm{cm}^{2}$ after the correction has been made. The same values can be obtained by choosing the best member from the family of curves in Figure 4-9. Likewise, the peak pump pulse intensity values from Figure 4-9 are $1.5 \times 10^{16}, 2 \times 10^{15}$, and $7 \times 10^{14} \mathrm{ergs} / \mathrm{sec} \mathrm{cm}{ }^{2}$ after correction. The same values can be obtained by choosing the best curve in Figure 4-7. Thus, the above modifications lead to a self consistent explanation of the data.

IV.E Timing Curves

In this section, I will duplicate the gain versus time curves given in section III.D. Strictly speaking, to model the experiment a series of computer runs must be accomplished. For each run, the pump and input pulse intensities are fixed but the delay time is varied. The gain is then plotted for each of the delay times and a transient gain curve is built up. Such a procedure requires at least 10 computer runs. However, an approximate method exists which can reduce the number of runs required to one. The method approximates the transient gain with the ASE output from the amplifier and is described below.

IV.E. I ASE Results

My hypothesis is that the quantity of ASE emitted by an amplifier at any time, is a good measure of the gain a small 
signal pulse would see at the same time. Recall from Section III.C.3 that the ASE intensity is a function of both the spontaneous emission at the front of the cell and the small signal gain. The gain depends exponentially on the number density of excited molecules $N(t)$. In a high gain amplifier, a small change in $N(t)$ can cause a large change in gain. The spontaneous emission, however, is only a linear function of $N(t)$. Thus, the noise seeding the amplifier is essentially constant while the gain changes over a large value. It is therefore reasonable to assume that the ASE will track the instantaneous gain quite well in a high gain amplifier.

To test the hypothesis, a series of computer runs were made similar to that pictured in Figure 4-10. The bump $(\approx 1$ to 2 nanoseconds) in the lower portion of the figure is the ASE background for the intense pumping case. The delay time between the pump pulse and the input pulse was then increased and the calculation repeated. The small signal gain was determined for six different values of the delay time. The gain as a function of the delay between the pump pulse and input pulse is shown in Figure 4-11. The data points represent the gain as determined by the method just described. The solid curve is the ASE produced by the pump pulse as a function of time. The ASE curve has been normalized. The shape of the ASE curve duplicates the small signal gain, at the points where it has been calculated, to 


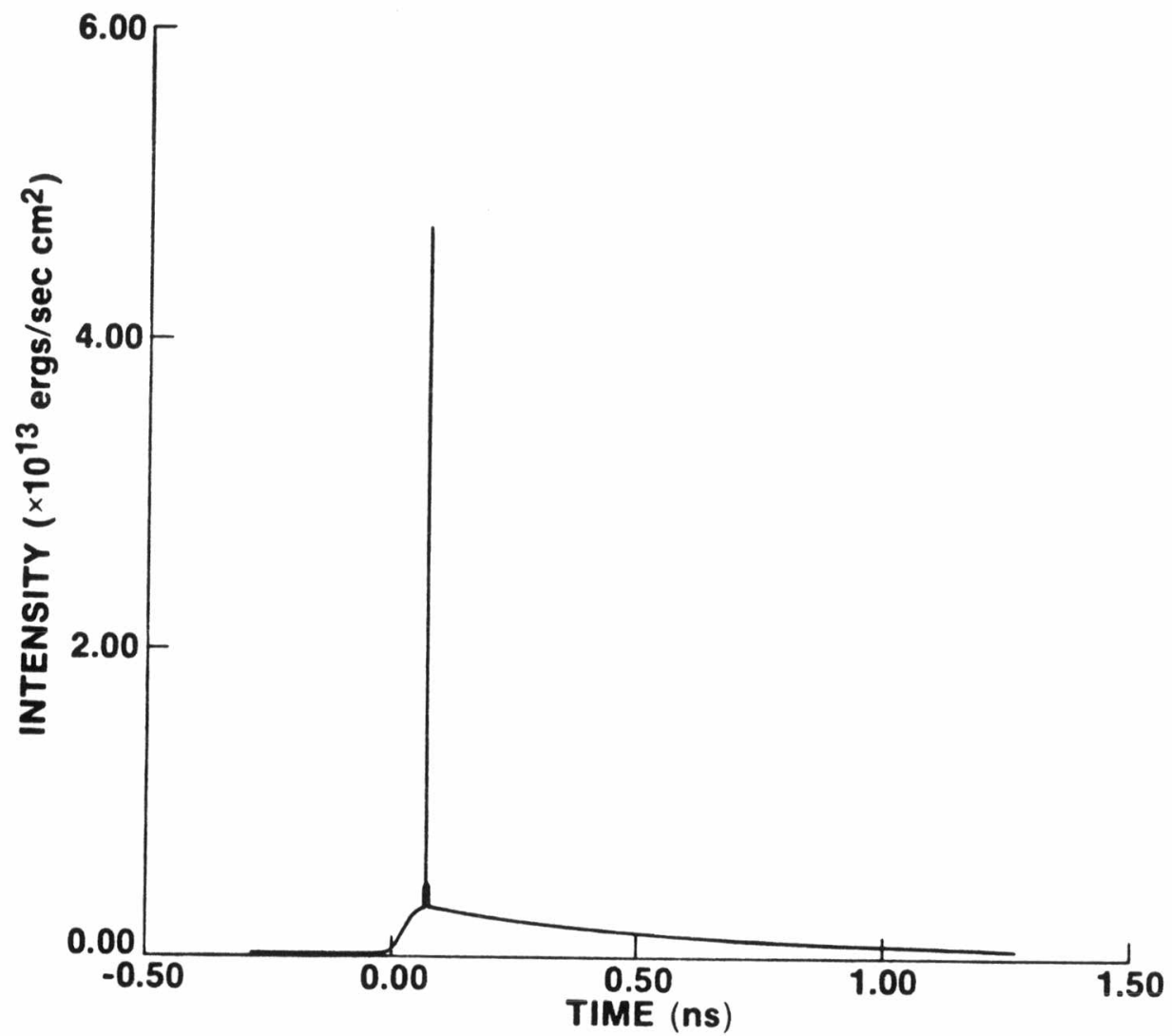

Figure 4-10 Theoretical calculation of the amplified pulse and ASE 


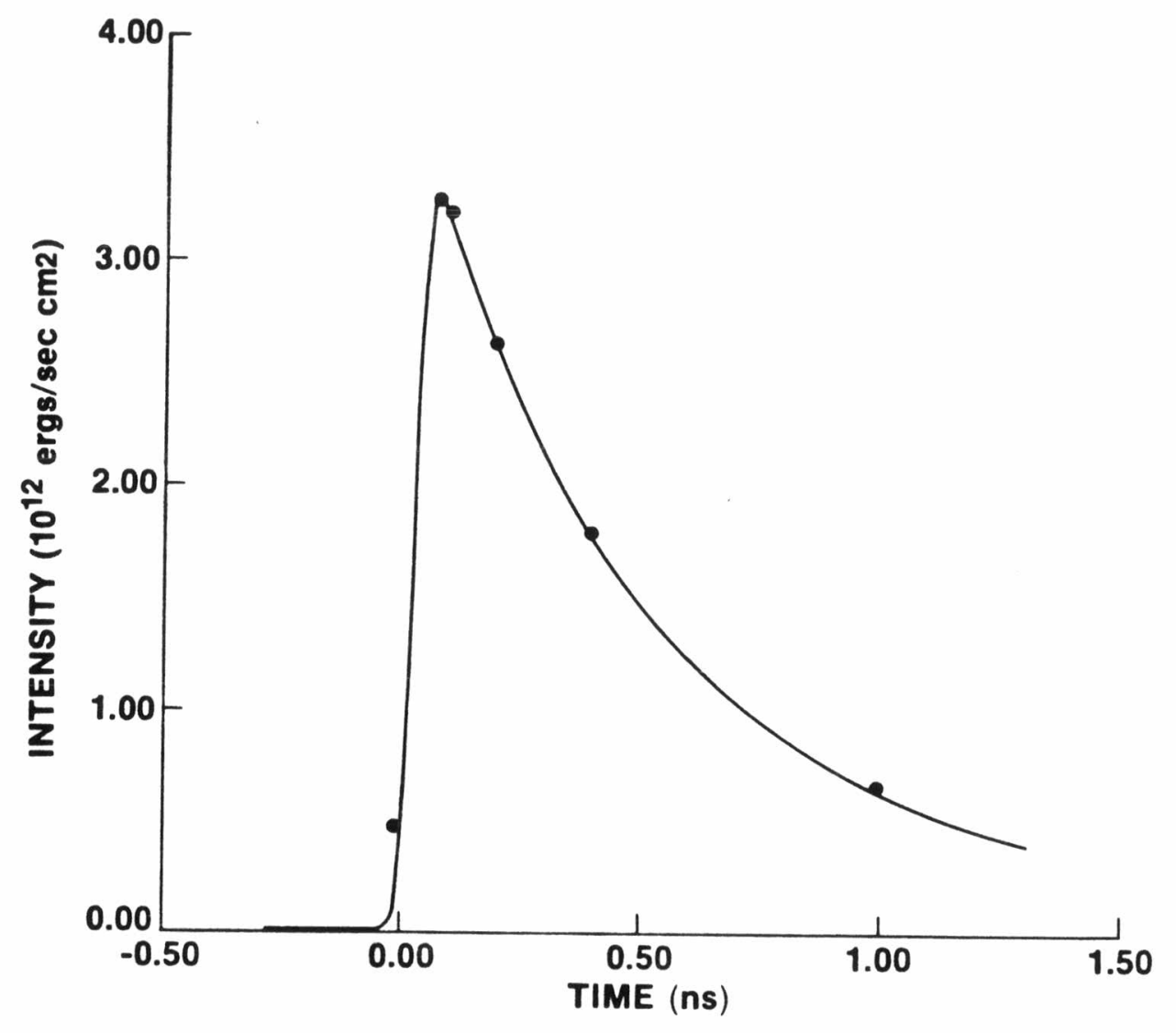

Figure 4-1l Transient gain and ASE 
within 5\%. For this high gain case, the hypothesis outlined above is true; the ASE curve may be substituted for a complete transient gain calculation.

Carrying the implications of this result further, it should be possible to approximate the small signal transient gain of an amplifier experimentally just by observing the ASE produced. A streak camera could be used to perform the measurement; however, the resolution would not be as great as that achieved with the pump-probe technique.

IV.E.2 Comparison with Experiment

One transient gain curve was chosen from the data set used in Figure 3-6. The gain storage time for the curve was $168 \mathrm{ps}$. Since the experiment was conducted in a focussed geometry the experimentally calculated pump intensity was multiplied by a correction factor of 0.10 . In section IV.D, a similar correction factor was determined to give the best theoretical fit. The transient gain was then calculated for a 100 ps pump pulse. The length of the pump pulse affects only the onset of the gain and 100 ps was found to give the best fit. Experimentally, an autocorrelation measurement determined that the pump pulse width ranged from $70 \mathrm{ps}$ to $100 \mathrm{ps}$. In Figure 4-12 the theory is plotted as the solid line and the experimental data as points taken every 100 ps. The two curves have been normalized and the peaks of the two curves have been matched in time. The theory calculates the 


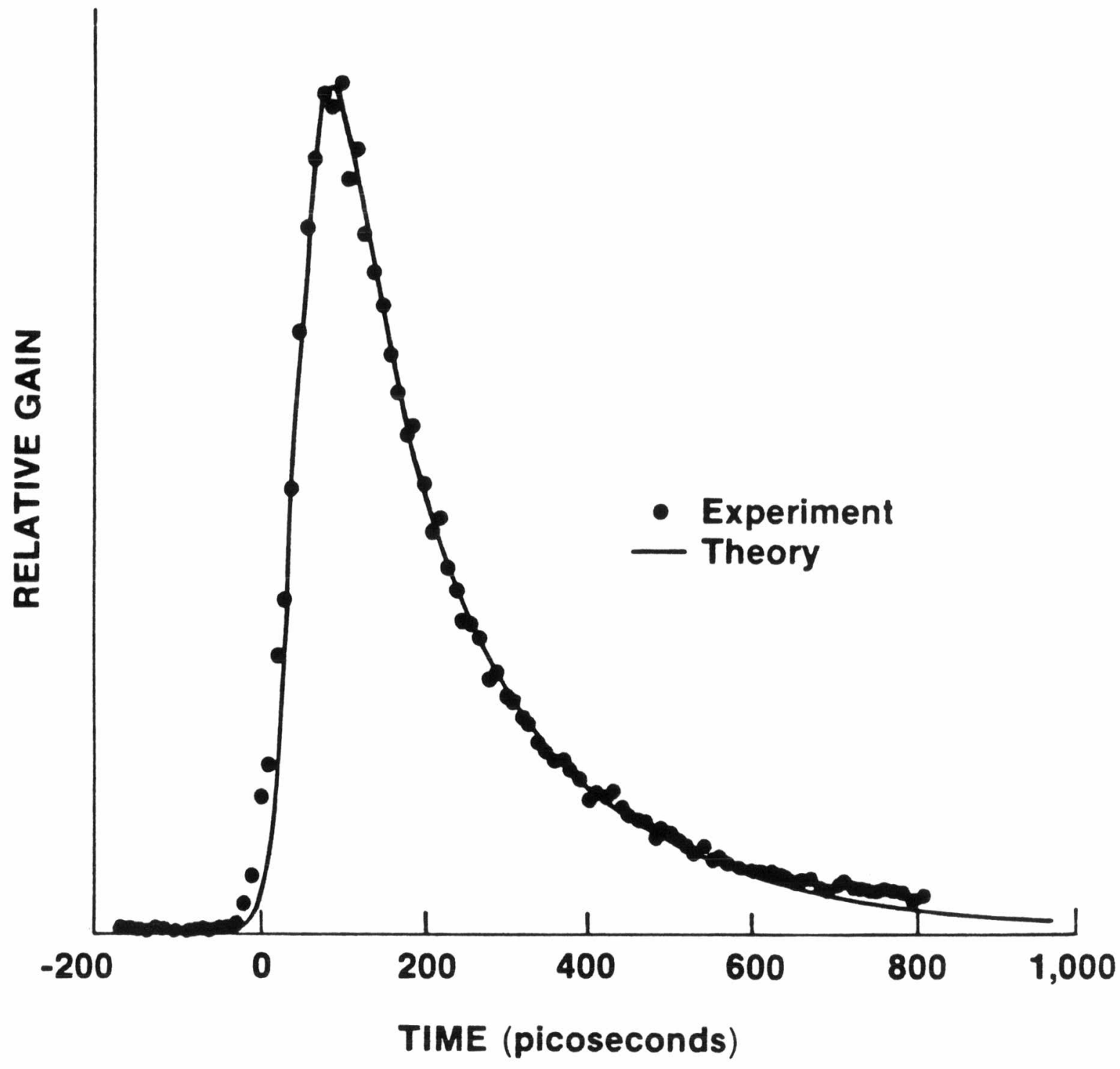

Figure 4-12 Theory and experimental calculation of the transient gain 
gain as a function of delay time between the pump and input pulses and the agreement with the data is remarkable. The slight error in calculating the onset of the gain may be attributed to the assumption that the pump pulse is gaussian.

In conclusion, with a correction factor applied to the pump and input pulse intensities, the theory developed models the experiments of Chapter III to within a few percent. As with previous theories, the saturation of the amplifier is successfully modeled. For the first time, however, the time dependence of the gain is predicted for a picosecond pump pulse. The power of the theory to model the time dependent gain will allow us to compare the efficiency of amplifiers pumped with pulses of picosecond and nanosecond duration.

IV.F Pump Pulse Length

Now that the experiments of Chapter III have been modeled, the theory will be applied to make predictions for experiments which are difficult to perform. One quantity of interest is the system efficiency as a function of the pump pulse duration. As we have stated above, shorter pump pulses give a more efficient system. Comparison of our results with previous work supports this hypothesis (see Section II.A). However, a direct experiment where only the pump pulse duration is changed would be the preferred way to 
test the hypothesis. Experimentally, the pump pulse duration is a difficult quantity to vary. While the pulsewidth of the $C W$ modelocked and Q-switched YAG can be varied from $70 \mathrm{ps}$ to $1 \mathrm{~ns}$ by placing etalons in the YAG cavity, (100) there are additional considerations. The infrared pulse must be frequency doubled and the efficiency of conversion depends on the peak power and thus the pulse duration. As the pump pulse duration changes, the pump pulse bandwidth, divergence, alignment and energy will all change making the experiment practically impossible.

The theory, however, is capable of modeling the system efficiency for varying pump pulse duration. On the surface it appears that all we must do is change one input parameter for the calculation. In practice, the computer program must be adjusted for the long pump pulse case and is slowed down considerably. The results in Figure 4-8 were used as a starting place. For a small signal input $\left(10^{10}\right.$ ergs/sec $\mathrm{cm}^{2}$ ), the gain increases exponentially with increasing pump pulse energy. The exponential relationship yields a straight line when plotted on a semilog graph. In Figure 4-13 we reproduce the gain versus pump energy curve for 70 ps pump pulses, assuming no higher lying states and little $\operatorname{ASE}\left(\Delta \Omega=10^{-5}\right)$. The same gain versus pump energy curve has also been calculated for $35 \mathrm{ps}, 700$ ps and 7 ns pump pulses. 


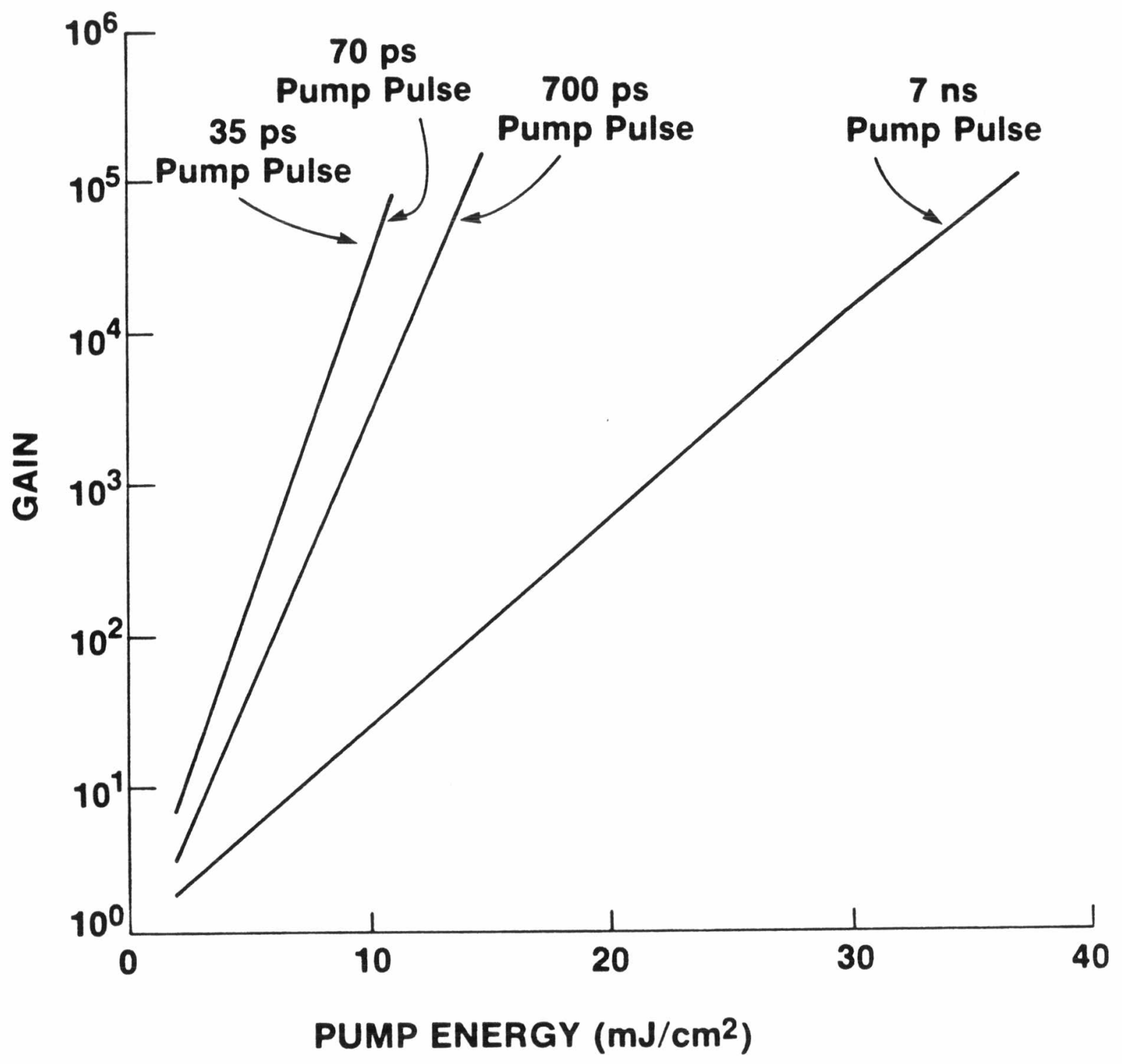

Figure 4-13 Small signal gain dependence on the pump pulse duration 
IV.F.l Simple Model

The first conclusion that can be drawn from Figure 4-13 is that more energy is required in the longer pump pulse to achieve the same gain. The dominant loss for the cases shown in Figure 4-13 is fluorescence since $I$ have intentionally set the solid angle and thus the ASE to a small value. The effect of ASE on these curves will be investigated shortly. Since the curves are nearly linear on the semilog plot, the following hypothesis is proposed. The simple formula for gain versus pump energy still holds but with one modification, given as follows:

$$
G=e^{\sigma N \ell}=e^{\sigma \ell a P}
$$

The gain $G$ is still exponential in pump energy $P$ but a factor $a$ is inserted to account for the losses due to fluorescence during the pump pulse. The efficiency factor $a$ is the fractional number of excited molecules that are created per pump photon. For short pump pulses we expect an efficiency of nearly 1, meaning each photon of the pump pulse creates an excited dye molecule. For longer pump pulses, $\boldsymbol{a}$ will decrease as more pump photons are needed to generate the same number of excited molecules because of the increased losses due to fluorescence. The efficiency factor a can also be viewed as a measure of how much population recycling has taken place before the input pulse arrives. 
The slope of the log gain versus pump energy curve was calculated and the efficiency factor $\boldsymbol{a}$ was tabulated for each of the pulsewidths. The resulting $\boldsymbol{a}^{\prime} \mathrm{s}$ for $35 \mathrm{ps}$, 70 ps, 700 ps and 7 ns excitation are 95\%, 95\%, $75 \%$ and $29 \%$ repectively. This result means that almost all of the molecules excited by the 70 ps pulse are still present when optimum amplification occurs. For the 7 ns pulse, however, more than three times as many pump photons are needed to generate the same number of excited dye molecules because of the fluorescence losses. For pump pulses longer than 7 ns, the efficiency will decrease further. For pump pulses shorter than $70 \mathrm{ps}$, not much change is observed, because essentially all of the energy deposited in the dye is utilized. Fortunately, 70 ps pump pulses are nearly optimum. Longer pump pulses are less efficient. Shorter pump pulses are no more efficent but present a greater synchronization problem.

In conclusion, using modelocked 70 ps pump pulses has been shown theoretically to be 3 or 4 times more efficient than using 7 ns Q-switched pump pulses. The result agrees well with the efficiency numbers of $10 \%$ and $2 \%$ (see section II.A) quoted for the respective high power amplifier systems. 
IV.F.2 Simple Model with ASE

The effects of enlarging the solid angle and hence increasing the amount of ASE can be predicted. (74) At small gains, the ASE is negligible and the curves given in Figure 4-13 will not be altered. At higher gains the addition of ASE will force the gain to lower values. Eventually the ASE will place an upper limit on the gain. These effects are shown in Figure 4-14. The small signal gain is plotted versus pump energy. As the solid angle is increased, the curve rolls over more quickly. Finally for large solid angles the gain becomes limited. Since the ASE depends solely on the gain, the curves will be applicable for any pump pulse duration.

IV.G Pulse Shaping

Pulse shaping effects can also be treated rigorously with the time resolved theory. Any given input pulse shape can be specified either analytically or, with some complication, as data points. In the following example I will use a gaussian input pulse and show what happens when it is amplified in a saturated amplifier. I have chosen the most interesting cases from the general amplification curves which are reproduced in Figure 4-15. The large squares correspond to the cases I have investigated.

I display the input pulse shape and the four normalized 


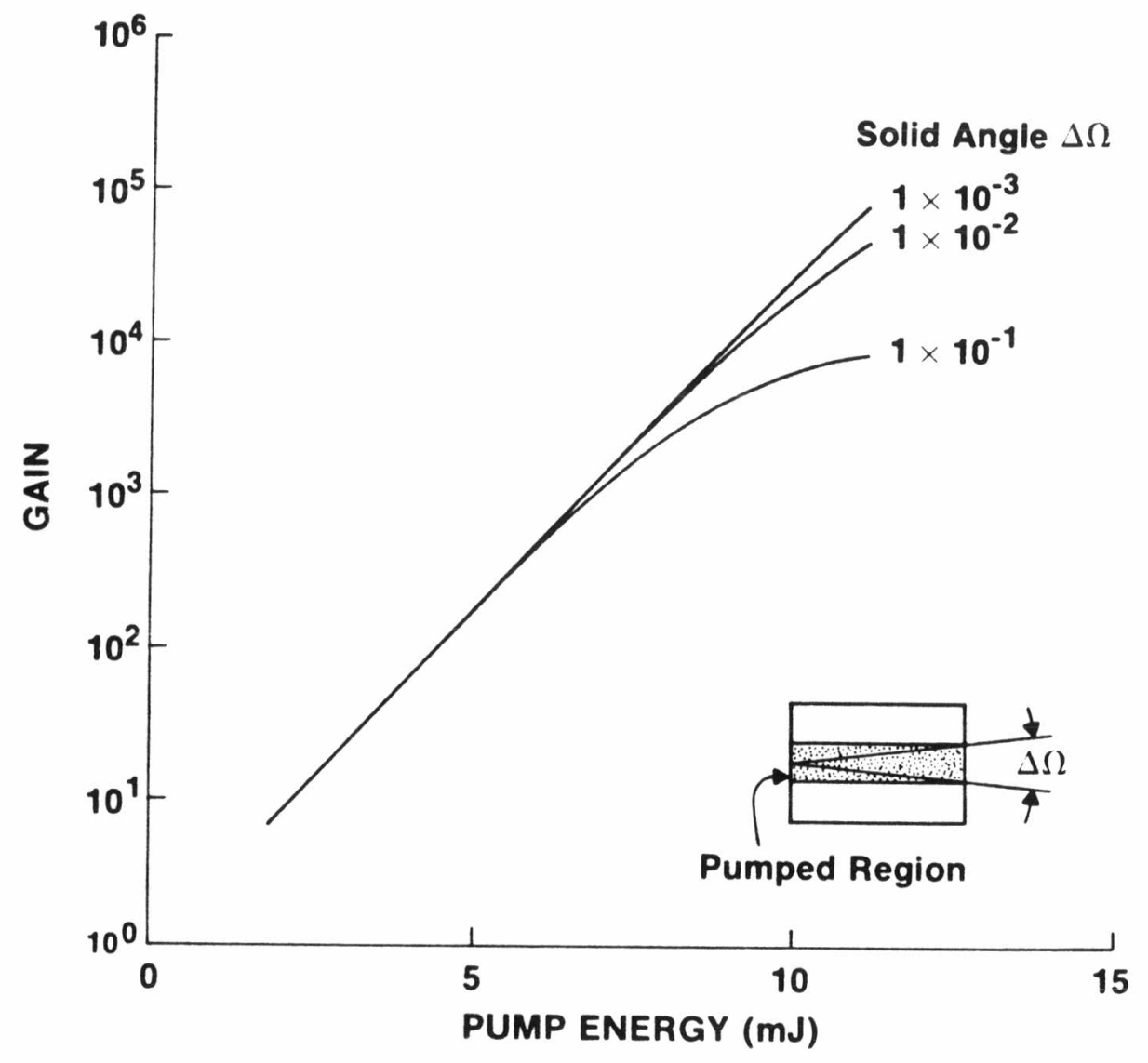

Figure 4-14 Small signal gain dependence on the ASE 


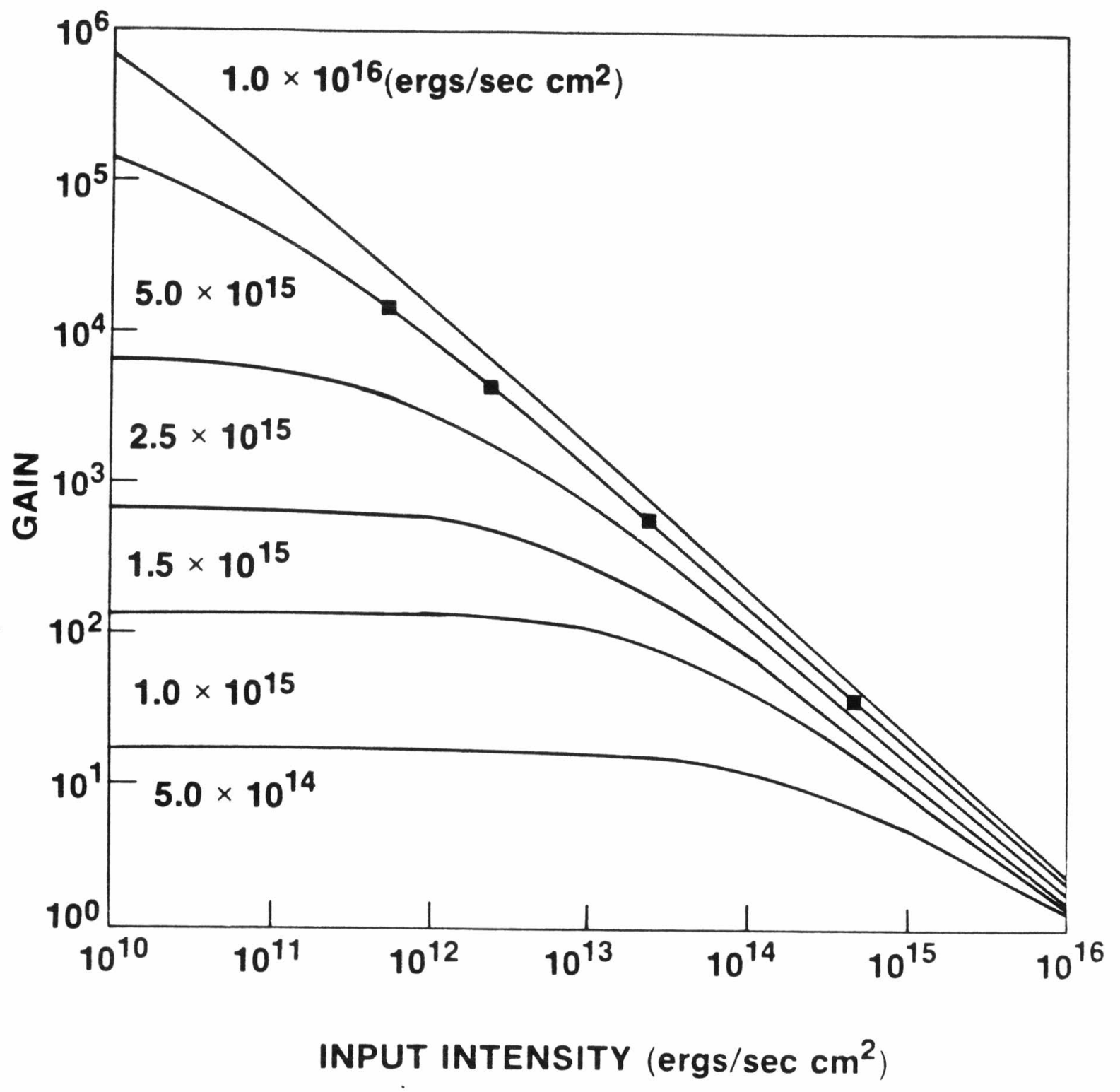

Figure 4-15 Choice of input for the saturated gain 
output pulse shapes in Figure 4-16. The curve furthest to the right is the 2 ps input pulse. The next curve over is the amplified pulse when the input pulse intensity was $10^{12}$ ergs $/ \mathrm{sec} \mathrm{cm}^{2}$. To the left are the amplified pulses for input intensities of $10^{13}, 10^{14}$ and $10^{15} \mathrm{ergs} / \mathrm{sec} \mathrm{cm}^{2}$. In each case, the front edge of the pulse is amplified more than the trailing edge. The preferential amplification of the leading edge occurs because the gain decreases during the pulse duration as the amplifier saturates. There is also a shift in the temporal peak of the output pulse. Since the peak emerges earlier than expected from the amplifier, the pulse has the appearance of traveling faster than the speed of light in the dye.(101) The pulsewidths for each of the output pulses are tabulated in Table 4-4.

The pulse broadening due to saturation depends mostly on the input pulse shape and not its duration. The broadening of a 200 fs gaussian input pulse was also investigated. This case is near the limit of validity for the theory (see section IV.B.3). The same four input energies (each has 10 times the intensity) are used. For either a $200 \mathrm{fs}$ or a 2 ps input pulse of the same energy, the amplified pulse exhibits the same leading edge. The trailing edge of the 2 ps pulse has a slower decay, however, because the gain has a greater chance to recover during the longer pulse. Recall there is still pumping of the amplifier taking place during amplification. For the above 


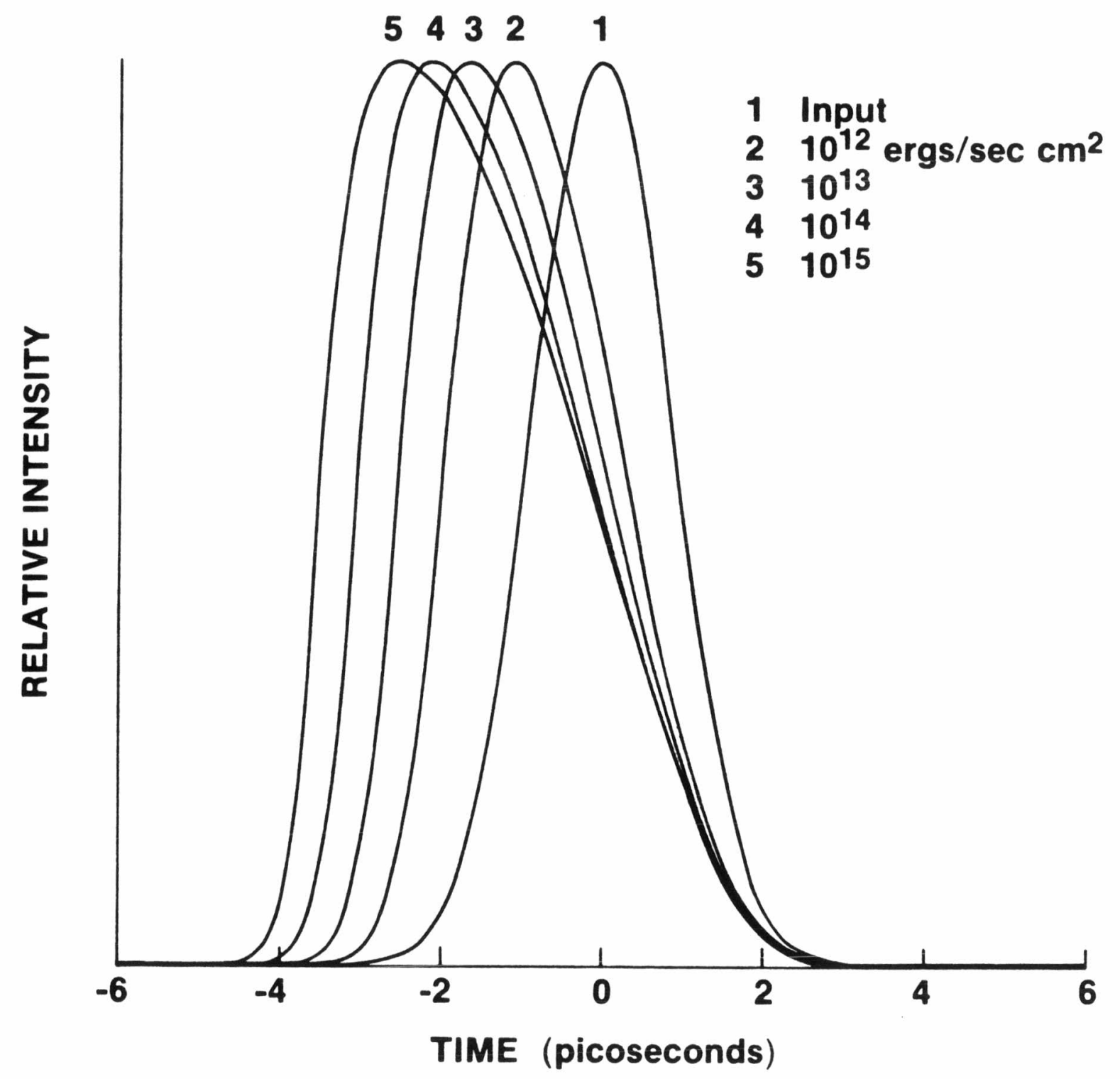

Figure 4-16 Pulse broadening 


\section{INPUT PULSEWIDTH 2 pS}

\begin{tabular}{cc} 
Input Intensity & Pulsewidth \\
\cline { 1 - 2 } $10^{12}$ & 2.38 ps \\
$10^{13}$ & 2.71 \\
$10^{14}$ & 3.07 \\
$10^{15}$ & 3.51
\end{tabular}

\section{INPUT PULSEWIDTH 200 is}

\begin{tabular}{|c|c|}
\hline Input Intensity & Pulsewidth \\
\hline $10^{13}$ & 214 \\
\hline $10^{14}$ & 230 \\
\hline $10^{15}$ & 248 \\
\hline $10^{16}$ & 278 \\
\hline
\end{tabular}

Table 4-4 Pulse broadening due to saturation 
reason, the $200 \mathrm{fs}$ pulse exhibited slightly less broadening as demonstrated by the results in Table 4-4. Other pulse shapes were not investigated but the degree of broadening depends on the leading edge.(102) If the input pulse has a steep rise, the broadening will be less. The relationship between pulse shape and pulse broadening emphasizes the role of the oscillator in producing optimal input pulses for amplification.

IV.H Summary

For the first time, the time dependent value of the gain has been calculated for subpicosecond dye amplifiers. This theory is the only one applicable to the case of pump pulses shorter than the relaxation time of the dye. The time resolved theory includes saturation, fluorescence and ASE losses which limit the gain. A special method of solution was described which speeds up the calculation. Convergence was tested and the parameters for the dye kiton red determined. General amplification curves were presented to illustrate the effects of saturation and comparisons were made with experiment. Theoretical predictions for the transient gain were also presented and matched to experiment.

Finally, predictions were made for experiments that are difficult to perform. Long pulse and short pulse pumping were compared for efficiency and the effects of saturation 
on pulse shape were presented. Future applications might include a detailed analysis of pulse broadening, determination of cross sections and decay rates for other dye systems and, of course, design of larger synchronously pumped dye amplifier systems. 


\section{REFERENCES}

1. A. Penzkofer, A. Laubereau and W. Kaiser, "Stimulated Short-Wave Radiation due to Single-Frequency Resonances of $\mathrm{x}(3), "$ Phys. Rev. Lett. 31, 863 (1973)

2. A. Penzkofer, A. Seilmeier and W. Kaiser, "Parametric Four-Photon Generation of Picosecond Light at $\mathrm{High}$ Conversion Efficiency," Opt. Comm. 14, 363 (1975)

3. R. L. Fork, C. V. Shank, C. Hirlimann, R. Yen and W. J. Tomlinson, "Femtosecond White-Light Continuum Pulses," Opt. Lett. 8,1 (1983)

4. R. S. Adrain, E. G. Arthurs, D. J. Bradley, A. G. Roddie and J. R. Taylor, "Amplification of Picosecond Dye Laser Pulses," Opt. Comm. 12, 140 (1974)

5. A. J. Schmidt, "Generation of a Single Tunable Ultrashort Light Pulse," Opt. Comm. 14, 287 (1975)

6. L. S. Goldberg and C. A. Moore, "Transform-Limited Picosecond Pulse Generation in a Synchronous Modelocked Dye Laser Pumped by Doubled Nd:YAG," Appl. Phys. Lett. 27, 217 (1975)

7. L. S. Goldberg and C. A. Moore, "Synchronous Modelocked Dye Lasers for Picosecond Spectroscopy and Nonlinear Mixing," Laser Spectroscopy 2, (Springer-Verlag, Berlin, Heidelberg, New York, 1975), p. 248

8. D. Huppert and P. M. Rentzepis, "A High-Efficiency Tunable Picosecond Dye Laser," J. Appl. Phys. 49, 543 (1978)

9. Y. Taira and T. Yajima, "High Power Subpicosecond Pulse Generation from a Synchronously Pumped Dye Laser Combined with an External Compressor," Opt. Comm. 29, 115 (1979)

10. L. S. Goldberg, private communication.

11. A. Wokaun, P. F. Liao, R. R. Freeman and R. H. Storz, "High-Energy Picosecond Pulses: Design of a Dye-Laser-Amplifier System," Opt. Lett. 7, 13 (1982)

12. Ultrashort Light Pulses, ed. S. L. Shapiro, (Springer-verlag, Berlin, Heidelberg, New York, 1977), p. 8

13. G. Galilei, "Dialogues Concerning Two New Sciences," ed. H. Crew, A. de Salvio (Northwestern University Press, 
Evanston, Ill, 1950)

14. C. de la Tour, Ann. Chim. Phys. 12, 167 (1819)

15. C. Wheatstone, Phil. Trans. 583 (1834)

16. C. Wheatstone, Phil. Mag. 6, 61 (1835)

17. H. Abraham, and J. Lemoine, Compt. Rend. 129, 206 (1899)

18. H. Abraham, and J. Lemoine, Ann. Chim. 20, 264 (1900)

19. H. W. Moeker and R. J. Collins, Appl. Phys. Lett. 7, 270 (1965)

20. A. J. DeMaria, D. A. Stetser and H. Heynau, Appl. Phys. Lett. 8, 174 (1966)

21. C. V. Shank and E. P. Ippen, "Subpicosecond Kilowatt Pulses from a Mode-Locked CW Dye Laser," Appl. Phys. Lett. 24,373 (1974)

22. Ultrashort Light Pulses, ed. S. L. Shapiro, (Springer-verlag, Berlín, Heidelberg, New York, 1977), p. 88

23. W. Koechner, Solid-State Laser Engineering, (Springer-Verlag, New York, Heidelberg, Berlin, 1976), p. 212

24. W. Koechner, Solid-State Laser Engineering, (Springer-Verlag, $\overline{N e w}$ York, Heidelberg, Berlin, 1976), p. 471

25. Dye Lasers, ed. F. P. Schafer, (Springer-Verlag, Berlin, Heidelberg, New York, 1977), p.168

26. C. V. Shank, R. L. Fork, R. Yen, R. H. Stolen and W. J. Tomlinson, "Compression of Femtosecond Optical Pulses," Appl. Phys. Lett. 40, 761 (1982)

27. Ultrashort Light Pulses, ed. S. L. Shapiro, (Springer-verlag, Berín, Heidelberg, New York, 1977), p. 28

28. Ultrashort Light Pulses, ed. S. L. Shapiro, (Springer-verlag, Berlin, Heidelberg, New York, 1977), p. 90

29. Dye Lasers, ed. F. P. Schafer, (Springer-Verlag, Berlin, Heidelberg, New York, 1977), p. 144

30. Dye Lasers, ed. F. P. Schafer, (Springer-Verlag, Berlin, 
Heidelberg, New York, 1977), p. 167

31. R. F. Kubin and A. N. Fletcher, "Fluorescence Quantum Yields of Some Rhodamine Dyes," Jor. Lumin. 27, 455 (1982)

32. P. Kumar and R. S. Bondurant, "Improving the Pulse Shape in Dye Laser Amplifiers: A New Technique," Appl. Opt. 22,1284 (1983)

33. T. L. Koch, L. C. Chiu and A. Yariv, "Analysis and Performance of a Picosecond Dye Laser Amplifier Chain," J. Appl. Phys. 53, 6047 (1982)

34. T. Sizer II, J. D. Kafka, I. N. Duling III, C. W. Gabel and G. A. Mourou, "Synchronous Amplification of Subpicosecond Pulses," IEEE J. Quantum Electron. QE-19, 506 (1983)

35. A. Migus, C. V. Shank, E. P. Ippen and R. L. Fork, "Amplification of Subpicosecond Optical Pulses: Theory and Experiment," IEEE J. Quantum Electron. QE-18, 101 (1982)

36. R. L. Fork, C. V. Shank and R. T. Yen, "Amplification of 70-fs Optical Pulses to Gigawatt Powers," Appl. Phys. Lett. 41, 223 (1982)

37. Private communications.

38. G. A. Mourou and T. Sizer II, "Generation of Pulses Shorter than $70 \mathrm{fs}$ with a synchronously-Pumped CW Dye Laser," Opt. Comm. 41, 47 (1982)

39. H. Kogelnik, E. P. Ippen, A. Dienes and C. V. Shank, "Astigmatically Compensated Cavities for CW Dye Lasers," IEEE J. Quantum Electron. QE-8, 373 (1972)

40. W. Koechner, Solid-State Laser Engineering, (Springer-Ver lag, New Yor , Hei delberg, Berlin, 1976), p. 511

41. Ultrashort Light Pulses, ed. S. L. Shapiro, (Springer-verlag, Berlin, Heidelberg, New York, 1977), p. 57

42. C. P. Ausschnitt, R. K. Jain and J. P. Heritage, "Cavity Length Detuning Characteristics of the Synchronously Mode-Locked CW Dye Laser," IEEE J. Quantum Electron. QE-15, 912 (1979)

43. K. L. Sala, G. A. Kenney-Wallace and G. E. Hall, "CW Autocorelation Measurements of Picosecond Laser Pulses," 
IEEE J. Quantum Electron. QE-16, 990 (1980)

44. Ultrashort Light Pulses, ed. S. L. Shapiro, (Springer-verlag, Berlin, Heidelberg, New York, 1977), p. 62

45. Dye Lasers, ed. F. P. Schafer, (Springer-Verlag, Berlin, Heidelberg, New York, 1977), p. 142

46. W. Koechner, Solid-State Laser Engineering, (Springer-Ver lag, New York, Hei delberg, Berlin, 1976), p. 447

47. J. M. Forsyth, "Electro-Optics," in Light Modulation (notes for Contemporary Optics, The Institute of Optics, The University of Rochester), p. 19

48. C. Joshi and P. B. Corkum, "Generation of Gigawatt Subnanosecond $1.06 \mu \mathrm{m}$ Pulses by Regenerative

Amplification in ND:Glass," Opt. Comm. 36, 82 (1981)

49. W. Koechner, Solid-State Laser Engineering, (Springer-Ver lag, New York, Heidelberg, Berlin, 1976), p. 397

50. O. Svelto, Principles of Lasers, (Plenum Press, New York and London, 1976), p. 118

51. W. Koechner, Solid-State Laser Engineering, (Springer-Ver $\overline{l a g, ~ N e w ~ Y o r k, ~ H e i ~ d e l b e r g, ~ B e r l i n, ~ 1976), ~}$ p. 598

52. J. P. Campbell and L. G. Deshazer, "Near Fields of Truncated-Gaussian Apertures," J. Opt. Soc. Am. 59, 1427 (1969)

53. W. Koechner, Solid-State Laser Engineering, (Springer-Ver lag, New York, Heidelberg, Berlin, 1976), p. 512

54. W. Koechner, Solid-State Laser Engineering, (Springer-Ver lag, New York, Heidelberg, Berlin, 1976), p. 500

55. The calculation was performed by $R$. Stephen Craxton.

56. The system was constructed by I. N. Duling.

57. G. T. Harvey, C. W. Gabel and G. Mourou, "Synchronization of a Mode-Locked Nd:YAG-Argon Ion Laser system," Opt. Comm. 36, 213 (1981)

58. D. J. Kuizenga, "Short-Pulse Oscillator Developement for 
the Nd:Glass Laser-Fusion Systems," IEEE J. Quantum Electron. QE-17, 1694 (1981)

59. H. Weichel and L. S. Pedrotti, "A Summary of Useful Laser Equations-An LIA Report," EOSD July 1976, p. 22

60. Dye Lasers, ed. F. P. Schafer, (Springer-Verlag, Berlin, Heidelberg, New York, 1977), p. 169

61. D. N. Dempster, T. Morrow and M. F. Quinn, "The Photochemical Characteristics of Rhodamine 6G-Ethanol Solutions," J. Photochem. 2, 343 (1973)

62. Dye Lasers, ed. F. P. Schafer, (Springer-Verlag, Berlin, Heidelberg, New York, 1977), p. 159

63. B. J. Thompson, "Coherent Optical Processing," in Digital and Optical Image Processing, College of Engineer ing and Applied Science, University of Rochester, p. 183

64. J. A. Glaze, "High Enegry Glass Lasers," Proc. SPIE 69 , 45 (1975)

65. W. Koechner, Solid-State Laser Engineering, (Springer-Verlag, New York, Heidelberg, Berlin, 1976), p. 589

66. D. C. Brown, J. M. Rineford, S. D. Jacobs and J. A. Abate, "Electronic, Nuclear and Total Nonlinear Indices of Liquids," Laboratory Report, Laboratory for Laser Energetics, University of Rochester

67. T. Kurobori, Y. Cho and Y. Matsuo, "An Intensity/Phase Autocorrelator for the Use of Ultrashort Optical Pulse Measurements," Opt. Comm. 40,156 (1981)

68. Software was written by Steven zoeller and Tod Sizer II.

69. J. M. Drake, R. I. Morse, R. N. Steppel and D. Young, "Kiton Red $S$ and Rhodamine B. The Spectroscopy and Laser Performance of Red Laser Dyes," Chem. Phys. Lett. 35 , 181 (1975)

70. A. Penzkofer and W. Falkenstein, "Photoinduced Dichroism and Vibronic Relaxation of Rhodamine Dyes," Chem. Phys. Lett. 44,547 (1976)

71. Ultrashort Light Pulses, ed. S. L. Shapiro, (Springer-verlag, Berlin, Heidelberg, New York, 1977), p. 285

72. G. Mourou and M. M. Denariez-Roberge, "Polarization of 
Fluorescence and Bleaching of Dyes in a High-Viscosity Solvent," IEEE J. Quantum Electron. QE-9, 787 (1973)

73. U. Ganiel, A. Hardy, G. Neumann and D. Treves, "Amplified Spontaneous Emission and Signal Amplification in Dye-Laser Systems," IEEE J. Quantum Electron. QE-11, 881 (1975)

74. M. H. Gassman and H. Weber, "Flashlamp-Pumped High Gain Laser Dye Amplifiers," Opto-Elect. 3, 177 (1971)

75. S. A. Rice and G. A. Kenney-Wallace, "Time-Resolved Fluorescence Depolarization Studies of Rotational Relaxation in Viscous Media," Chem. Phys. 47, 161 (1980)

76. H. J. Eichler, V. Klein and D. Langhans, Chem. Phys. Lett. 67,21 (1979)

77. D. Podolski, Ph.D. Thesis, University of Rochester, 1983

78. H. E. Lessing, A. von Jena and M. Reichert, Chem. Phys. Lett. 36,517 (1975)

79. T. J. Chuang and K. B. Eisenthal, Chem. Phys. Lett. Il, 368 (1971)

80. O. Svelto, Principles of Lasers, (Plenum Press, New York and London, 1976 ), p. 263

81. W. Seka, J. Soures, O. Lewis, J. Bunkenburg, D. Brown, S. Jacobs, G. Mourou and J. Zimmerman, "High-Power Phosphate Laser System:Design and Performance Characteristics," Appl. Opt. 18, 409 (1980)

82. W. Seka and J. Hoose, "Short Pulse, Short Wavelength, Optical probe for Laser Plasmas," Laboratory Report, Laboratory for Laser Energetics, University of Rochester, 1983

83. 0. Svelto, Principles of Lasers, (Plenum Press, New York and London, 1976), p. 57

84. J. B. Trenholme and K. R. Manes, "A Simple Approach to Laser Amplifiers," Lawrence Livermore Laboratory Report, 1972

85. A. Yariv, Quantum Electronics, (John Wiley and Sons, New York, Chichester, Brisbane, Toronto, 1975), p. 282

86. L. M. Frantz and J. S. Nodvik, "Theory of Pulse Propagation in a Laser Amplifier," J. Appl. Phys. 34, 2346 (1963) 
87. G. H. C. New, "Mode-Locking of Quasi-Continuous Lasers," Opt. Comm. 6, 188 (1972)

88. G. H. C. New, "Pulse Evolution in Mode-Locked Quasi-Continuous Lasers," IEEE J. Quantum Electron. QE-10, 115 (1974)

89. N. J. Frigo, "Ultrashort Pulse Propagation in Saturable Media: A Simple Physical Model," IEEE J. Quantum Electron. QE-19, 511 (1983)

90. A. Penzkofer and W. Falkenstein, "Theoretical Investigation of Amplified Spontaneous Emission with Picosecond Light Pulses in Dye Solutions," Opt. Quant. Elect. 10, 399 (1978)

91. W. Falkenstein, A. Penzkofer and W. Kasier, "Amplified Spontaneous Emission in Rhodamine Dyes: Generation of Picosecond Light Pulses and Determination of Excited State Absorption and Relaxation," Opt. Comm. 27, 151 (1978)

92. C. V. Shank, E. P. Ippen and O. Teschke, "Sub-picosecond Relaxation of Large Organic Molecules in Solution," Chem. Phys. Lett. 45,291 (1977)

93. G. Mourou, "Spectral Hole Burning in Dye Solutions," IEEE J. QE-11, 1 (1975) and private communication.

94. L. Allen and J. H. Eberly, Optical Resonance and Two-Level Atoms, (John Wiley and Sons, New York, London, Sydney, Toronto, 1975), p. 131

95. J.A. Valdmanis, Ph.D. Thesis, University of Rochester, 1983

96. B. Carnahan, H. A. Luther and J. O. Wilkes, Applied Numerical Methods, (John Wiley and Sons, Inc., New York, London, syåney, Toronto, 1969), p. 341

97. P. D. Drummond, "Central Partial Difference Propagation Algorithms," Com. Phys. Com. 29, 211 (1983)

98. A. Penzkofer, private communication.

99. O. G. Peterson, J. P. Webb. W. C. McColgin and J. H. Eberly, "Organic Dye Laser Threshold," J. Appl. Phys. $\underline{42}, 1917$ (1971)

100. G. F. Albrecht and J. Bunkenburg, "Active-Passive Mode-Locked Oscillator Generating Nanosecond Pulses," Opt. Comm. 38, 377 (1981) 
101. P. G. Kryukov and V. S. Letokhov, Sov. Phys. Usp. 12, 641 (1970)

102. A. Migus, J. L. Martin, R. Astier and A. Orzag, "A Model of Ultra-Short Pulse Amplification," in Picosecond Phenomena II, ed. R. M. Hochstrasser, W. Kasier and C. V. Shank (Springer-Verlag, Berlin, Heidelberg, New York, 1980), p. 59 


\section{APPENDIX 1}

\section{ELECTRONICS}

Although the picosecond synchronization of the pump and input pulses is guaranteed by our design (see section II.C on the optically synchronized system), there are many electronic synchronization problems to be solved. In this appendix, I will describe the electronics necessary to trigger the regenerative amplifier system described in Section II.C.3.

A block diagram of the electronics is displayed in Figure A-1. The clock for the system is a Rockland model 5600 frequency synthesizer which produces a continuous 50 Mhz signal. The signal is split with a Anzac splitter. One part is amplified by an ENI model 320L amplifier to the 10 watt level and then used to drive the modelocker for the CW modelocked YAG laser. The other part is amplified slightly and then fed into a counter. The counter emits two separate signals after a specified number of cycles of the $50 \mathrm{Mhz}$ carrier. The first signal is used to trigger the YAG oscillator and amplifier heads. This signal goes into two pulse generators manufactured by Chronetics (PG-11A) where a specified delay is set. One delayed pulse is a 3 volt fast rise timing pulse and is used to trigger the power supply 


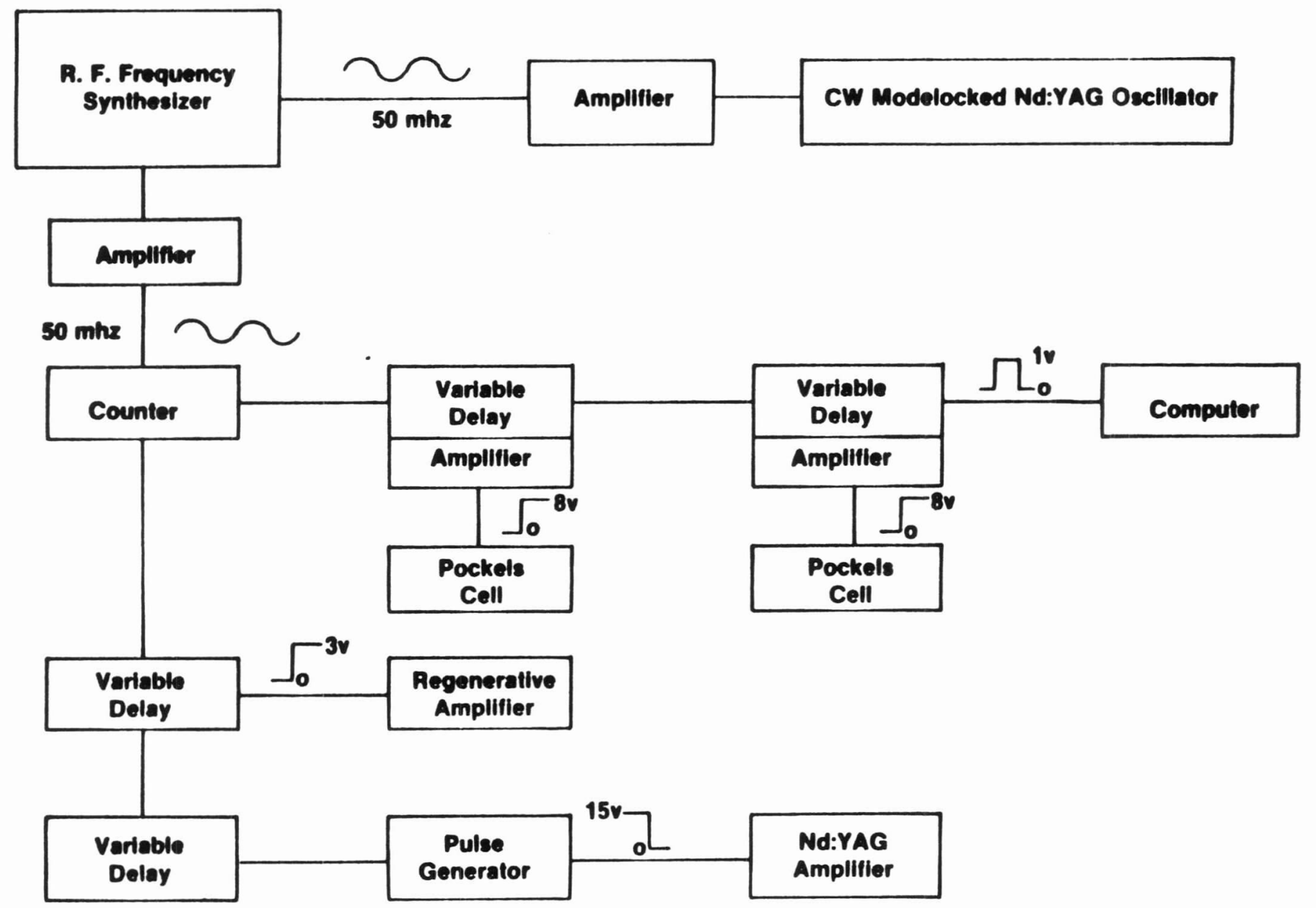

Figure A-1 Experimental setup of the electronics 
for the Raytheon YAG laser head in the regenerative amplifier. The other delayed pulse is sent to a trigger box which prepares a fast falling 15 to zero volt pulse. The electrical pulse triggers the Quantel power supply for the YAG amplifier.

Exactly 400 microseconds after the first pulse has been issued to trigger the flashlamps, the counter box emits its second signal. The second electrical signal proceeds to two variable delay generators manufactored by BNC (Model 7030) . The output of the delay generators is amplified to produce an 8 volt pulse with a nanosecond risetime. These amplified signals are used to trigger the Krytrons which generate the high voltage pulses that switch the Pockels cells. The delay between the Krytrons can be adjusted by one nanosecond increments using the BNC delay generators. In addition, a electrical synchronization pulse is generated to drive the LSI-1I computer during data acquisition.

In summary, the entire system of electronics branches out from a single source, the RF frequency synthesizer. Variable delays, from $1 \mathrm{~ns}$ to $1 \mathrm{~ms}$ are then added to synchronize the required devices. 


\section{APPENDIX 2}

DYE CONCENTRATION

The transient gain was also measured as a function of dye concentration. Kiton red was mixed in $5 \times 10^{-5} \mathrm{M}$, $8 \times 10^{-5} \mathrm{M}$ and $1.5 \times 10^{-4} \mathrm{M}$ solutions. The resultant gain curves are normalized and shown in Figure A-2. Two effects can be seen. First, the fall time of each curve differs with the lower concentration curve having a longer storage time. The longer storage time results, because, at lower concentrations, there are not enough molecules to absorb the entire pump energy. As a result, there are fewer excited molecules in the amplifier and the gain is lower. The lower gain corresponds to the longer storage time as was demonstrated in Figure 3-6.

The second effect is that the risetime differs for each concentration with the rise being delayed for the higher concentration, higher gain case. In our measurement $t=0$ is defined at the front edge of the pump pulse. In Figure A-2 the shift in the rise of the gain is nearly $50 \mathrm{ps}$. There are two causes for the shift. First, the increase in gain itself causes a delay, as explained in section III.B.4, because of the exponential dependence of the gain on the pump. More important, however, is the saturation of the dye 


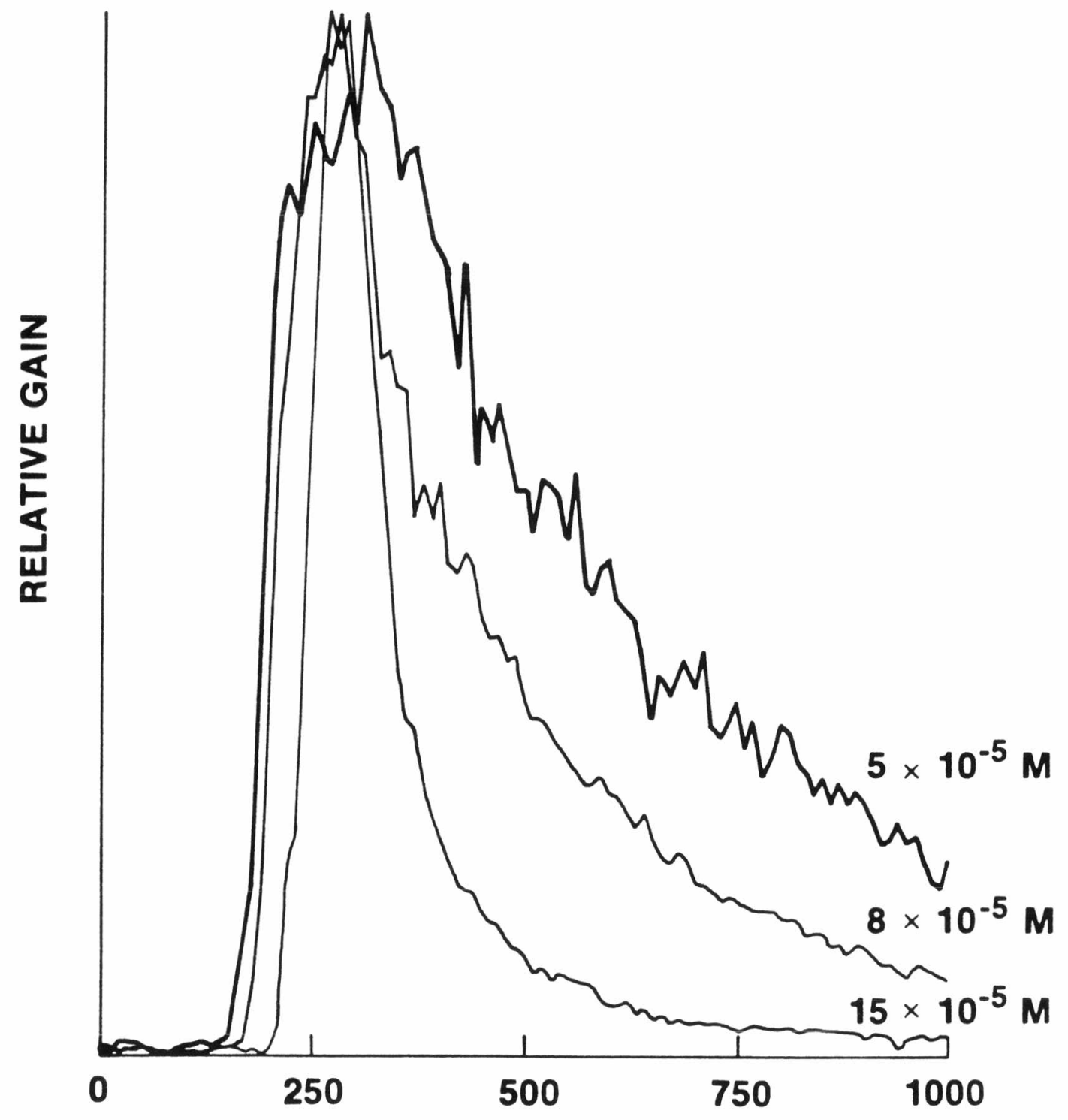

TIME (picoseconds)

Figure A-2 Dye concentration dependence of the gain storage time 
by the pump. For low dye concentration, the front part of the pump pulse creates the gain and excites almost all the dye molecules (bleaching), while the trailing portion just passes through. Thus the gain should be timed with the front of the pump pulse for the low concentration case while the entire pump contributes to the gain in the high concentration case. This interpretation is in strong agreement with the 50 ps delay observed. As was noted previously, the range of concentrations which are of interest is not large. 
APPENDIX 3

HIGHER LYING EXCITED STATES

The four basic energy levels that describe the amplifier were examined in section IV.B.2. In this appendix I will examine the importance of higher lying energy levels. The major level of interest is labeled number 6 in Figure 4-1. The cross section for excitation from level 2 or 3 to level 6 is difficult to measure and not tabulated in the literature for kiton red and so the value for rhodamine B was used. The relaxation time back to levels 2 and 3 is also not known but must be quite fast (see section IV.B.2) . A decay time of $100 \mathrm{fs}$ was assumed ${ }^{(92)}$. Unfortunately, the decay times becomes the fastest rate included in the system of rate equations and thus requires that the resolution of the calculation be made much finer. The result is that including the sixth level explicitly in all calculations becomes prohibitively time consuming.

The importance of the higher lying excited state will not be great when the pump pulse intensity is low. In the low pump regime, the population of level 3 will never become large and the population in level 6 will be even smaller. As the pump pulse intensity increases, however, level 6 becomes a loss mechanism. This is because a second pump 
photon may be required to produce a single excited molecule in level 3. The relaxation time is so fast that the actual population in level 6 is never great. Finally, for very intense pumps, the population in level 6 can become nonzero.

Accordingly, the higher lying states can be treated in three ways. First, they can be totally ignored, as they are by all other authors. Second, they can be put in approximately. In the approximate case, the loss of pump photons is accounted for but the population in level 6 is not. Third, the level can be treated explicity. For the calculations in sections IV.C and IV.F the levels are ignored. In section IV.D where the gain is investigated as a function of pump intensity, and in sections IV.E and IV.G, the level is treated approximately. To conclude this appendix, I will present an exact calculation done in the intense pumping limit.

A 70 ps pump pulse of $10^{18} \mathrm{ergs} / \mathrm{sec} \mathrm{cm}^{2}$ was incident on a thin cell of dye and a quantity of ASE was generated. The pump pulse was intense enough to bleach through the sample with 988 transmission. For the intense pump case, all of the molecules of the dye were excited. Figure A-3 shows the resultant ASE pulse in time for the case of no higher lying excited states. When the excited state, level 6, is added explicitly, the curve changes to that of Figure A-4. Here, there is a temporary hole in the emission. The timing and 


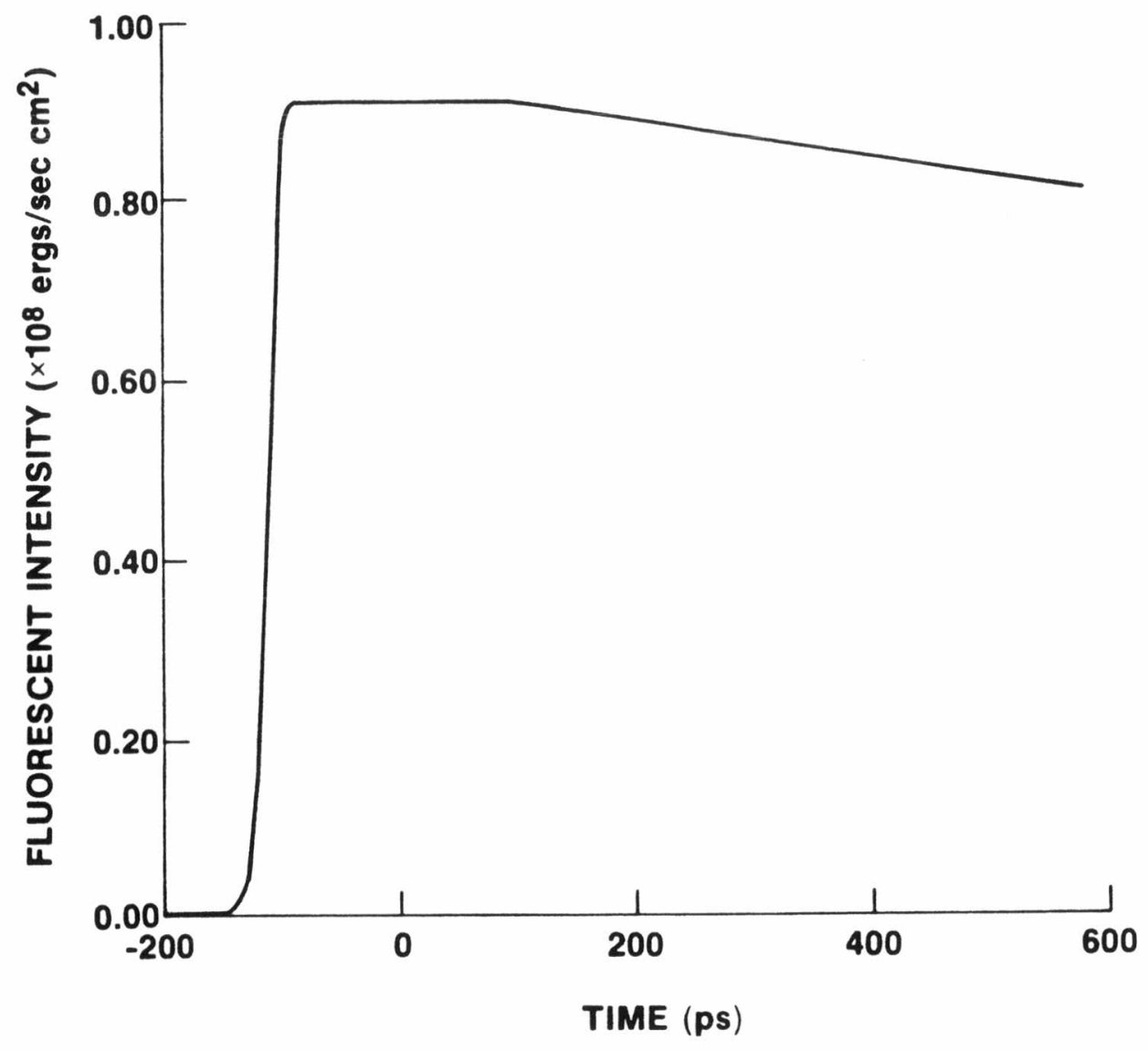

Figure A-3 ASE pulse without the higher lying state absorption 


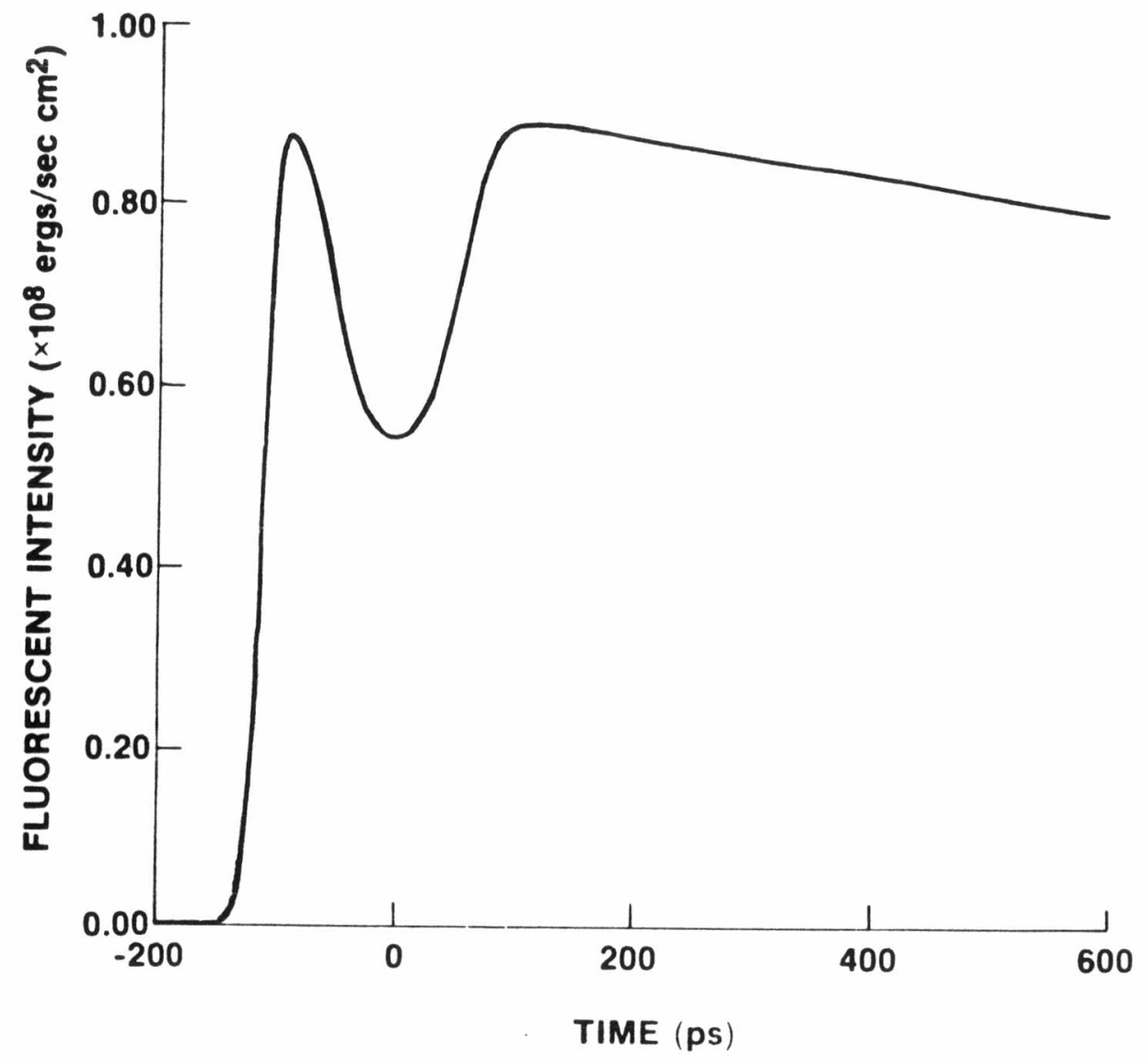

Figure A-4 ASE pulse with the higher lying state absorption 
width of the hole correspond exactly to the 70 ps pump pulse. While the hole is present, up to one third of the molecules reside in the higher lying excited state, 6, while two thirds reside in level 3. For times greater than the pump pulse, the curves in Figures 4-3 and 4-4 are quite similar.

The observation of the hole in the time resolved emission has not been accomplished experimentally but it might be possible to do with a streak camera. If so, we would have a method for determining the cross section for excitation to the higher lying states of organic dyes. 